\title{
Multibody Dynamics Modeling and System Identification for a Quarter-Car Test Rig with McPherson Strut Suspension
}

\author{
Erik Ryan Andersen \\ Thesis submitted to the faculty of the Virginia Polytechnic \\ Institute and State University in partial fulfillment of the requirements for \\ the degree of
}

Master of Science

In

Mechanical Engineering

Dr. Corina Sandu, Chair

Dr. Steve C. Southward

Dr. Mary Kasarda

May 3, 2007

Blacksburg, Virginia

Keywords: Multibody Dynamics, System Identification, Quarter-Car, McPherson Strut 


\title{
Multibody Dynamics Modeling and System Identification for a Quarter-Car Test Rig with McPherson Strut Suspension
}

\author{
Erik Ryan Andersen
}

\begin{abstract}
For controller design, design of experiments, and other dynamic simulation purposes there is a need to be able to predict the dynamic response and joint reaction forces of a quarter-car suspension. This need is addressed by this study through development and system identification of both a linear and a non-linear multibody dynamics McPherson strut quarter-car suspension model.
\end{abstract}

Both models are developed using a method customary to multibody dynamics so that the same numerical integrator can be used to compare their respective performances. This method involves using the Lagrange multiplier form of the constrained equations of motion to assemble a set of differential algebraic equations that characterize each model's dynamic response. The response of these models to a band-limited random tire displacement time array is then simulated using a Hilber-Hughes-Taylor integrator.

The models are constructed to match the dynamic response of a state-of-the-art quartercar test rig that was designed, constructed, and installed at the Institute for Advanced Learning and Research (IALR) for the Performance Engineering Research Lab (PERL). Attached to the experimental quarter-car rig was the front left McPherson strut suspension from a 2004 Porsche 996 Grand American Cup GS Class race car. This quarter-car rig facilitated acquisition of the experimental reference data to which the simulated data is compared. 
After developing these models their optimal parameters are obtained by performing system identification. The performance of both models using their respective optimal parameters is presented and discussed in the context of the basic linearity of the experimental suspension.

Additionally, a method for estimating the loads applied to the experimental quarter-car rig bearings is developed. Finally, conclusions and recommendations for future research and applications are presented. 


\section{ACKNOWLEDGMENTS}

I would like to thank Dr. Corina Sandu for taking the time to work with and guide me through this thesis. Your patience and flexibility in atypical circumstances went above and beyond any known standard. I would also like to thank Dr. Steve Southward for your guidance and encouragement. Your willingness and ability to guide me through technical issues I encountered during this work was incredibly helpful. Also, I would like to thank Brendan Chan for your tutelage and for going beyond the call of duty to help me through this process. Discussing the finer points of multibody dynamics over pizza made if all the more enjoyable. I couldn't have asked for a better team.

I would also like to thank Dr. Mary Kasarda for agreeing to be on my committee and providing excellent feedback. Thanks are also due to Dr. Mehdi Ahmadian for graciously and patiently steering me in the right direction.

To my mom, thank you for teaching and encouraging me to have the serenity to accept the things I cannot change, the courage to change the things I can, and the wisdom to know the difference. To my dad, thank you for providing unyielding stability and peace of mind. Also, thank you for providing a constant address throughout my many moves. To my brother, Peder, thank you for teaching me that we are only bounded by our imagination.

To my grandmother, Elvira Andersen, thank you for exemplifying patience, tenacity, and kindness. Your wisdom enriched all whose lives you touched.

To Victor Goldthorp, Elsie Goldthorp, Peder Andersen, Freda Sutton, Spencer Sutton, Timothy Sutton, Leslie Jackson, and Beth Johnson, thank you all for the support and balance you brought to me through this journey. Your humor and goodwill kept this ship sailing.

I would also like to thank all of my friends in AVDL and PERL for giving these labs personality and for the many laughs we shared. Best of luck to you all. 


\section{CONTENTS}

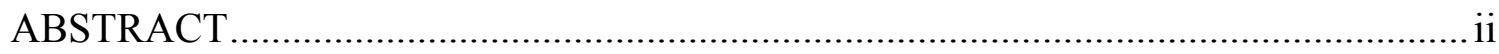

ACKNOWLEDGMENTS .................................................................................. iv

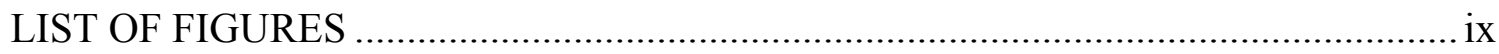

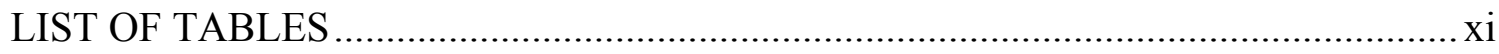

LIST OF SYMBOLS …....................................................................................

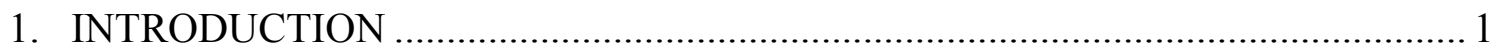

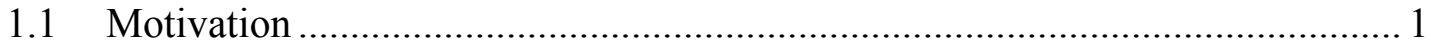

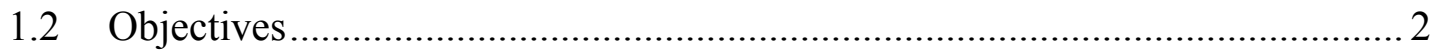

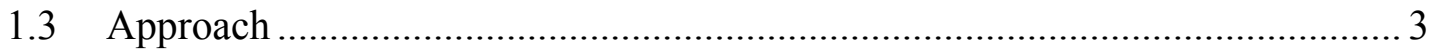

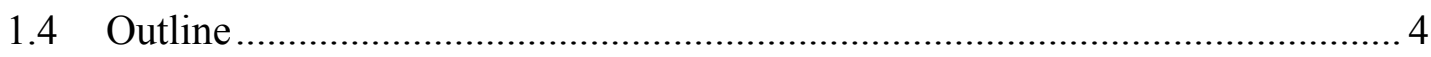

2. LITERATURE SEARCH .................................................................................. 5

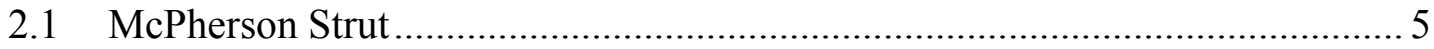

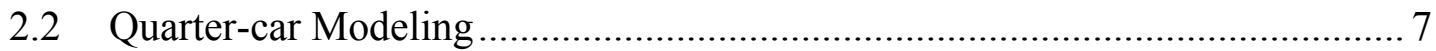

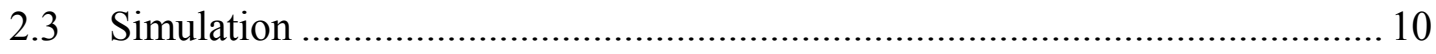

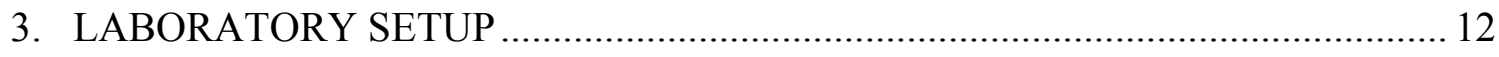

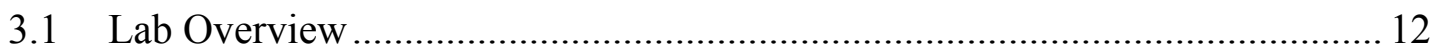

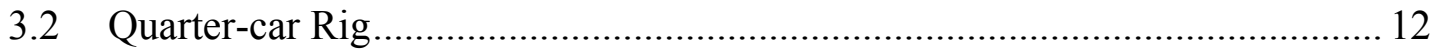

3.3 Hydraulic Driving Equipment ................................................................ 16

3.4 Electronic Driving and Measuring Equipment...................................... 17

3.5 Data Collection Procedure .................................................................... 20

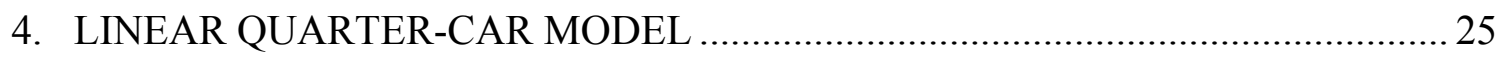

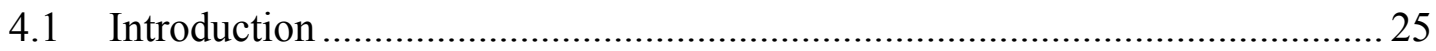

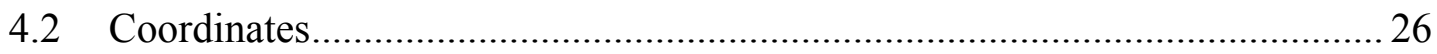

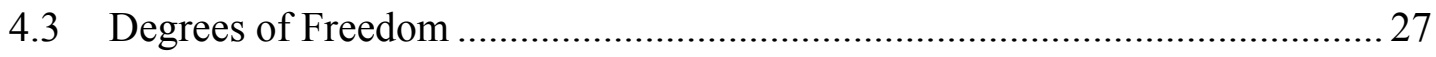

4.4 Kinematic Assumptions and Constraints ............................................. 28

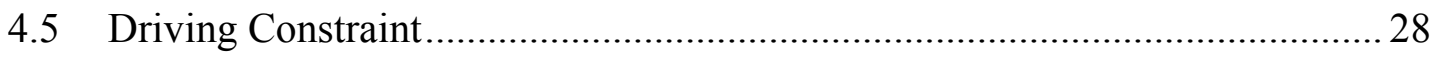




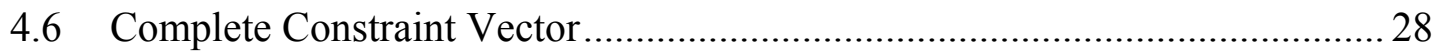

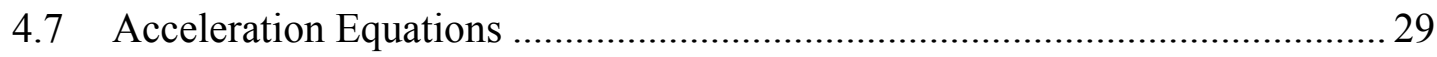

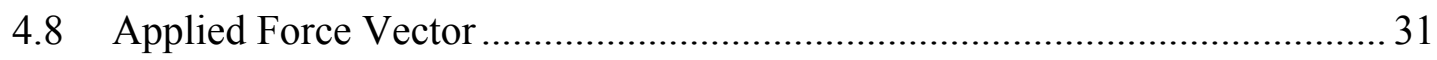

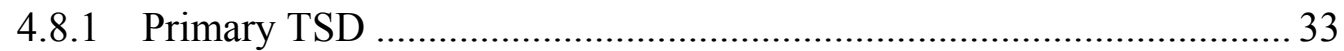

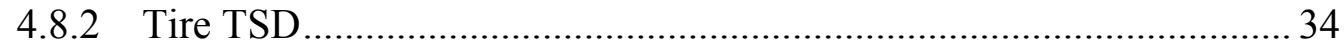

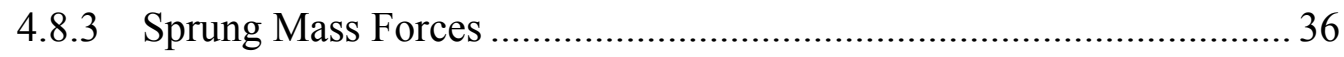

4.8.4 Unsprung Mass Forces ...................................................................... 36

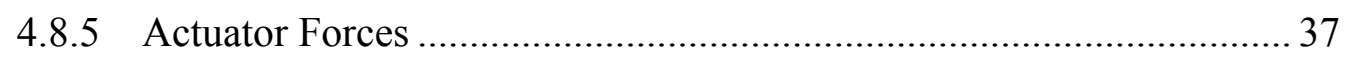

4.8.6 Complete Force Vector ……………………………........................... 37

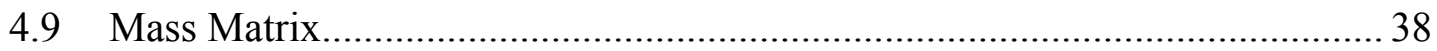

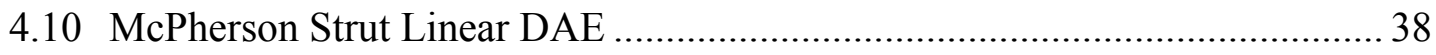

5. LINEAR QUARTER-CAR MODEL SYSTEM IDENTIFICATION ........................39

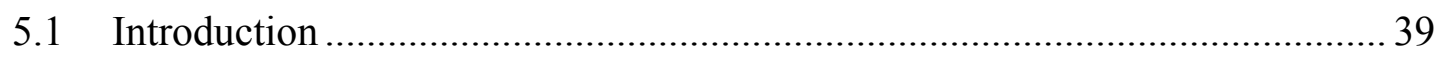

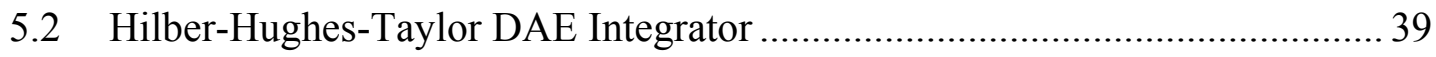

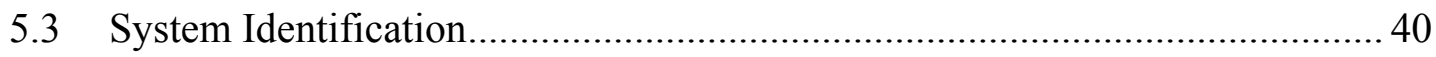

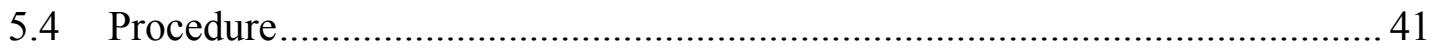

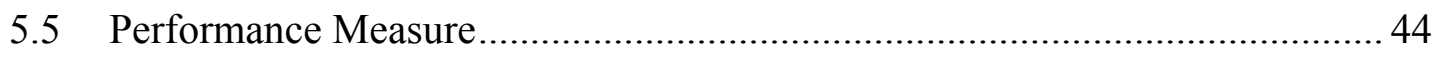

5.6 Linear System Identification Results .............................................................. 44

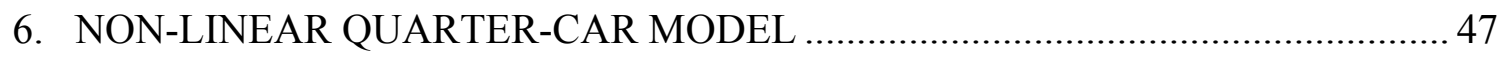

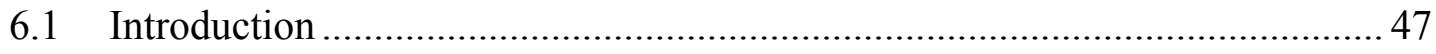

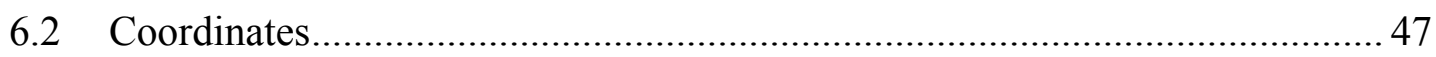

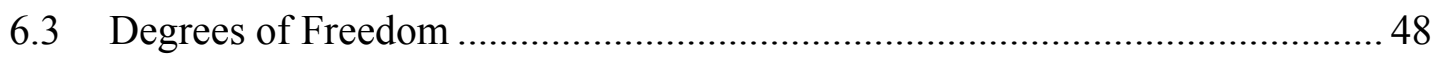

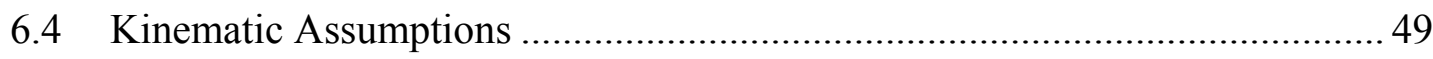

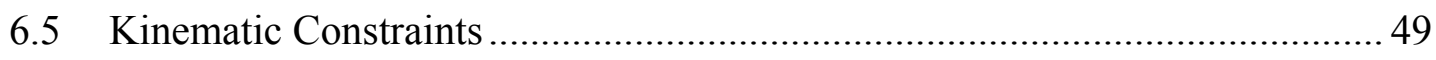

6.5.1 Translational Joint ....................................................................... 50

6.5.2 Revolute Joint A ................................................................................. 51

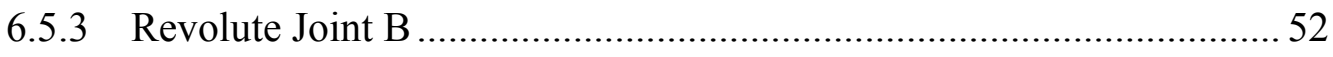


6.5.4 Revolute-Translational Joint PQ .............................................. 54

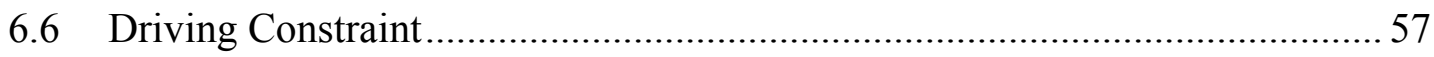

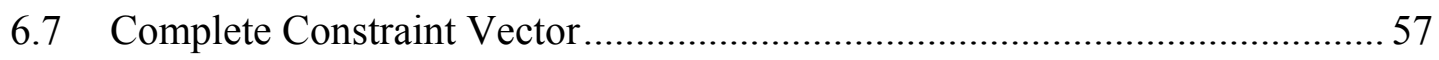

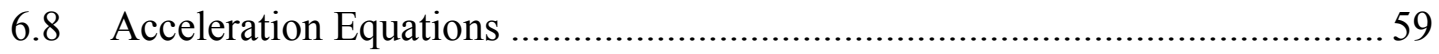

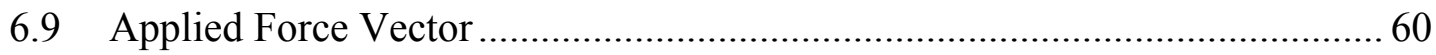

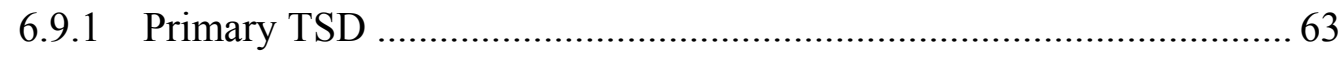

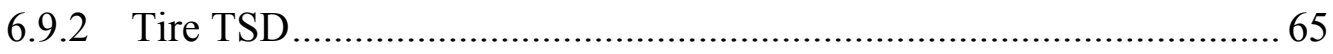

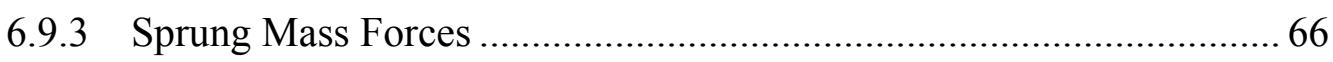

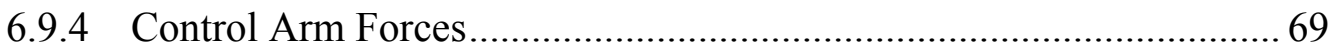

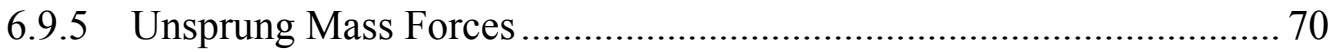

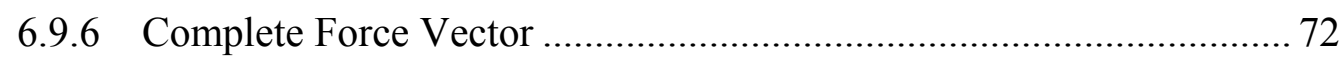

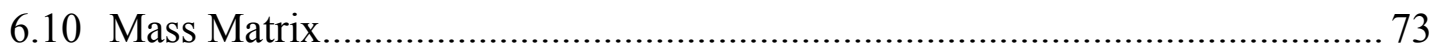

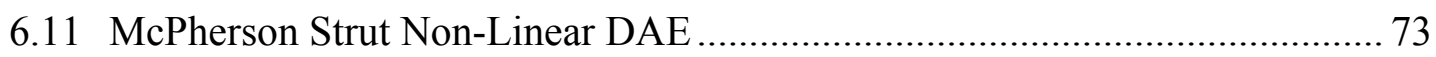

7. NON-LINEAR QUARTER-CAR MODEL SYSTEM IDENTIFICATION ............ 75

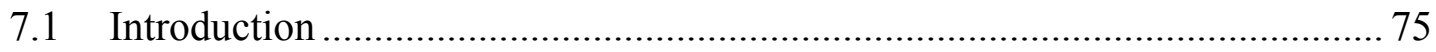

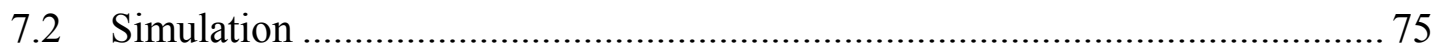

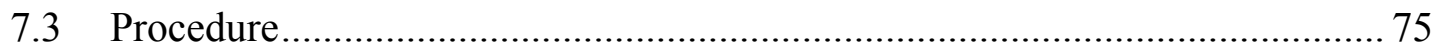

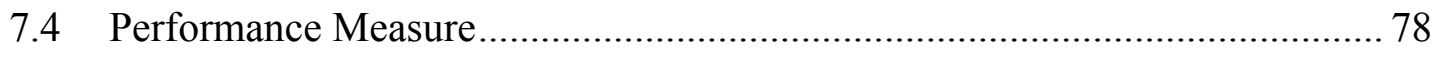

7.5 Non-Linear System Identification Results ............................................. 78

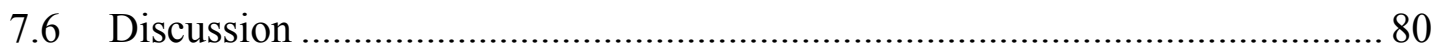

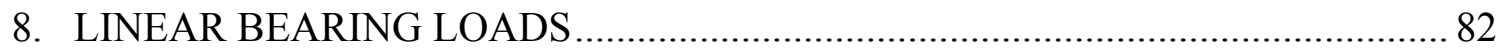

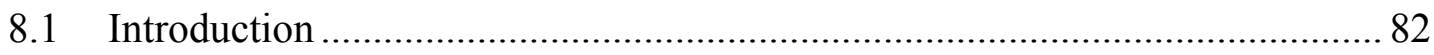

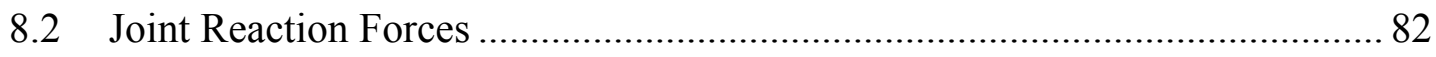

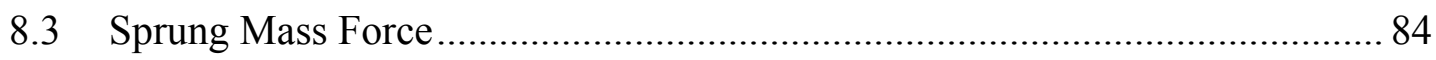

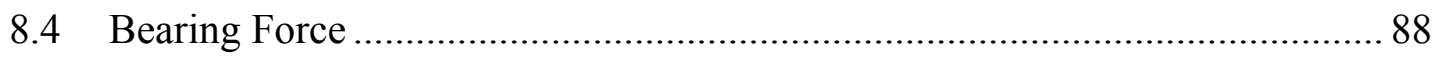

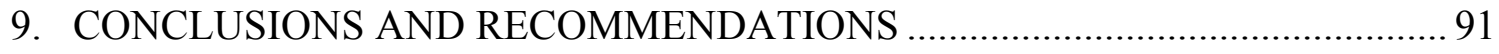

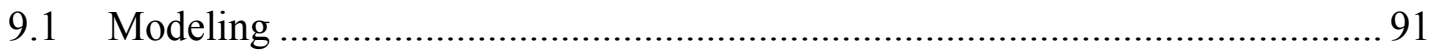




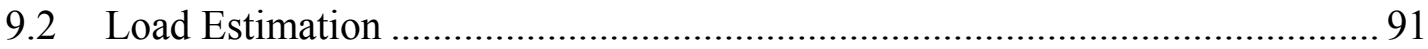

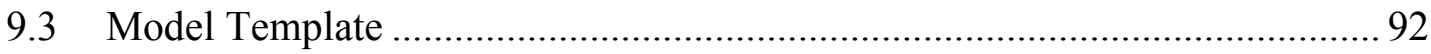

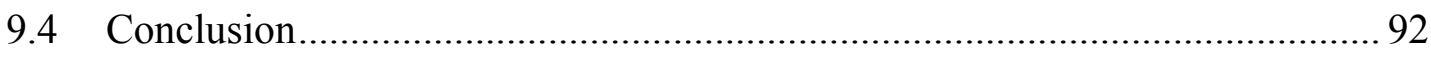

9.5 Recommendations for Future Research ……………................................... 92

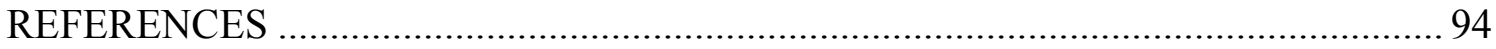

Appendix A - Control Arm and Unsprung Mass Moments of Inertia ............................ 96

Appendix B - Bearing Loads with Simplified Constraints ........................................... 102 


\section{LIST OF FIGURES}

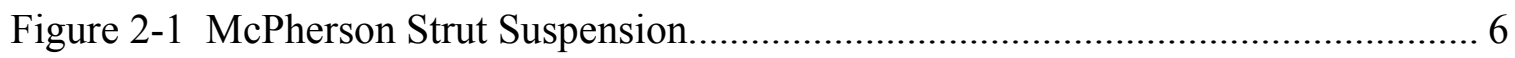

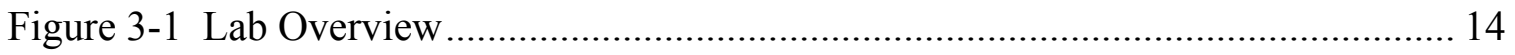

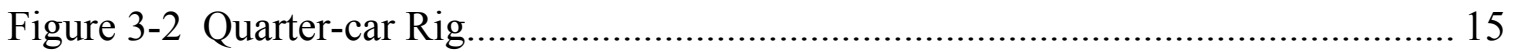

Figure 3-3 Hydraulic Equipment ............................................................................ 16

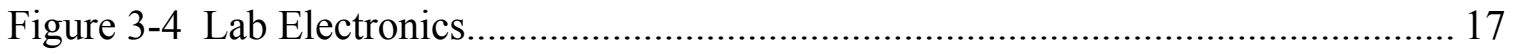

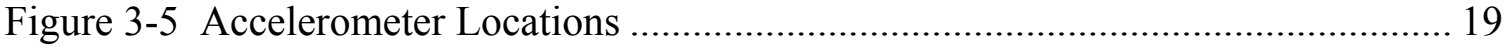

Figure 3-6 Accelerometer and Mounting Stud ............................................................. 20

Figure 3-7 LVDT Measured Pan Position vs. Time (0-8 Sec) ...................................... 22

Figure 3-8 LVDT Measured Pan Position vs. Time (5-6 Sec) .................................... 23

Figure 3-9 Measured Sprung and Unsprung Mass Acceleration vs. Time (0-8 Sec) ...... 23

Figure 3-10 Measured Sprung and Unsprung Mass Acceleration vs. Time (5-6 Sec) ... 24

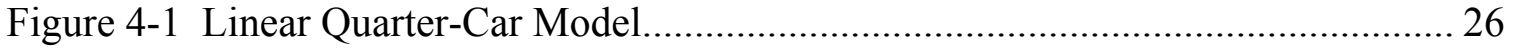

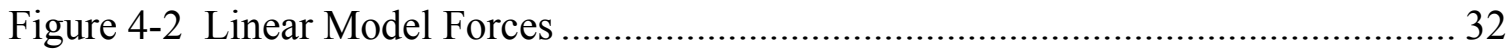

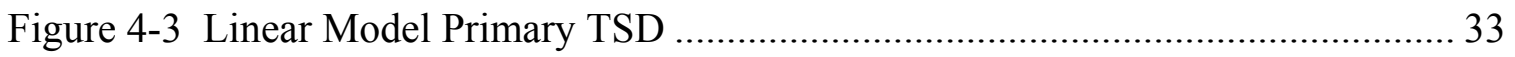

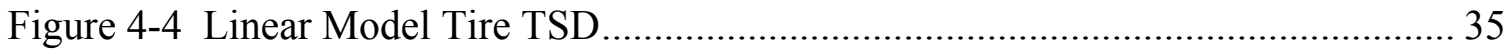

Figure 4-5 Linear Model Sprung Mass Forces ........................................................... 36

Figure 4-6 Linear Model Unsprung Mass Forces ................................................... 36

Figure 5-1 Linear Model Initial Parameter Estimates................................................ 43

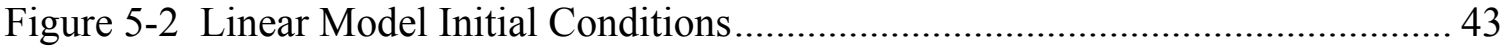

Figure 5-3 Experimental vs. Simulated Linear Model Acceleration with Non-Optimized

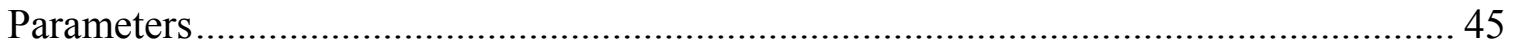

Figure 5-4 Experimental vs. Simulated Linear Model Acceleration with Optimized

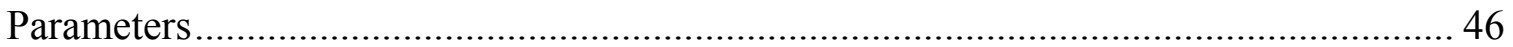

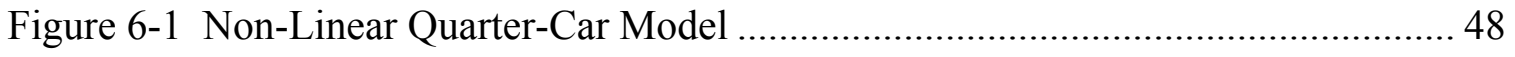

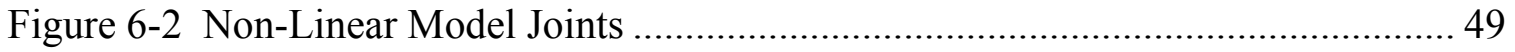

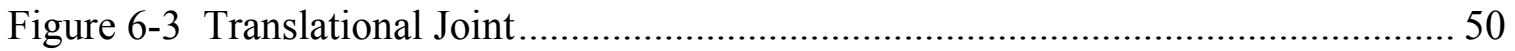

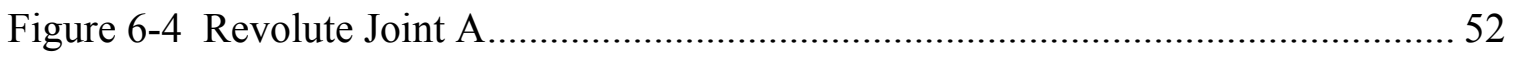

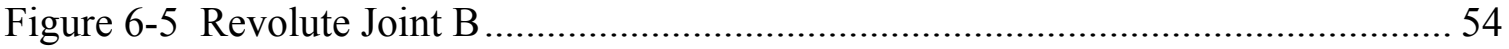

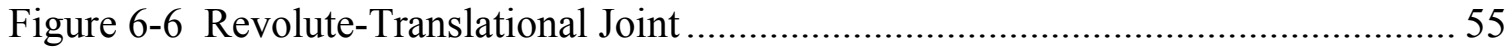

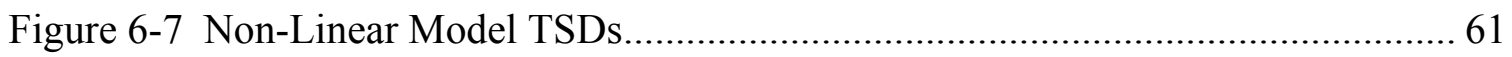




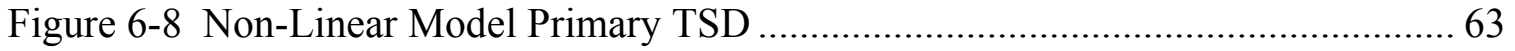

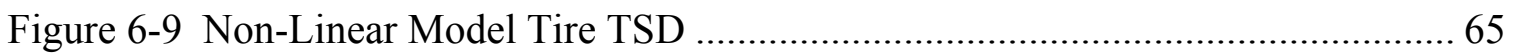

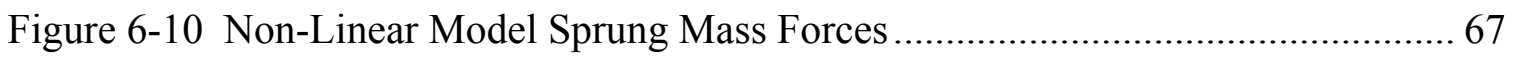

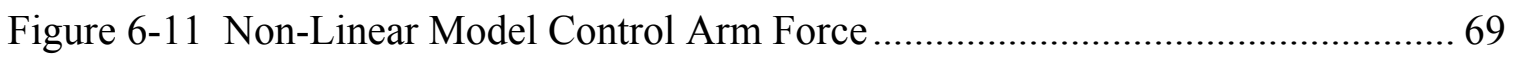

Figure 6-12 Non-Linear Model Unsprung Mass Forces............................................. 70

Figure 7-1 Non-Linear Model Initial Parameter Estimates ....................................... 76

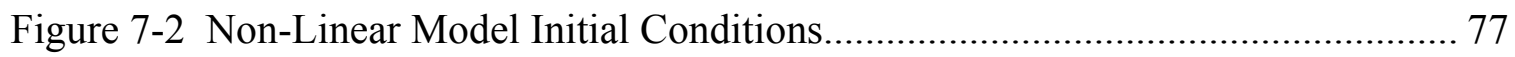

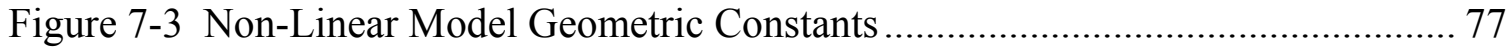

Figure 7-4 Experimental vs. Simulated Non-Linear Model Acceleration with Non-

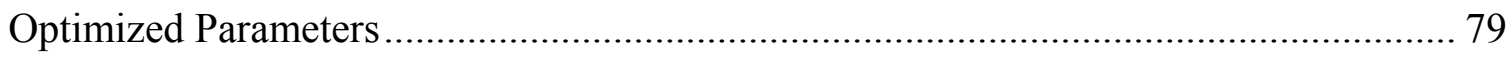

Figure 7-5 Experimental vs. Simulated Non-Linear Model Acceleration with Optimized

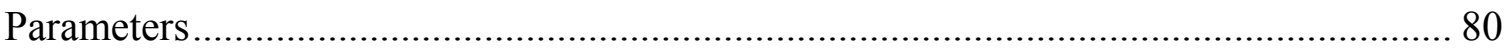

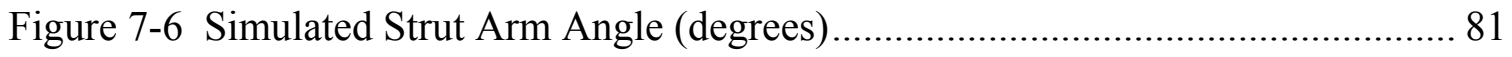

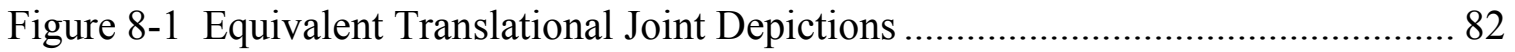

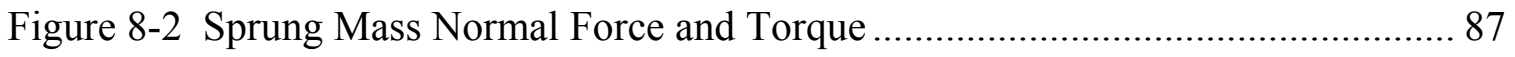

Figure 8-3 Simplified Quarter-car Bearing Reactions ............................................. 89

Figure 8-4 Upper and Lower Quarter-car Bearing Forces......................................... 89 


\section{LIST OF TABLES}

Table 2-1 Literature Search Matrix........................................................................... 5

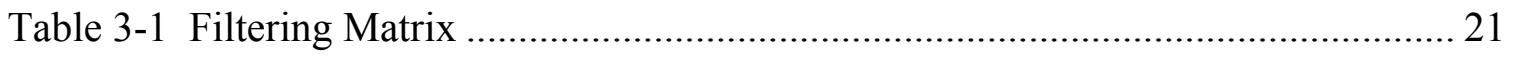

Table 5-1 Linear Model Optimal Parameters ........................................................... 45

Table 7-1 Non-Linear Model Optimal Parameters ........................................................ 80

Table 8-1 Non-Linear Model Optimized Parameters For Load Prediction ..................... 86 


\section{LIST OF SYMBOLS}

Symbol

$\boldsymbol{\Phi}^{\mathrm{t}} \quad$ Translational joint constraint vector

$\boldsymbol{\Phi}^{\mathrm{RA}} \quad$ Revolute joint A constraint vector

$\boldsymbol{\Phi}^{\mathrm{RB}} \quad$ Revolute joint B constraint vector

$\Phi^{\mathrm{RT}} \quad$ Revolute translational joint constraint

$\boldsymbol{\Phi}^{\mathrm{K}} \quad$ Kinematic constraint vector

$\Phi^{\mathrm{D}} \quad$ Driving constraint

Ф Complete constraint vector

$\Phi_{\mathbf{q}} \quad$ Constraint Jacobian

$\boldsymbol{\Phi}_{\mathrm{tt}} \quad$ Vector containing the second derivative of the constraint vector with respect to time

$\boldsymbol{\Phi}_{\mathrm{qt}} \quad$ Matrix containing the derivative of each element of the constraint Jacobian matrix with respect to time

$\left(\boldsymbol{\Phi}_{\mathrm{q}} \dot{\mathbf{q}}\right)_{\mathrm{q}} \quad$ Matrix containing the Jacobian of the product of the constraint Jacobian and the generalized velocity vector

$\boldsymbol{\alpha} \quad$ Vector of acceleration equations

q Generalized position vector $(\mathrm{m})$

$\dot{\mathbf{q}} \quad$ Generalized velocity vector $(\mathrm{m} / \mathrm{s})$

$\ddot{\mathbf{q}} \quad$ Generalized acceleration vector $\left(\mathrm{m} / \mathrm{s}^{2}\right)$

$\mathbf{Q}^{\mathbf{A}} \quad$ Applied force vector $(\mathrm{N})$ 
$\mathrm{k}_{\mathrm{p}} \quad$ Primary suspension stiffness $(\mathrm{N} / \mathrm{m})$

$\mathrm{n}_{\mathrm{p}} \quad$ Primary suspension damping $(\mathrm{N} /(\mathrm{m} / \mathrm{s}))$

$\mathrm{k}_{\mathrm{t}} \quad$ Tire stiffness $(\mathrm{N} / \mathrm{m})$

$\mathrm{n}_{\mathrm{t}} \quad$ Tire damping $(\mathrm{N} /(\mathrm{m} / \mathrm{s}))$

$\mathrm{F}^{\mathrm{P}} \quad$ Primary TSD force $(\mathrm{N})$

$\mathrm{F}^{\mathrm{T}} \quad$ Tire TSD force $(\mathrm{N})$

$\lambda \quad$ Vector of Lagrange multipliers

$s \phi_{\mathrm{i}}, c \phi_{\mathrm{i}} \quad$ Short-hand notation for sine and cosine of $\phi_{\mathrm{i}}$

X, Y Global reference frame

$\mathbf{r}_{\mathrm{S}} \quad \mathrm{CG}(\mathrm{m})$

$x_{\mathrm{S}}, y_{\mathrm{S}} \quad$ Global representation of the sprung mass position - components of $\mathbf{r}_{\mathrm{S}}(\mathrm{m})$

Global representation of the vector from the global origin to the control arm

$\mathbf{r}_{\mathrm{C}} \quad \mathrm{CG}(\mathrm{m})$

$x_{\mathrm{C}}, y_{\mathrm{C}} \quad$ Global representation of the control arm position - components of $\mathbf{r}_{\mathrm{C}}(\mathrm{m})$

Global representation of the vector from the global origin to the unsprung mass CG $(\mathrm{m})$

$x_{\mathrm{U}}, y_{\mathrm{U}} \quad(\mathrm{m})$

$y_{\mathrm{A}} \quad$ Global representation of the wheel pan position $(\mathrm{m})$

$x_{\mathrm{S}}{ }^{\prime}, y_{\mathrm{S}}{ }^{\prime} \quad$ Local reference frame embedded at the sprung mass CG

$x_{\mathrm{C}}{ }^{\prime}, y_{\mathrm{C}}{ }^{\prime} \quad$ Local reference frame embedded at the control arm CG 
$x_{\mathrm{U}}{ }^{\prime}, y_{\mathrm{U}}{ }^{\prime} \quad$ Local reference frame embedded at the unsprung mass CG

$\mathbf{s}_{\mathrm{S}}^{\text {A }} \quad$ Local representation of the body fixed vector from the sprung mass CG to revolute joint $\mathrm{A}(\mathrm{m})$

$\mathrm{s}_{\mathrm{Sx}}^{\prime \mathrm{A}}, s_{\mathrm{Sy}}^{\prime \mathrm{A}} \quad$ Local $x_{\mathrm{S}}^{\prime}$ and $y_{\mathrm{S}}^{\prime}$ components of the body fixed vector $\mathbf{s}_{\mathrm{S}}^{\prime \mathrm{A}}(\mathrm{m})$

$\mathbf{s}_{\mathrm{C}}^{\mathrm{A}} \quad$ Local representation of the body fixed vector from the control arm CG to revolute joint A $(\mathrm{m})$

$s_{\mathrm{Cx}}^{\prime \mathrm{A}},{s_{\mathrm{Cy}}^{\prime}}^{\mathrm{A}} \quad$ Local $x_{\mathrm{C}}{ }^{\prime}$ and $y_{\mathrm{C}}{ }^{\prime}$ components of the body fixed vector $\mathbf{s}_{\mathrm{C}}^{\prime \mathrm{A}}(\mathrm{m})$

$\mathbf{s}_{\mathrm{C}}^{\mathrm{B}} \quad$ Local representation of the body fixed vector from the control arm CG to revolute joint B $(\mathrm{m})$

$s_{\mathrm{CX}}^{\prime \mathrm{B}},{s_{\mathrm{Cy}}^{\prime}}^{\mathrm{B}} \quad$ Local $x_{\mathrm{C}}{ }^{\prime}$ and $y_{\mathrm{C}}{ }^{\prime}$ components of the body fixed vector $\mathbf{s}_{\mathrm{C}}^{\prime \mathrm{B}}(\mathrm{m})$

$\mathbf{s}_{U}^{\prime B} \quad$ Local representation of the body fixed vector from the unsprung mass CG to revolute joint $B(\mathrm{~m})$

$s_{\mathrm{Ux}}^{\prime \mathrm{B}},{s_{\mathrm{Uy}}^{\prime}}^{\mathrm{B}} \quad$ Local $x_{\mathrm{U}}{ }^{\prime}$ and $y_{\mathrm{U}}{ }^{\prime}$ components of the body fixed vector $\mathbf{s}_{\mathrm{U}}^{\prime \mathrm{B}}(\mathrm{m})$

$\mathbf{s}_{\mathrm{S}}^{\mathrm{Q}} \quad$ Local representation of the body fixed vector from the sprung mass CG to revolute joint $\mathrm{Q}(\mathrm{m})$

$s_{\mathrm{Sx}}^{\prime \mathrm{Q}}, s_{\mathrm{Sy}}^{\prime \mathrm{Q}} \quad$ Local $x_{\mathrm{S}}^{\prime}$ and $y_{\mathrm{S}}^{\prime}$ components of the body fixed vector $\mathbf{s}_{\mathrm{S}}^{\prime \mathrm{Q}}(\mathrm{m})$

Local representation of the body fixed vector from the unsprung mass CG to $\mathbf{s}_{\mathrm{U}}^{\prime \mathrm{P}} \quad$ point $\mathrm{P}$ on strut's translation line $(\mathrm{m})$

$s_{\mathrm{Ux}}^{\prime \mathrm{P}}, s_{\mathrm{Uy}}^{\prime \mathrm{P}} \quad$ Local $x_{\mathrm{U}}^{\prime}$ and $y_{\mathrm{U}}^{\prime}$ components of the body fixed vector $\mathbf{s}_{\mathrm{U}}^{\prime \mathrm{P}} \rightarrow s_{\mathrm{Ux}}^{\prime \mathrm{P}}={s_{\mathrm{Ux}}^{\prime}}^{\prime \mathrm{B}}(\mathrm{m})$

$\phi_{\mathrm{S}}, \phi_{\mathrm{C}}, \phi_{\mathrm{U}} \quad$ Sprung mass, control arm, and unsprung mass global orientation angle (rad)

$\mathbf{A}_{\mathrm{S}} \quad$ Transformation matrix from the local $x_{\mathrm{S}}^{\prime}-y_{\mathrm{S}}^{\prime}$ sprung mass reference frame to the global reference frame

$\mathbf{A}_{\mathrm{C}} \quad$ Transformation matrix from the local $x_{\mathrm{C}}{ }^{\prime}-y_{\mathrm{C}}{ }^{\prime}$ control arm reference frame to the global reference frame

$\mathbf{A}_{U} \quad$ Transformation matrix from the local $x_{\mathrm{U}}{ }^{\prime}-y_{\mathrm{U}}{ }^{\prime}$ unsprung mass reference frame to the global reference frame

$\mathbf{w}_{\mathrm{U}} \quad$ Unit vector orthogonal to the strut's line of translation in the negative $x_{\mathrm{U}}^{\prime}$ direction 


\begin{tabular}{|c|c|}
\hline $\mathbf{d}_{\mathrm{PQ}}$ & $\begin{array}{l}\text { Vector along the strut's line of translation from point } \mathrm{P} \text { to revolute joint } \mathrm{Q} \\
(\mathrm{m})\end{array}$ \\
\hline $\mathrm{C}$ & Magnitude of the projection of $\mathbf{d}_{\mathrm{PQ}}$ in the $\mathbf{w}_{\mathrm{U}}$-direction $(\mathrm{m})$ \\
\hline$\Delta \mathrm{d}_{\mathrm{PQ}}$ & Primary TSD compression $(\mathrm{m})$ \\
\hline $\mathrm{d}_{\mathrm{PQ}_{\mathrm{I}}}$ & Initial primary TSD length - primary spring free length $(\mathrm{m})$ \\
\hline $\mathrm{d}_{\mathrm{PQ}_{\mathrm{D}}}$ & Displaced primary TSD length $(\mathrm{m})$ \\
\hline$\dot{\mathbf{d}}_{\mathrm{PQ}}$ & Primary TSD compression velocity vector $(\mathrm{m} / \mathrm{s})$ \\
\hline SIGN & $\begin{array}{l}\text { Positive one if primary TSD is compressing and negative one if primary TSD } \\
\text { is expanding }\end{array}$ \\
\hline$\Delta \mathrm{d}_{\mathrm{AU}}^{\mathrm{y}}$ & Tire TSD compression $(\mathrm{m})$ \\
\hline $\mathrm{d}_{\mathrm{AU}_{\mathrm{I}}}^{\mathrm{y}}$ & $\begin{array}{l}\text { Initial tire TSD length - distance from acutuator origin to unsprung mass CG } \\
\text { when tire is not deflected }(\mathrm{m})\end{array}$ \\
\hline$\Delta \dot{\mathrm{d}}_{\mathrm{AU}}^{\mathrm{y}}$ & Tire TSD compression velocity $(\mathrm{m} / \mathrm{s})$ \\
\hline$\phi_{\text {Strut }}$ & Angle between the global X-axis and the strut's line of translation (rad) \\
\hline$\psi_{\mathrm{S}}$ & Non-fixed angle between vector $\mathbf{s}_{\mathrm{S}}^{\prime \mathrm{Q}}$ and $\mathrm{r}_{\mathrm{s}}^{\mathrm{P}}(\mathrm{rad})$ \\
\hline$\beta_{\mathrm{S}}$ & Fixed angle between the global $\mathrm{X}$-axis and vector $\mathbf{s}_{\mathrm{S}}^{\mathrm{Q}}(\mathrm{rad})$ \\
\hline$r_{S}^{p}$ & $\begin{array}{l}\text { Length of the line perpendicular to } F^{P} \text { from the sprung mass } C G \text { to } F^{P} \rightarrow \\
\text { sprung mass moment arm }(m)\end{array}$ \\
\hline$r_{U}^{\mathrm{T}}$ & $\begin{array}{l}\text { Length of the line perpendicular to } \mathrm{F}^{\mathrm{T}} \text { from the unsprung mass } \mathrm{CG} \text { to } \mathrm{F}^{\mathrm{T}} \rightarrow \\
\text { tire moment } \operatorname{arm}(\mathrm{m})\end{array}$ \\
\hline$\sum \mathrm{F}_{\mathrm{Sx} \rightarrow}^{\mathrm{A}}$ & Sum of forces applied to the sprung mass $\mathrm{CG}$ in the global X-direction $(\mathrm{N})$ \\
\hline$\sum \mathrm{F}_{\mathrm{Sy} \uparrow}^{\mathrm{A}}$ & Sum of forces applied to the sprung mass CG in the global Y-direction $(\mathrm{N})$ \\
\hline$\sum$ & Sum of counter c \\
\hline
\end{tabular}


$\mathbf{F}_{\mathrm{S}}^{\mathrm{A}} \quad$ Sprung mass applied force vector $(\mathrm{N})$

$\sum \mathrm{F}_{\mathrm{Cx} \rightarrow}^{\mathrm{A}} \quad$ Sum of forces applied to the control arm CG in the global X-direction (N)

$\sum \mathrm{F}_{\mathrm{Cy} \uparrow}^{\mathrm{A}} \quad$ Sum of forces applied to the control arm CG in the global $\mathrm{Y}$-direction $(\mathrm{N})$

$\sum \tau_{\mathrm{Cu}}^{\mathrm{A}} \quad$ Sum of counter clockwise torque applied about the control arm CG $(\mathrm{N} / \mathrm{m})$

$\mathbf{F}_{\mathrm{C}}^{\mathrm{A}} \quad$ Control arm applied force vector $(\mathrm{N})$ $\sum \mathrm{F}_{\mathrm{Ux} \rightarrow}^{\mathrm{A}} \quad \begin{aligned} & \text { Sum of forces applied to the unsprung mass } \mathrm{CG} \text { in the global } \mathrm{X} \text {-direction } \\ & (\mathrm{N})\end{aligned}$

$\sum \mathrm{F}^{\mathrm{A}} \quad$ Sum of forces applied to the unsprung mass $\mathrm{CG}$ in the global $\mathrm{Y}$-direction $(\mathrm{N})$

$\sum \tau^{\mathrm{A}} \quad$ Sum of counter clockwise torque applied about the unsprung mass CG

$\sum \tau_{\mathrm{Uu}}^{\mathrm{A}} \quad(\mathrm{N} / \mathrm{m})$

$\mathbf{F}_{\mathrm{U}}^{\mathrm{A}} \quad$ Unsprung mass applied force vector $(\mathrm{N})$ $\sum \mathrm{F}_{\mathrm{Ay} \uparrow}^{\mathrm{A} \uparrow} \quad \begin{aligned} & \text { Sum of forces applied to the top edge of the actuator in the global Y-direction } \\ & (\mathrm{N})\end{aligned}$

M Mass matrix ( $\mathrm{kg})$

$\mathrm{m}_{\mathrm{S}} \quad$ Sprung mass $(\mathrm{kg})$

$\mathrm{m}_{\mathrm{C}} \quad$ Control arm mass $(\mathrm{kg})$

$\mathrm{m}_{\mathrm{U}} \quad$ Unsprung mass $(\mathrm{kg})$

$\mathrm{m}_{\mathrm{A}} \quad$ Actuator mass $(\mathrm{kg})$

$j_{\mathrm{S}} \quad$ Sprung mass moment of inertia $\left(\mathrm{kg} \cdot \mathrm{m}^{2}\right)$

$j_{\mathrm{C}} \quad$ Control arm moment of inertia $\left(\mathrm{kg} \cdot \mathrm{m}^{2}\right)$

$j_{\mathrm{U}} \quad$ Unsprung mass moment of inertia $\left(\mathrm{kg} \cdot \mathrm{m}^{2}\right)$ 
g Acceleration due to gravity $\left(\mathrm{m} / \mathrm{s}^{2}\right)$

$\mathrm{R}_{\mathrm{S}} \quad$ Sprung mass performance ratio $(\mathrm{dB})$

$\mathrm{R}_{\mathrm{U}} \quad$ Unsprung mass performance ratio (dB)

$\mathbf{F}_{i}^{\prime \prime k} \quad$ Joint reaction force $(\mathrm{N})$

$\mathbf{C}_{i} \quad$ Rotation matrix that transforms vectors in the joint reference frame ' $\mathrm{i}$ ' to the body reference frame

A Rotation matrix that transforms vectors in the body reference frame ' $\mathrm{i}$ ' to the global reference frame

$\boldsymbol{\Phi}_{\mathrm{r}_{\mathrm{i}}}^{k} \quad$ Partial Jacobian associated with constraint force and translational coordinates

$\lambda^{k} \quad$ Vector of Lagrange multipliers associated with joint $\mathrm{k}$

$\tau_{i}^{\prime \prime k} \quad$ Joint reaction torque $(\mathrm{N} / \mathrm{m})$

$\mathbf{s}_{i}^{\mathbf{P}^{P}} \quad$ Vector from body i's local reference frame to the joint reference frame $(\mathrm{m})$

$\mathbf{B}_{i} \quad$ Derivative of the rotation matrix with respect to its angle

$\boldsymbol{\Phi}_{\phi_{1}}^{k} \quad$ Partial Jacobian associated with constraint torque and rotational coordinate

$\mathbf{F}_{\mathrm{S}}^{\prime \prime} \quad$ Sprung mass translational joint reaction force $(\mathrm{N})$

$\mathbf{C}_{\mathrm{S}} \quad$ Sprung mass rotation matrix that transforms vector $\mathbf{s}_{\mathrm{S}}^{\prime P}$ in the translational joint reference frame to the sprung mass reference frame $\begin{array}{ll}\boldsymbol{\Phi}_{\mathrm{r}_{\mathrm{s}}} & \text { Jacobian of sprung mass constraint vector with respect to translational } \\ \text { coordinates }\end{array}$

$\tau_{\mathrm{S}}^{\prime \prime} \quad$ Sprung mass joint reaction torque $(\mathrm{N} / \mathrm{m})$

$\mathbf{s}_{\mathrm{S}}^{{ }^{P}} \quad$ Vector from the sprung mass $\mathrm{CG}$ to the translational joint reference frame (m)

$\mathbf{B}_{\mathrm{S}} \quad$ Derivative of $\mathbf{A}_{\mathrm{S}}$ with respect to $\phi_{\mathrm{S}}$ 


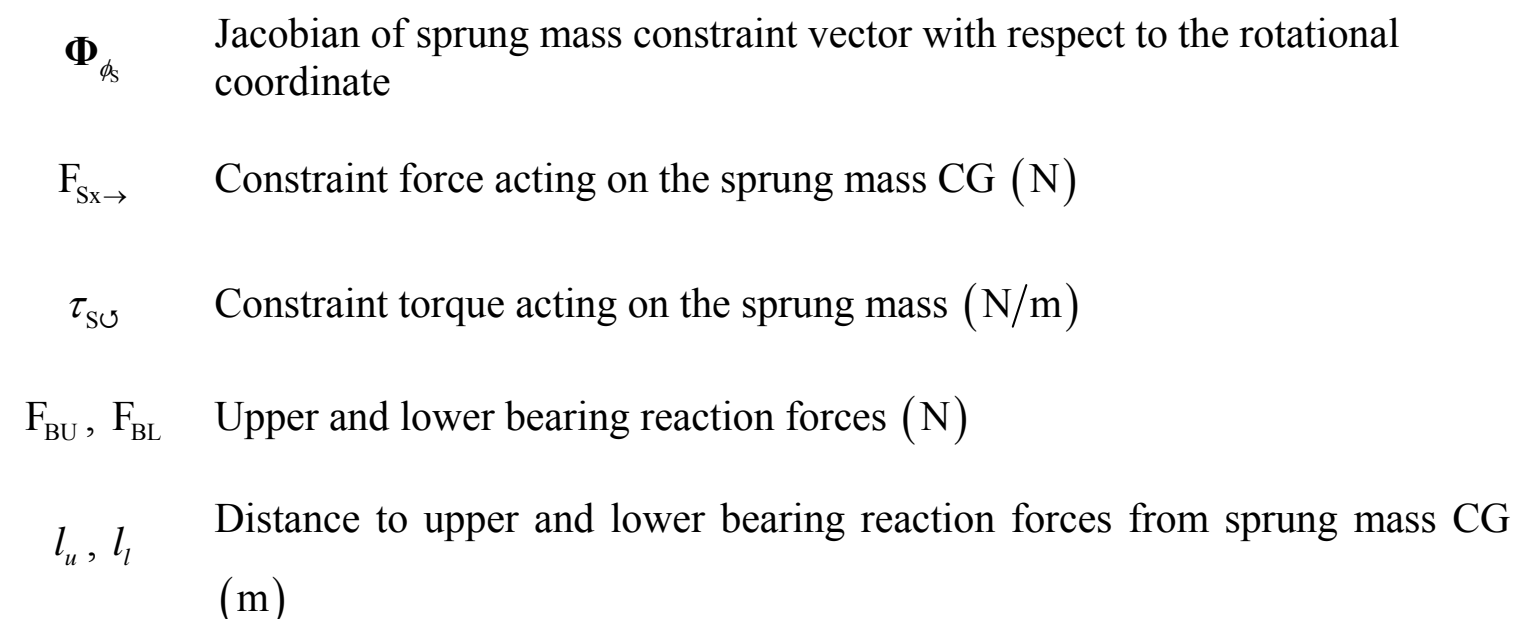




\section{INTRODUCTION}

This thesis addresses modeling, system identification, and various design aspects related to a McPherson strut suspension. As such, it has both theoretical and experimental components. A state-of-the-art quarter-car test rig with the front left McPherson strut suspension from a 2004 Porsche 996 Grand American Cup GS Class race car provides the experimental counterpoint to the theoretical models developed herein.

\subsection{Motivation}

Active and semi-active suspensions have the potential to increase vehicular comfort, performance, and safety (Gillespie [1]). For example, an active suspension may increase comfort by reducing the acceleration experienced by the sprung mass and, hence, by the vehicle occupants. An active suspension may increase the vehicle performance and safety of its occupants by minimizing the tire-pavement normal force fluctuation. By stabilizing this force, the dips in lateral adhesion associated with lower-than-usual tirepavement normal force can be avoided, which facilitates predictable, safe, and high performance handling characteristics. The effectiveness of active and semi-active suspensions is, in some cases, partly attributed to the controller's ability to predict the suspension's dynamic response. Thus, an accurate suspension model is necessary for the controller to predict the suspension's response.

Computer based quarter-car test models are often employed in vehicle dynamics studies as simplified and well-understood systems for such uses as testing new control strategies, designing new suspension systems, and analyzing ride dynamics. When available, quarter-car rigs are used to obtain relevant experimental data. A quarter-car test rig is an experimental platform which attempts to replicate the dynamics of one corner of a car. Having an accurate computer model of the test rig used is very valuable since it gives the researcher more flexibility in experimenting with various scenarios in the virtual world, helps design experiments, and helps perform extensive analysis. In this study the focus was on modeling and performing system identification on a quarter-car rig that, unlike typical quarter-car rigs, contains a full suspension system. The suspension under study is 
a McPherson strut suspension, but the quarter-car test rig, as it was designed, is capable of accommodating a multitude of different suspensions. As such, care must be taken so that a suspension that is capable of producing dynamic loads higher than that which the quarter-car test rig's linear bearings are rated for is not used. This load, termed here as the normal load, is that which is applied to the sprung mass plate's linear bearings perpendicular to the side which the suspension attaches to. Multibody dynamics models are capable of estimating the dynamic normal loads applied to the quarter-car rig's sprung mass, which provides additional motivation for the present study.

It is foreseeable that different suspensions will be used on the quarter-car test rig. It is expected that a non-linear quarter-car dynamics model that accurately simulates the original system and accounts for its constraints will provide more accurate results and offer increased capabilities compared to traditional linear single-axis quarter-car models. The term non-linear refers to the fact that, in addition to simulating its linear motion, the non-linear model accounts for the suspension's angular displacements. In order to take advantage of its increased accuracy and special capabilities, the quarter-car model must be clearly defined and easy to adapt to future changes in the system.

\subsection{Objectives}

The first objective is to formulate linear and non-linear multibody dynamic McPherson strut suspension models and compare their accuracy. For this study, the most accurate model is defined as the model that best predicts the experimental sprung mass and unsprung mass vertical accelerations.

The second objective is to use the non-linear multibody dynamics model to estimate the dynamic normal loads applied to the quarter-car rig's sprung mass plate and bearings.

The third objective is to clearly describe the formulation of the multibody dynamics nonlinear quarter-car McPherson strut suspension model so that it can be used as a template for future use. 


\subsection{Approach}

The non-linear motion of vehicular suspensions is often represented with a linear model. Among the reasons for such a modeling choice are ease and familiarity with linear modeling. Also, for non-linear suspensions that possess nearly linear motion, a linear model may represent the suspension's dynamics accurately enough for its intended application. However, the more non-linear a suspension's motion is, the less accurate a linear model is at predicting its dynamics. For moderately to highly non-linear suspensions a linear model may not be suitable for predicting dynamic response.

A McPherson strut suspension possesses non-linear motion, in part because its strut and control arm rotate. One way of quantifying the degree of non-linearity exhibited in a McPherson strut suspension is by examining its strut angle change throughout its range of motion. A race car-derived McPherson strut suspension, by design, does not facilitate much suspension travel and thus is minimally non-linear. Bearing this in mind, the approach used to determine the most accurate McPherson strut suspension model was to develop a linear model and a non-linear model using the same multibody dynamics method, and compare their respective performances. The performance comparison was carried out using optimal parameters found through system identification for each respective model.

Motion of the non-linear quarter-car model is completely described by a set of ordinary differential equations and algebraic equations associated with the constraints, leading to a set of differential algebraic equations (DAE) that must be solved to obtain system dynamics. In addition to obtaining the accelerations for the set of generalized coordinates used, solving the DAE will also yield Lagrange multipliers introduced to account for the algebraic constraints. These Lagrange multipliers are associated with the joint reaction forces. The Lagrange multipliers associated with the translational and rotational kinematic constraints of the sprung mass provide information about the normal reaction

force and the torque in the quarter-car rig's bearings. Thus the approach used to estimate the dynamic normal loads was to calculate them from the translational joint reaction force formula that utilizes Lagrange multipliers produced by the DAE simulation. 
The DAE describing the non-linear multibody dynamics of the quarter-car McPherson strut suspension is the mathematical basis needed to simulate and predict the dynamic response of the non-linear quarter-car test rig. A detailed description of the DAE system and the method used to solve it is provided in this study.

\subsection{Outline}

Chapter 2 presents results of the literature search. Chapter 3 explains the experimental quarter-car setup and the procedure used to collect data, and also contains experimental results. Chapter 4 develops the linear model approximation of the quarter-car McPherson strut suspension model. Chapter 5 presents background information on the integrator used to solve the DAE and on the system identification method used. The results of the system identification of the linear quarter-car McPherson strut suspension model developed in Chapter 4 are also presented here. Chapter 6 develops the non-linear multibody dynamics quarter-car McPherson strut suspension model. Chapter 7 discusses results from the system identification of the non-linear quarter-car McPherson strut suspension model developed in Chapter 6. Chapter 8 discusses the method used to determine the dynamic normal loads applied to the quarter-car rig's sprung mass. This chapter also presents bearing normal load results. Chapter 9 summarizes this work and provides recommendations for future research. 


\section{LITERATURE SEARCH}

This chapter presents a literature review related to McPherson strut suspension history and applications, multibody dynamics and quarter-car modeling, and simulation. Several sources were searched in conjunction with pertinent key words. Because both Macpherson and McPherson strut are popular spellings for this type of suspension, both were used in this search. Table 2-1 lists the number of articles found at each source containing these keywords:

Table 2-1 Literature Search Matrix

\begin{tabular}{|c|c|c|c|c|c|}
\hline & \multicolumn{5}{|c|}{ Key Words } \\
\hline Database (DB), Journal, or Thesis Source & 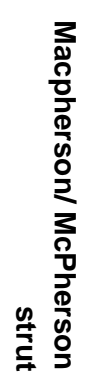 & 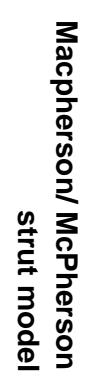 & 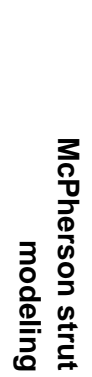 & 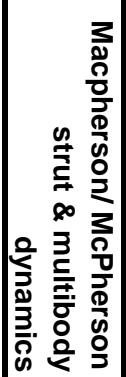 & 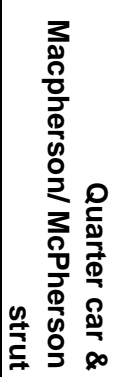 \\
\hline ANTE: Abstracts in New Technologies and Engineering (DB) & $3 / 4$ & $0 / 0$ & $0 / 0$ & $2 / 0$ & $2 / 0$ \\
\hline Engineering Village Compendex, Inspec \& NTIS (DBs) & $27 / 25$ & $2 / 0$ & $0 / 0$ & $1 / 1$ & $2 / 0$ \\
\hline CSA Technology (DB) & $19 / 14$ & $0 / 0$ & $0 / 0$ & $4 / 1$ & $2 / 0$ \\
\hline Mechanical \& Transportation Engineering Abstracts (DB) & $11 / 4$ & $0 / 0$ & $0 / 0$ & $2 / 0$ & $1 / 0$ \\
\hline SAE Technical Papers (DB) & $13 / 51$ & $0 / 0$ & $0 / 0$ & $1 / 4$ & $0 / 0$ \\
\hline Virginia Tech Addision Book Search & $0 / 0$ & $0 / 0$ & $0 / 0$ & $0 / 0$ & $0 / 0$ \\
\hline WorldCat dissertations and theses & $4 / 0$ & $0 / 0$ & $0 / 0$ & $0 / 0$ & $0 / 0$ \\
\hline
\end{tabular}

\subsection{McPherson Strut}

This section provides a historical and applications perspective on the McPherson strut suspension.

The McPherson strut suspension, designed in the late 1940s by Earl Steele Macpherson, was first used on the 1949 Ford Vedette (Gilles [2]). As such, it is a relatively new suspension configuration. Mantaras [3] states that the vast majority of current small- and medium-sized cars use this configuration. The McPherson strut suspension configuration 
consists of a lower control arm and telescopic strut attached to the body of the car and to the wheel carrier, which is also called an upright. It is often the case that the primary spring and damper are co-linear with the strut's line of translation.

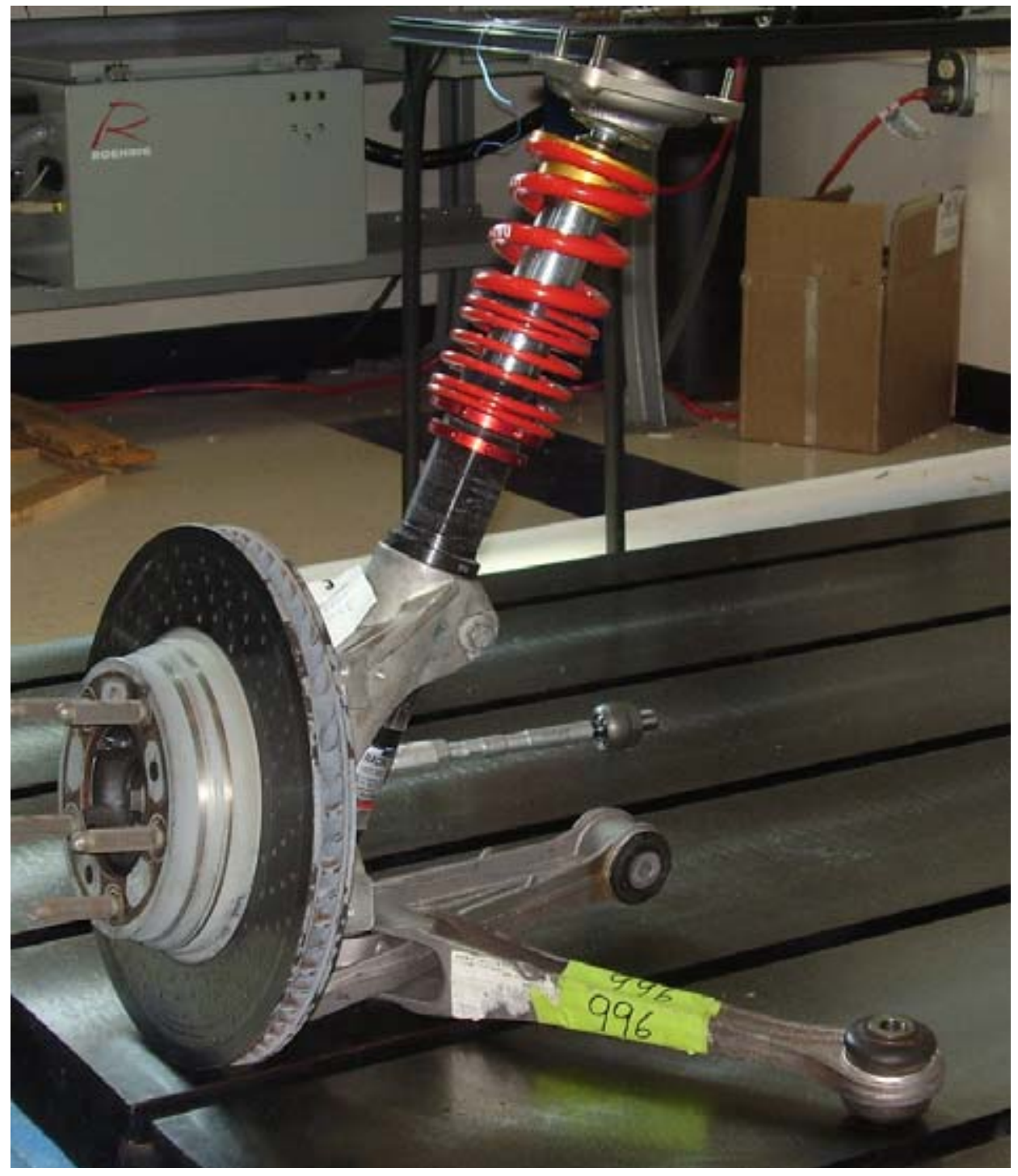

Figure 2-1 McPherson Strut Suspension

This configuration has several advantages. Gillespie [1] points out that its inherent Lshape aids with the packaging of transverse engines. Likewise, as Daniels [4] notes, its three mounting points can be widely spaced, thereby allowing this configuration to be made structurally efficient. Daniels also notes that camber angle change with suspension travel is small. A final advantage is the ease with which the strut can be replaced. 
Nevertheless, this type of suspension configuration also has some disadvantages. From a packaging standpoint, although beneficial for transverse engines, this configuration's high installation height limits the designer's ability to lower the hood height [1]. From a performance standpoint, Daniels notes that the effect of the rolling force increases the further the body rolls due to the roll center migration incurred with this suspension. Daniels also notes that roll center migration on a double wishbone suspension creates no serious problem [4]. Another performance compromise Milliken [5] mentions is that McPherson strut suspensions lose negative camber when the suspension travels upward.

Despite these disadvantages, manufacturers such as Porsche and BMW use this suspension to great effect in their road racing efforts. Likewise, consumer car makers such as Toyota and General Motors use them effectively on their passenger vehicles.

\subsection{Quarter-car Modeling}

This section discusses multibody dynamics background, methods, and previous work in quarter-car model construction.

Selection of the coordinate system is the first step in multibody dynamics modeling. DeJalon [6] discusses the implementation, advantages, and disadvantages associated with relative, reference point, and natural $2 \mathrm{D}$ coordinates. Relative coordinates describe the relative motion between two adjoining elements. For example, a revolute joint would be described by the angle between the elements it connects and a translational joint by the distance between connected elements. Constraint equations are generated from the vector equations that close kinematic loops. This system uses a low number of coordinates and is especially apt at describing open-chain configurations. However, mathematical formulation can be problematic, and although few coordinates are used the equations of motion they create can be computationally expensive. Additionally, pre- and postprocessing is required to determine each joint's absolute motion. 
Reference point coordinates specify element position and orientation by embedding a local coordinate system in that element, specifying the X-Y position of the local coordinate origin, and orientation angle between a local axis and the global inertial axis. Often local axes are denoted with a prime, for example $x^{\prime}$ or $y^{\prime}$. Constraint equations are written based on the type of motion each joint allows between adjacent elements. For example, a revolute joint would constrain both $\mathrm{X}$ and $\mathrm{Y}$ translation. Because the position and orientation of each element is directly specified, little pre- and post-processing are necessary to determine each joint's absolute motion. The main disadvantage of this system is that the large number of coordinates it requires can lengthen simulation time.

Natural coordinates specify two points on a body to determine its position and orientation in space. Constraints are written by rigid body condition (that is, the distance between two points on a rigid body is constant) or by joint constraints. This system is advantageous because it eliminates angular coordinates, and little pre- or post-processing is needed. However, this system requires a larger number of coordinates than relative coordinates would.

For this thesis, reference point coordinates are used because of their advantages. The kinematic and dynamic approach used in this study, including kinematic joint constraints, formulation of the differential equations of motion, and constraint reaction forces, follows the methodology described by Haug [7].

A ride frequency of approximately $4 \mathrm{~Hz}$ was observed from the experimental McPherson strut suspension. Milliken puts this result in context by providing useful insight as to what ride frequencies to expect from road-circuit race cars that employ aerodynamic down force packages. He mentions that ride frequencies between 3.0 and $5.0 \mathrm{~Hz}$ have proven successful for race cars with significant aerodynamic down force and that the front axle's ride frequency is higher than the rear. Given that the experimental suspension is from the front of a Porsche 996 race car that employs an aerodynamic down force package, the observed ride frequency of approximately $4 \mathrm{~Hz}$ is within expectation. 
Milliken also provides insight on how to use the observed ride frequency to estimate the total static deflection of the primary suspension and tire. Total static deflection is defined here as the change in vertical position of the sprung mass CG relative to the inertial reference frame that occurs when the sprung mass is placed on and statically supported by the suspension. Milliken presents the relationship between the natural ride frequency and static wheel deflection as:

$$
\omega_{n}=\frac{188}{\sqrt{\mathrm{x}}}(\mathrm{cyc} / \mathrm{min} .)
$$

In this equation $\omega_{n}$ is the ride natural frequency in cycles per minute and $\mathrm{x}$ is the static wheel deflection in inches. Changing the ride natural frequency from units of cycles per minute to cycles per second this equation becomes:

$$
\omega_{n}=\frac{188}{\sqrt{\mathrm{x}}}\left(\frac{\mathrm{cyc}}{\mathrm{min} .}\right)\left(\frac{\mathrm{min} .}{60 \mathrm{sec} .}\right)=\frac{3.13}{\sqrt{\mathrm{x}}}\left(\frac{\mathrm{cyc}}{\mathrm{sec} .}\right)
$$

Rearranging this equation, the static deflection given a particular ride frequency is:

$$
\mathrm{x}=\left(\frac{3.13}{\omega_{n}}\right)^{2}(\text { in })
$$

Given the observed experimental ride frequency of approximately $4 \mathrm{~Hz}$, the total static deflection this equation predicts is:

$$
x=\left(\frac{3.13}{4}\right)^{2}=0.61(\text { in })
$$


In meters the total static deflection is:

$$
\mathrm{x}=0.61(\text { in }) \times \frac{0.0254(\mathrm{~m})}{1(\text { in })}=0.0155(\mathrm{~m})
$$

Since the strut's line of translation is essentially along the unsprung mass's locus and thus the installation ratio is essentially 1 to 1 , this static deflection provides an estimation of the sum of initial strut and tire compression used in system identification.

Prior work in quarter-car model construction and control has also been reviewed. Rahnejat [8] discusses the formulation and analysis of a simplified multibody dynamics 2D McPherson strut suspension model. Gillespie [1] develops a linear quarter-car model to describe vehicle response properties such as sprung mass isolation and transmissibility. Inman [9] develops a base excitation model that can be used to represent a quarter-car suspension. Five semi-active control policies are tested on a full-scale 2 degree-offreedom quarter-car system incorporating a magneto-rheological damper in Goncalve's [10] experimental study. Mantaras [3] presents a set of kinematic constraints used to model a 3D McPherson strut suspension.

\subsection{Simulation}

This section discusses literature related to the simulation of multibody systems.

Once a multibody dynamics system is modeled with a set of differential algebraic equations of motion (DAE), the system can be simulated. There are several methods for solving DAE such as the Runge-Kutta algorithms discussed by Owren [11] and Laurent [12]. The method used in this thesis is the Hilber-Hughes-Taylor (HHT) method discussed by Negrut [13] and Cardona [14, 15]. This method was chosen to solve and simulate the non-linear model because, according to Negrut, the combination of numerical damping, A-stability, and second order accuracy make the HHT method attractive for solving non-linear kinematically constrained multibody dynamics DAE. 
Burden [16] defines a numerical method as A-stable if "its region R of absolute stability contains the entire left half-plane." For consistency, this method was also used to simulate the linear model.

There are several examples of prior work on correlating experimental and dynamically simulated suspensions. Trom [17] developed a multibody dynamics model of a midsized passenger car with front McPherson strut suspension in Dynamic Analysis and Design System (DADS) software. Dynamic simulations with this model are compared to corresponding experimental data. Park [18] used model data from Salaani [19] to create a full vehicle ADAMS simulation. This paper addressed how to refine kinematic steering and suspension models using measurement data. Ozdalyan [20] developed an ADAMS suspension model to replicate a Peugeot $605 \mathrm{McPherson}$ strut suspension that provides insight into how suspension parameters change in relation to each other. The wheel rate, camber angle, caster angle, steer angle, and track change with suspension travel is predicted in ADAMS and compared to the same experimentally-measured values obtained from the Peugeot. For this particular experimental McPherson strut suspension, the wheel rate, camber angle, caster angle, steer angle, and track changed approximately $3200 \mathrm{~N} / \mathrm{mm}, 1.05$ degrees, 0.4 degrees, 0.5 degrees, and $16 \mathrm{~mm}$, respectively, over 120 $\mathrm{mm}$ of suspension travel. This work provides evidence that a McPherson strut's change in camber is much larger than its change in caster. This evidence supports the notion that the majority of the kinematic non-linearity in a McPherson strut suspension can be captured using a front view 2D multibody dynamics model. 


\section{LABORATORY SETUP}

This chapter explains the experimental quarter-car test rig setup and the procedure used to collect data, and presents experimental results.

\subsection{Lab Overview}

Figure 3-1 shows an overview of the physical quarter-car rig, hydraulic actuation equipment, and electronic equipment used in the lab. Over the course of 2006, a state-ofthe-art quarter-car test rig was designed, constructed, and installed at the Institute for Advanced Learning and Research (IALR) for the Performance Engineering Research Lab (PERL). The rig has the potential to be used as a test bed for vehicular suspensions ranging from lightweight race cars to heavy off-road High-Mobility Multipurpose Wheeled Vehicles (HMMWV). This thesis used the front left McPherson strut suspension from a 2004 Porsche 996 Grand American Cup GS Class race car attached to the rig for experimental results. Sections below describe the experimental setup.

\subsection{Quarter-car Rig}

Figure 3-2 shows details of the quarter-car rig including the stationary frame, sprung mass, and unsprung mass. The stationary frame includes the quarter-car rig frame (most of which is painted in maroon) and the linear guides. The linear guides are equipped with NSK LH35 series linear bearings capable of supporting a 61,385 N dynamic normal load and 1,519 N-m static moment.

The sprung mass is defined here as the mass that is supported by the primary-ride spring. The sprung mass plate, adapter plate, and strut tower constitute the bulk of the sprung weight. Ballast weights were added to the sprung mass plate to approximate the left front corner weight of a Porsche 996 race car.

The unsprung mass is defined here as the mass that is not supported by the primary-ride spring. The strut and wheel assemblies constitute the bulk of the unsprung weight. The 
strut assembly includes the main and tender springs, damper, and upright. The wheel assembly is attached to the upright and consists of the brake disk, wheel, and tire. Suspension geometry was adjusted such that it matched the actual 996 geometry. Camber, caster, toe, and ride height were all brought within ranges found on the actual 996 race car.

Although at the time of this writing direct measurement of the rig's total corner weight was not possible, it has been estimated at $286 \mathrm{~kg}$. This estimation is based on direct measurement of suspension parts and computer solid modeling estimation of the sprung mass components. 


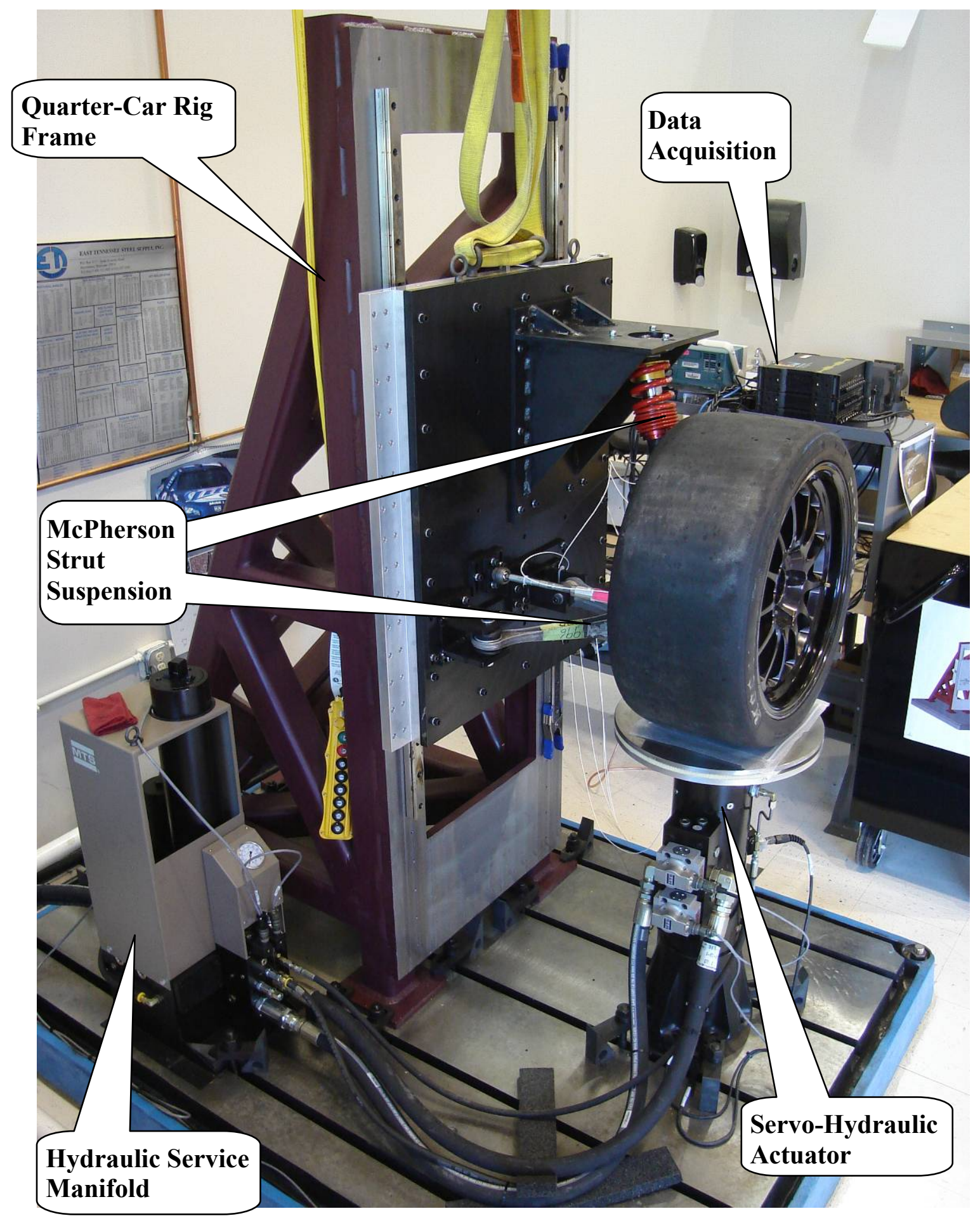

Figure 3-1 Lab Overview 


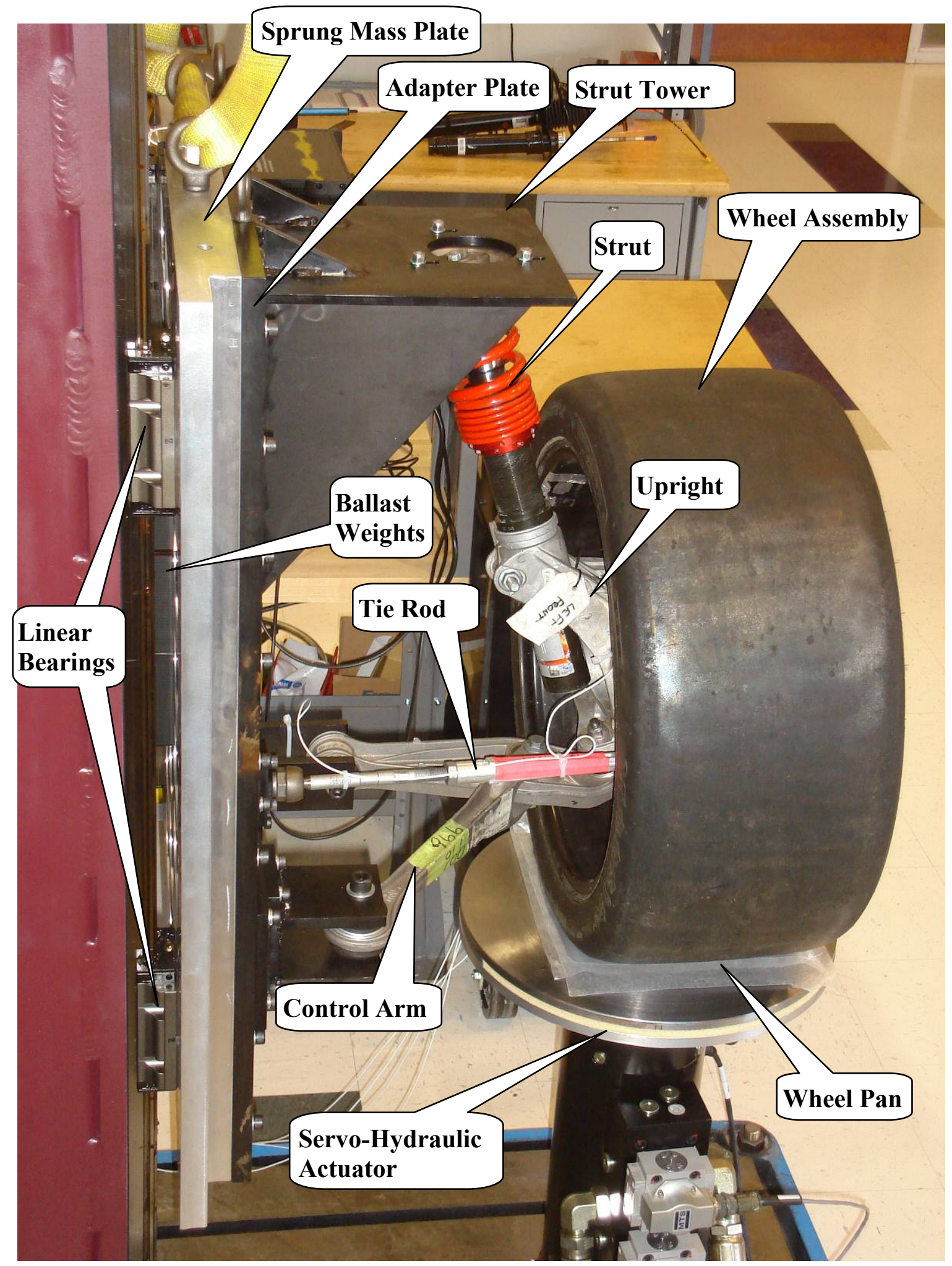

Figure 3-2 Quarter-car Rig 


\subsection{Hydraulic Driving Equipment}

Figure 3-3 shows an overview of the hydraulic driving equipment. The hydraulic driving equipment consists of an MTS Model 505.03 SilentFlo Hydraulic Power Supply, MTS 293.11 Hydraulic Service Manifold, Moog Model 252.25G-01 4-Port servo-valves, and MTS Model 248.03 Linear Hydraulic Actuator. The hydraulic power supply houses the main hydraulic fluid reservoir and

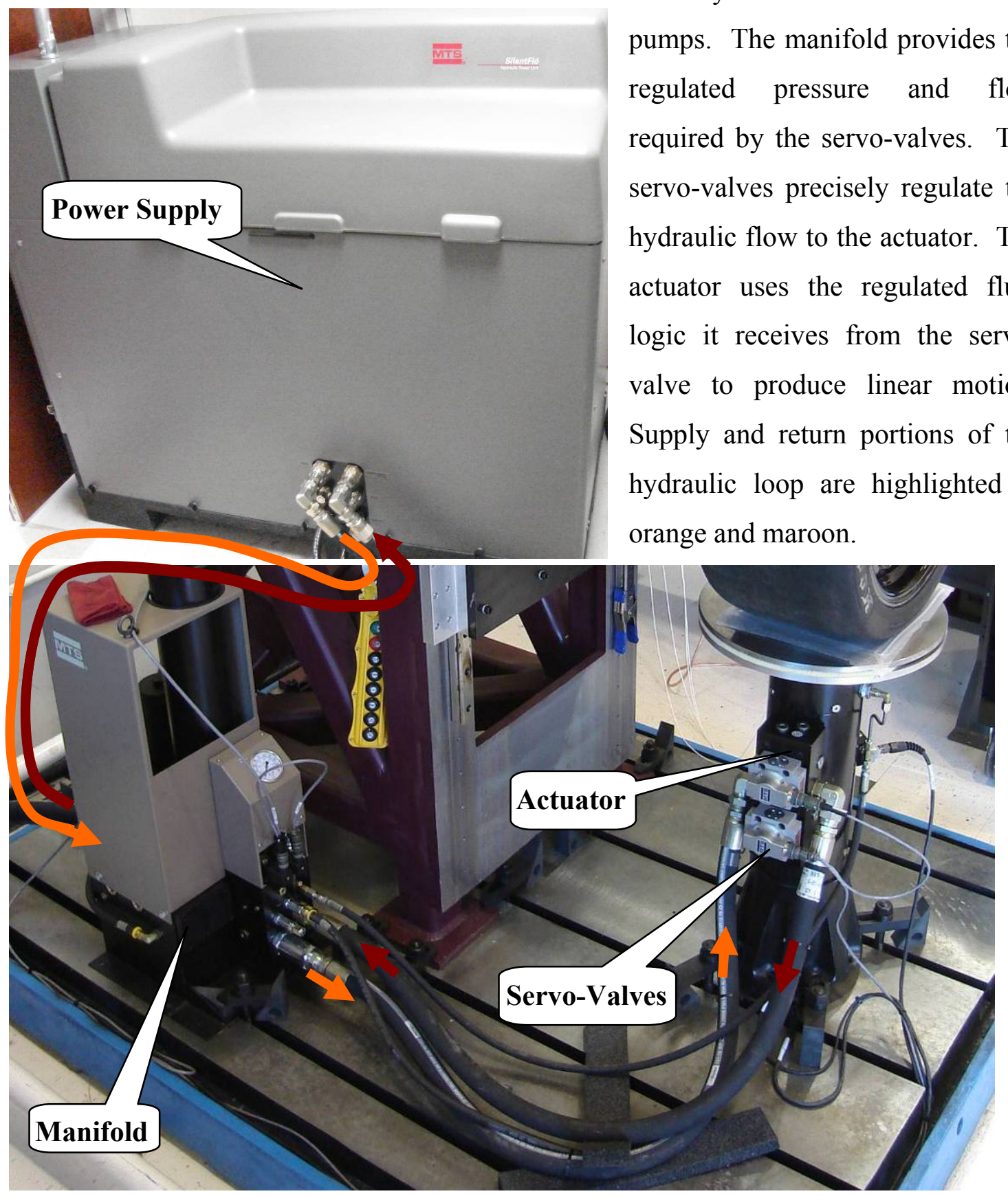

Figure 3-3 Hydraulic Equipment 


\subsection{Electronic Driving and Measuring Equipment}

Figure 3-4 shows an overview of the electronics used to drive and sense the quarter-car. To drive the quarter-car, a wheelpan displacement input file is created using Matlab. This displacement file is transferred from the lab computer to the dSpace AutoBox. The analog representation of the displacement signal is then transferred through the breakout box to the MTS Model 493.02 FlexTest SE Controller. This controller provides real-time closed-loop control while driving the servo-hydraulic actuator with the specified displacement.

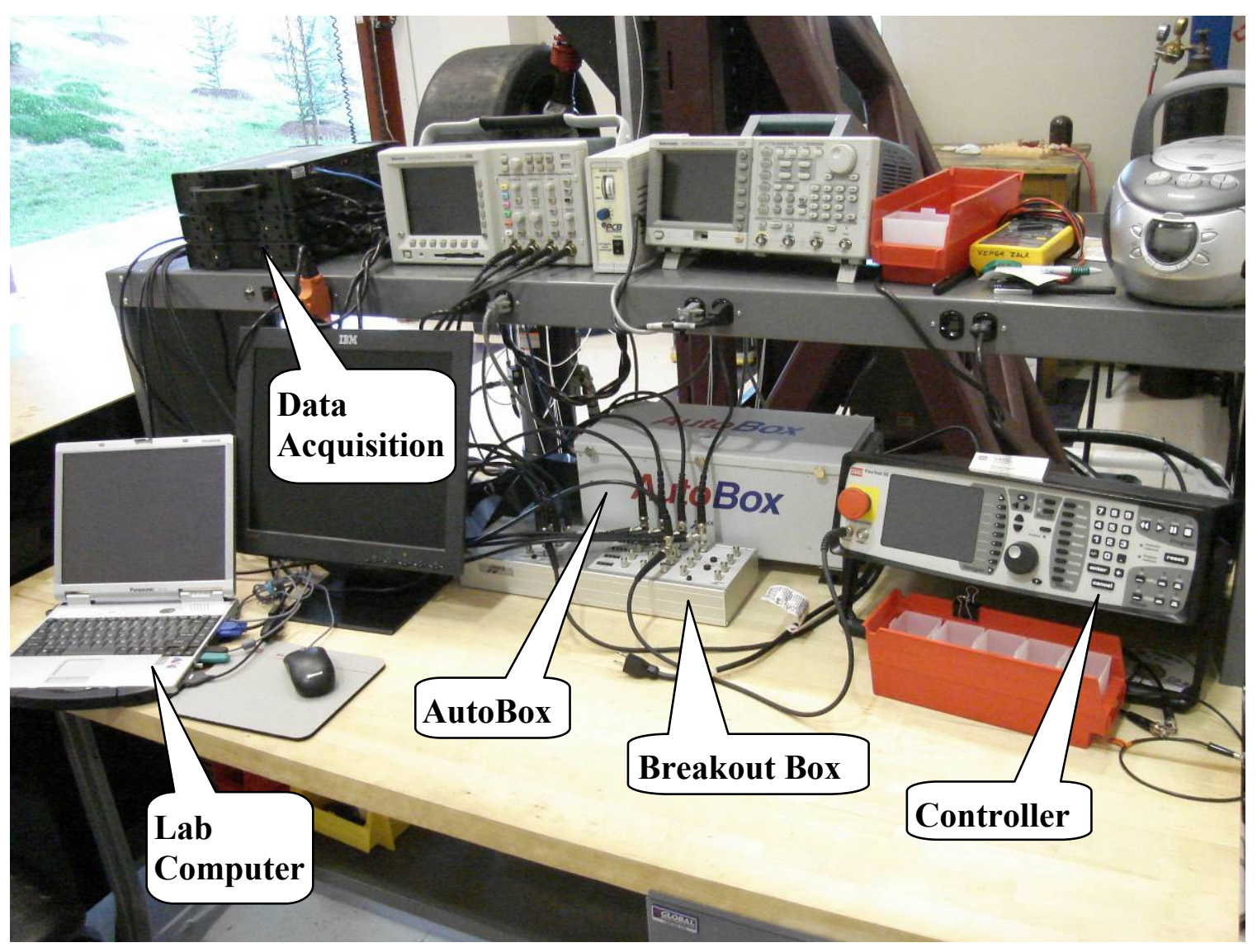

Figure 3-4 Lab Electronics

Together, IOtech's WaveBook/516E 16-bit 1 MHz Data Acquisition System and WBK18 8-channel signal conditioner provided the conduit between the measuring equipment and lab computer. Measured quantities include the wheel pan position and the sprung and unsprung mass accelerations. The pan position is measured with the inline linear- 
variable displacement transducer (LVDT) built into the hydraulic actuator. The accelerations were measured using PCB Piezotronic Model 333B40 10-g accelerometers. These piezoelectric accelerometers were calibrated to approximately $0.5 \mathrm{mV} / \mathrm{g}$. Figure $3-$ 5 shows where the accelerometers are located on the sprung mass and unsprung mass. The accelerometers are hard mounted to the sprung and unsprung masses using the small stud in Figure 3-6. 


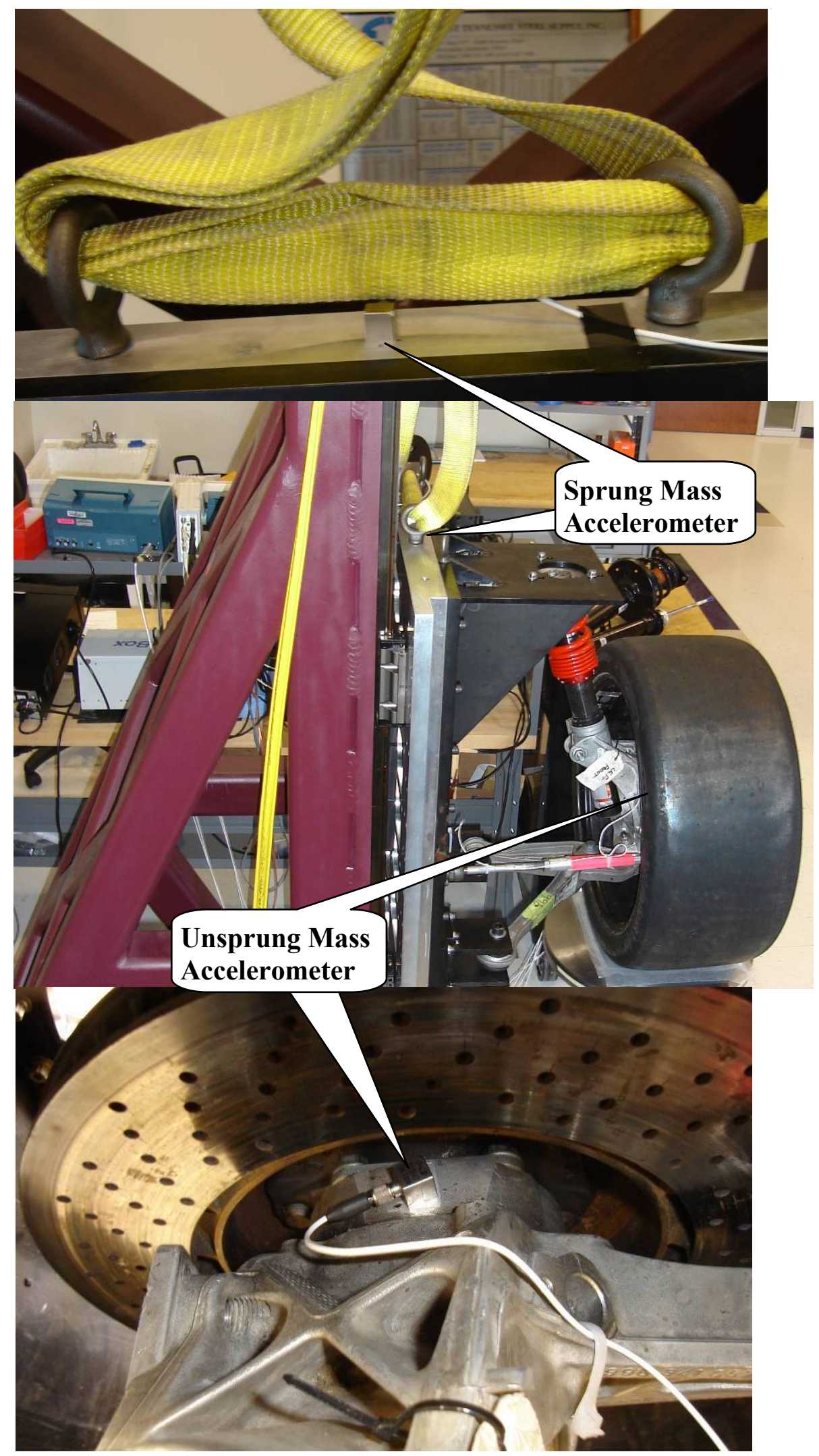

Figure 3-5 Accelerometer Locations 


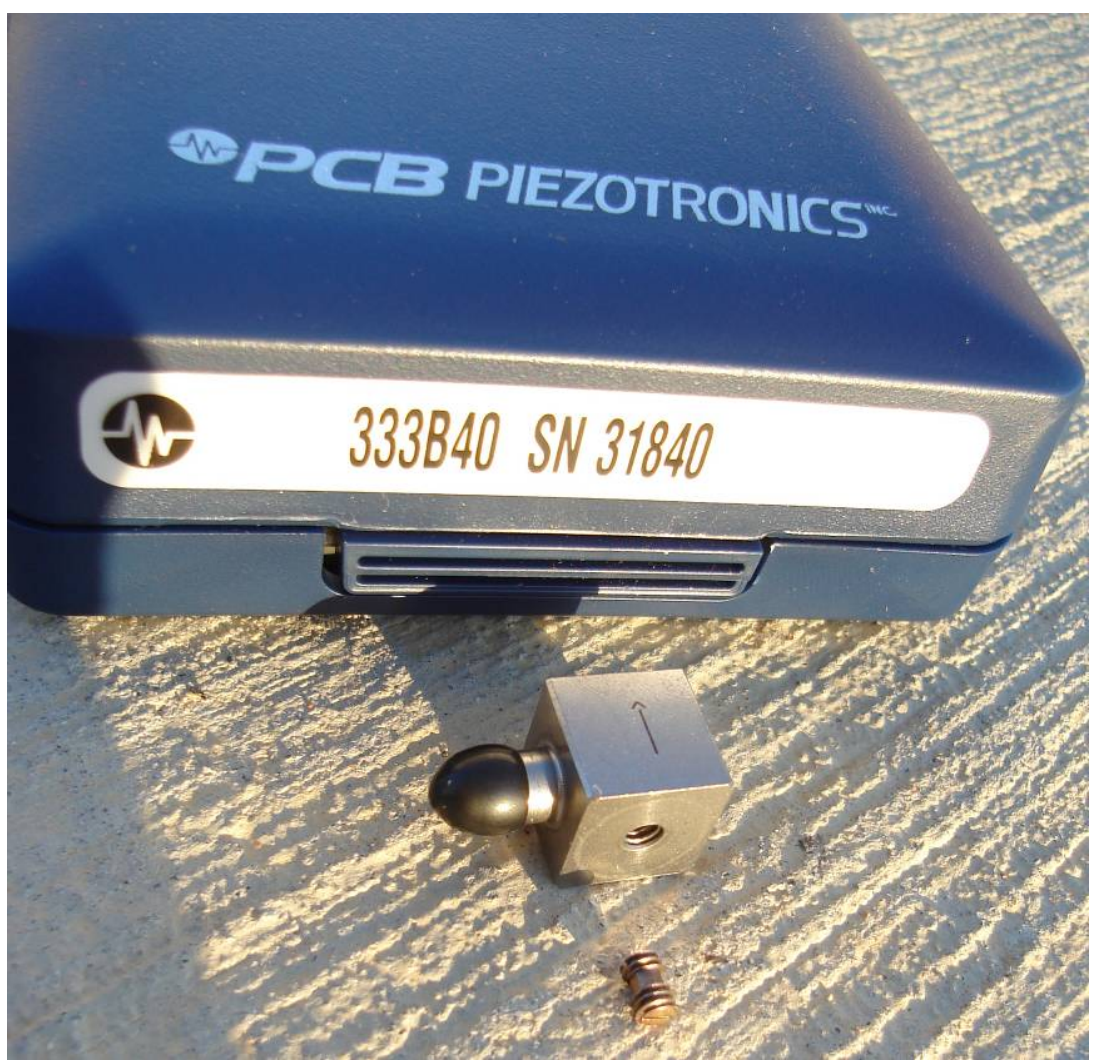

Figure 3-6 Accelerometer and Mounting Stud

\subsection{Data Collection Procedure}

The first step in the data collection procedure was to create a pan displacement signal. Using matlab's randn function, a band-limited white noise was generated. This white noise was filtered by three different shaping filters to produce three different input displacement arrays: low frequency, mixed frequency, and high frequency. The shaping filters were convolved from the filters shown in table 3-1: 
Table 3-1 Filtering Matrix

\begin{tabular}{|c|c|c|c|c|c|}
\hline \multirow{2}{*}{$\begin{array}{c}\text { Input } \\
\text { Displacement }\end{array}$} & \multicolumn{5}{|c|}{ Filters Convolved to Form Shaping Filters } \\
\cline { 2 - 6 } & 1 -pole & $\begin{array}{c}\text { 3-pole } \\
8 \mathrm{~Hz}\end{array}$ & $\begin{array}{c}\text { 4-pole } \\
80 \mathrm{~Hz}\end{array}$ & $\begin{array}{c}\text { 1-pole } \\
20 \mathrm{~Hz}\end{array}$ & $\begin{array}{c}\text { 4-pole } \\
75 \mathrm{~Hz}\end{array}$ \\
\hline Low Frequency & $\checkmark$ & $\checkmark$ & & & \\
\hline Mixed Frequency & $\checkmark$ & & $\checkmark$ & $\checkmark$ & $\checkmark$ \\
\hline High Frequency & & & & & \\
\hline
\end{tabular}

The low frequency displacement input was used in this thesis. This input displacement was transferred to the MTS controller and used to drive the actuator and quarter-car suspension. Actuator displacement and sprung and unsprung mass acceleration vs. time were recorded using the actuator LVDT and accelerometers. For compatibility with the HHT numerical integrator used for simulation, high frequency noise present in the actuator displacement signal is filtered using a 4-Pole $50 \mathrm{~Hz}$ Butterworth Filter. This filtering operation was implemented on all the data using Matlab's filtfilt function to provide zero phase distortion. Filtering the data in such a way is permissible because major suspension dynamics occur at much lower frequencies (first mode at $\sim 4 \mathrm{~Hz}$ and second mode at $\sim 28 \mathrm{~Hz}$ ) and because most of the energy, as seen in spectral plots, is below $50 \mathrm{~Hz}$.

Measured pan displacement vs. time using the low frequency input displacement is shown in Figures 3-7 and 3-8. Likewise, Figures 3-9 and 3-10 show the measured sprung and unsprung mass accelerations vs. time using the low frequency input displacement.

Several observations can be made about the experimentally-measured sprung and unsprung mass accelerations. First, it is apparent that the sprung mass acceleration has filtered high frequency acceleration content found in the unsprung mass. Likewise, the sprung mass acceleration follows the same general pattern as the unsprung mass. Both of these characteristics are expected byproducts of vehicular suspensions. It is also expected that the peak sprung mass accelerations are less than the unsprung mass accelerations. However, because this race car-derived suspension is designed for an 
environment where strict sprung mass control and quick transients are necessary, high spring and damper rates are used. These high spring and damper rates cause the peak sprung mass acceleration to be nearly the same magnitude as that of the unsprung mass. This apparent lack of sprung mass acceleration attenuation is less of an issue for race cars than for passenger cars. As seen in subsequent chapters, this "stiff" suspension contributes to it having nearly linear behavior.

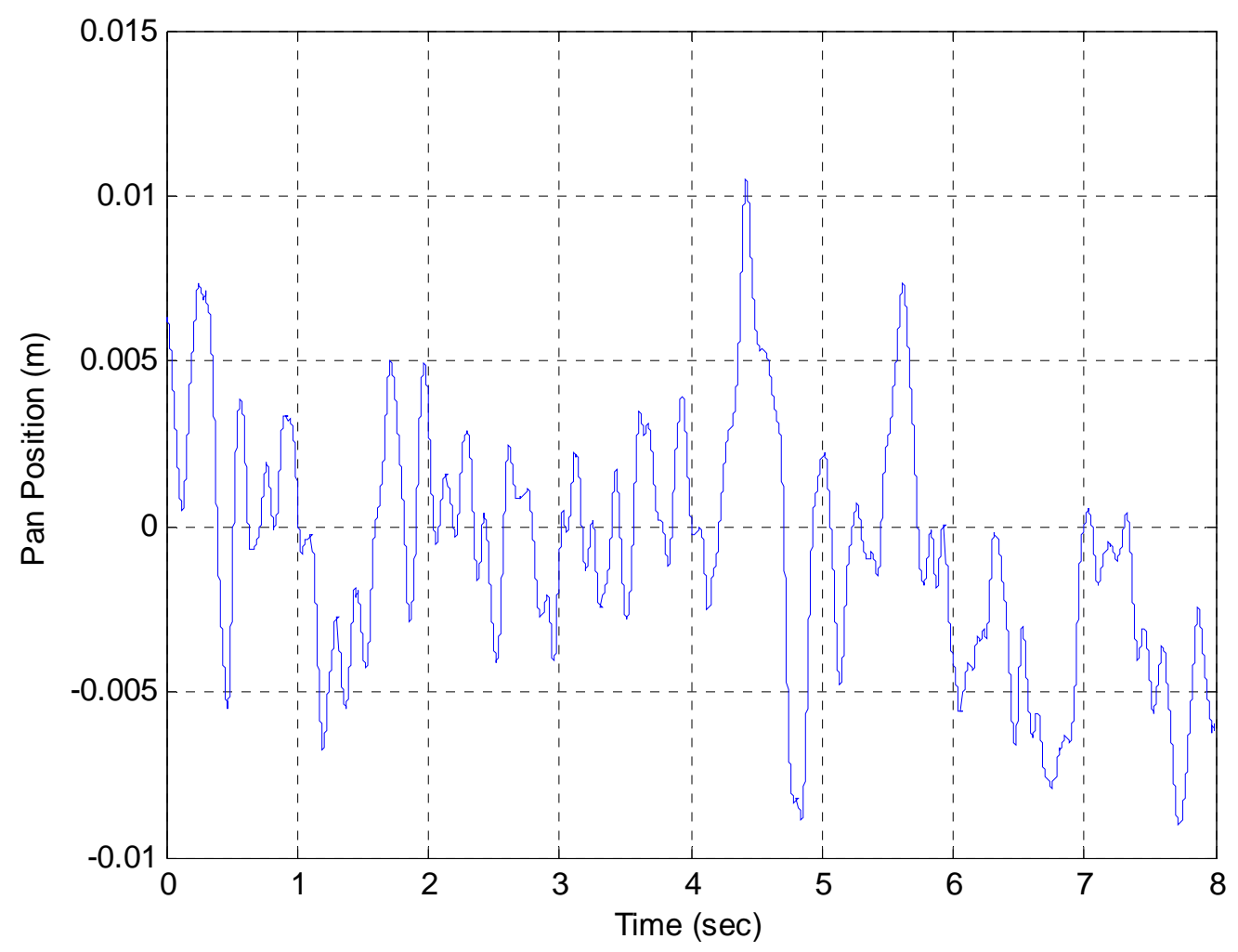

Figure 3-7 LVDT Measured Pan Position vs. Time (0-8 Sec) 


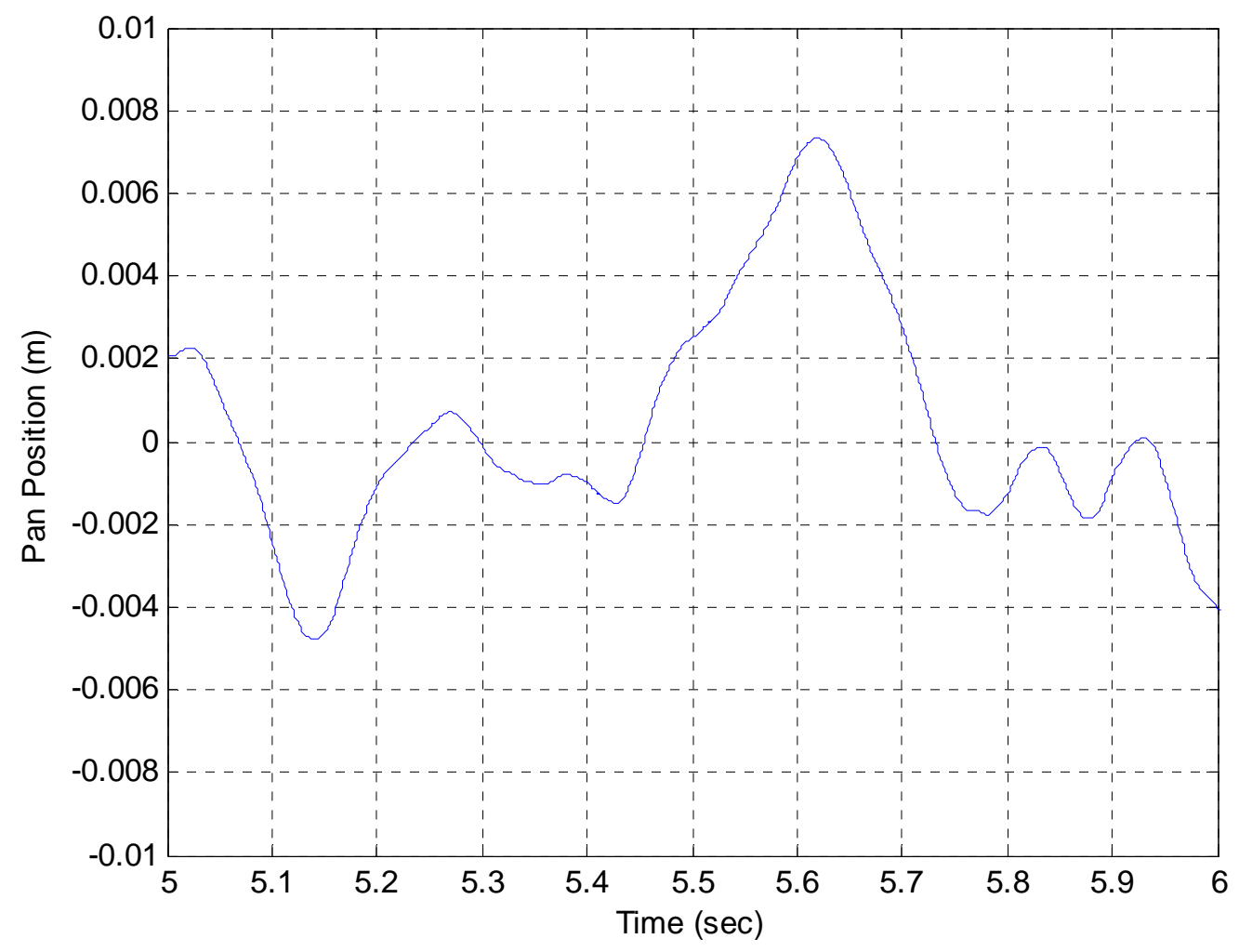

Figure 3-8 LVDT Measured Pan Position vs. Time (5-6 Sec)
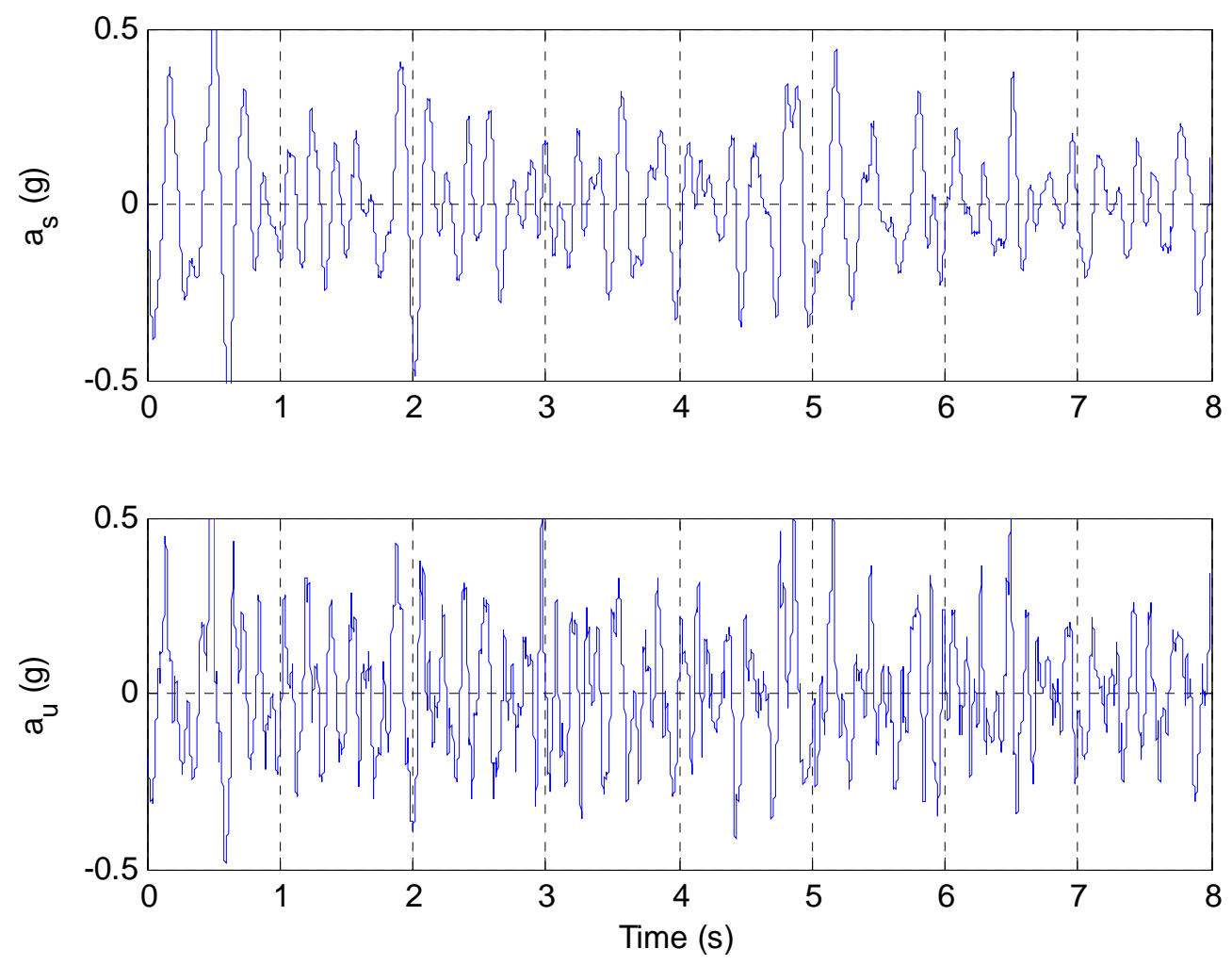

Figure 3-9 Measured Sprung and Unsprung Mass Acceleration vs. Time (0-8 Sec) 

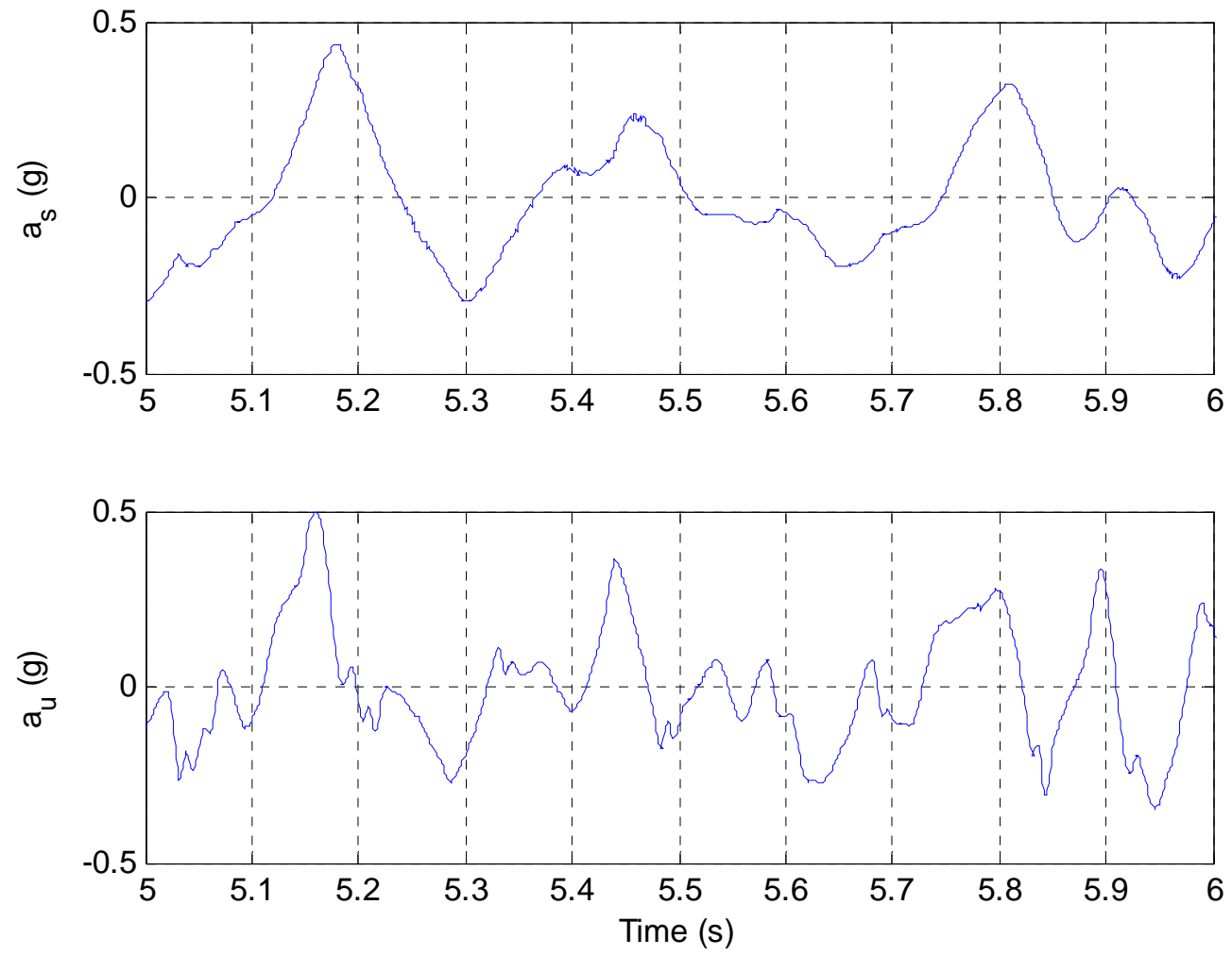

Figure 3-10 Measured Sprung and Unsprung Mass Acceleration vs. Time (5-6 Sec) 


\section{LINEAR QUARTER-CAR MODEL}

\subsection{Introduction}

This chapter develops the linear model approximation of the quarter-car McPherson strut suspension model shown in Figure 4-1. The model developed here is formulated using a multibody dynamics method as presented in [7]. This method involves solving for the differential algebraic equations of motion (DAE) that predict the linear quarter-car's dynamic response. The DAE can be written in matrix form as follows:

$$
\left[\begin{array}{cc}
\mathbf{M} & \boldsymbol{\Phi}_{\mathrm{q}}^{\mathrm{T}} \\
\mathbf{\Phi}_{\mathrm{q}} & \mathbf{0}
\end{array}\right]\left[\begin{array}{c}
\ddot{\mathbf{q}} \\
\lambda
\end{array}\right]=\left[\begin{array}{c}
\mathbf{Q}^{\mathrm{A}} \\
\alpha
\end{array}\right]
$$

where $\mathbf{M}$ is the mass matrix, $\boldsymbol{\Phi}_{\mathbf{q}}$ is the constraint Jacobian matrix, $\ddot{\mathbf{q}}$ is the generalized coordinate acceleration vector, $\boldsymbol{\lambda}$ is the vector of Lagrange multipliers, $\mathbf{Q}^{\mathbf{A}}$ is the applied force vector, and $\boldsymbol{\alpha}$ is the vector of acceleration equations.

This DAE is normally used for solving the kinematically constrained dynamic motion of rigid bodies. Because no derivatives of the Lagrange multipliers are present in this DAE it is called a mixed system of differential algebraic equations.

The motivation for using this multibody dynamics DAE approach to formulate the linear model is to allow the linear model to be simulated using the same numerical integrator used to simulate the non-linear model. Using the same numerical integrator permits comparison of the linear and non-linear models on an equal basis. Though not applicable for the linear model, an implicit benefit in formulating a dynamic model with this type of DAE is that dynamic joint reaction forces are contained in the vector of Lagrange multipliers.

To write the symbolic DAE the model's coordinates, degrees of freedom, kinematic assumptions, kinematic constraints, driving constraints, complete constraint vector, acceleration equations, applied force vector, and mass matrix must be determined. 


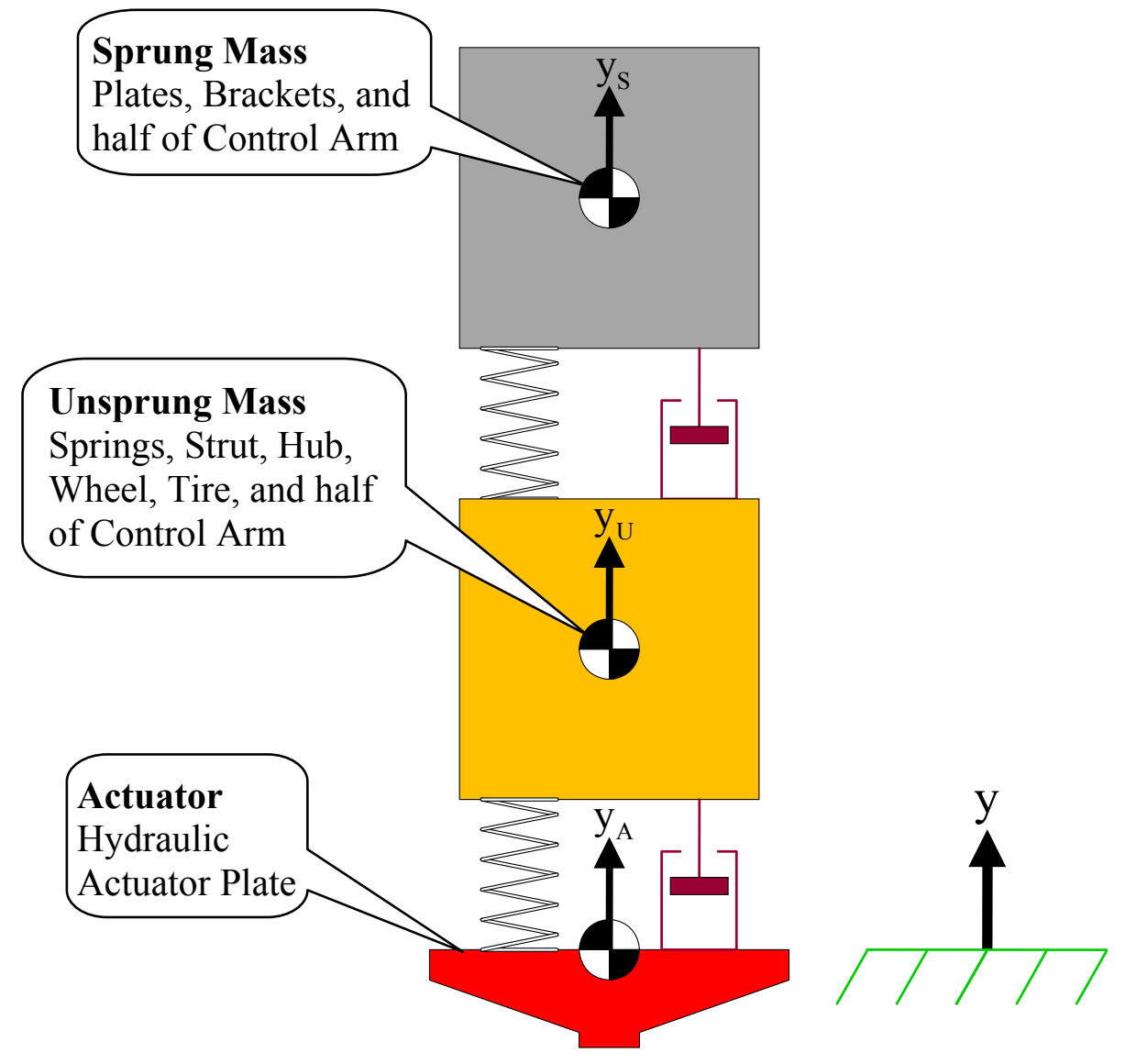

Figure 4-1 Linear Quarter-Car Model

\subsection{Coordinates}

When writing linear equations of motion it is often customary to embed coordinates at each body's CG and measure displacement from each body's equilibrium position. The linear model developed here also embeds coordinates in each body. However, for consistency with the non-linear model developed in Chapter 6, the origin of the local reference frames are positioned with respect to the global reference frame; the initial position of the actuator's top edge. 
The linear model consists of sprung, unsprung, and actuator bodies. Reference frames are embedded at the CG of the sprung and unsprung masses and at the top edge of the actuator. The global Y-position of each body is measured from its respective reference frame to the global Y reference frame. The vector of coordinates that fully define the global position of these bodies is:

$$
\mathbf{q}=\left[\begin{array}{lll}
y_{\mathrm{S}} & y_{\mathrm{U}} & y_{\mathrm{A}}
\end{array}\right]^{\mathrm{T}}
$$

Subscripts $\mathrm{S}, \mathrm{U}$, and A are abbreviations for sprung mass, unsprung mass, and actuator.

\subsection{Degrees of Freedom}

For linear systems, the degrees of freedom (DOF) present is the number of masses minus the number of driving constraints. For example, a three-mass system with one driven mass, such as the one shown in Figure 4-1, would have two DOF. Determination of the DOF in a multibody dynamics model is extended to include the constraints that joints place on the model.

The DOF present in any multibody dynamics model is the number of coordinates minus the sum of independent kinematic and driving constraint equations. Normally kinematic constraint equations are written based on how each joint constrains the motion of connected bodies. However, the linear model has no joints and therefore does not have kinematic constraint equations. Chapter 6 presents examples of how kinematic constraint equations are developed. The driven hydraulic actuator creates one driving constraint equation. In sum, there is one independent kinematic and driving constraint equation. In consideration of its three coordinates, the linear model has two DOF. One DOF is associated with the displacement of the sprung mass and the other with the unsprung mass. 


\subsection{Kinematic Assumptions and Constraints}

The major assumption made with the linear model is that the motion of the bodies in the system can be approximated as linear. In actuality the unsprung mass, and hence the strut and control arm angles, change during suspension motion. Because the linear model does not account for this angular motion the vertical motion of the sprung mass and the strut force are approximate.

Also, because the linear model has no joints it does not have kinematic constraint equations. However, implicit kinematic constraints arise by specifying only vertical coordinates. By omitting horizontal and rotational coordinates there is no need to introduce translational joints to constrain them.

\subsection{Driving Constraint}

Driving constraints prescribe the motion of driven bodies. The servo-hydraulic actuator is the driven body in this system and provides one driving constraint. In general, the driven DOF is a function of time and the model's coordinates: $y_{\mathrm{A}}=f\left(t, y_{\mathrm{S}}, y_{\mathrm{U}}\right)$. For this model the actuator position is a function only of time and can be written as the time history of its position: $y_{\mathrm{A}}=f(t)$. Thus the driving constraint is:

$$
\Phi^{\mathrm{D}}=\left[y_{\mathrm{A}}-f(t)\right]=0
$$

\subsection{Complete Constraint Vector}

The complete constraint vector is a combination of the kinematic and driving constraints:

$$
\boldsymbol{\Phi}=\left[\begin{array}{l}
\boldsymbol{\Phi}^{\mathrm{K}} \\
\boldsymbol{\Phi}^{\mathrm{D}}
\end{array}\right]=\left[\Phi^{\mathrm{D}}\right]=\left[y_{\mathrm{A}}-f(t)\right]=0
$$


where $\boldsymbol{\Phi}^{\mathrm{K}}$ is the kinematic constraint vector and $\boldsymbol{\Phi}^{\mathrm{D}}$ is the driving constraint. In this case $\boldsymbol{\Phi}^{\mathrm{K}}=[\mathbf{0}]$.

From this complete constraint vector, the Jacobian of the constraints, a matrix that plays a pivotal role in formulating the DAE, can be obtained [7]. Mathematically, the Jacobian is the partial derivative of the complete constraint vector with respect to each coordinate. Each row of the Jacobian is associated with a constraint equation. Each column of the Jacobian is associated with a coordinate. For example, an element in the third row and second column of the Jacobian is the derivative of the third constraint equation with respect to the second coordinate. In this case, since there is only one constraint equation, the Jacobian has only one row. The first column is the derivative of the constraint with respect to $y_{\mathrm{S}}$, the second column is the derivative with respect to $y_{\mathrm{U}}$, and the third column is the derivative with respect to $y_{\mathrm{A}}$. Using ' $\mathrm{i}$ ' and ' $\mathrm{j}$ ' as the row and column indices, the linear model's Jacobian is:

$$
\boldsymbol{\Phi}_{\mathrm{q}}=\frac{\partial \boldsymbol{\Phi}}{\partial \mathbf{q}}=\left[\frac{\partial \Phi_{\mathrm{i}}}{\partial q_{\mathrm{j}}}\right]_{1 \times 3}=\left[\begin{array}{lll}
\frac{\partial\left(y_{\mathrm{A}}-f(t)\right)}{\partial y_{\mathrm{S}}} & \frac{\partial\left(y_{\mathrm{A}}-f(t)\right)}{\partial y_{\mathrm{U}}} & \frac{\partial\left(y_{\mathrm{A}}-f(t)\right)}{\partial y_{\mathrm{A}}}
\end{array}\right]=\left[\begin{array}{lll}
0 & 0 & 1
\end{array}\right](4
$$

To assure that the system is not over constrained the rows of the Jacobian must be linearly independent. This Jacobian has full-row rank, and therefore the constraint equation is independent; that is, the system is not over-constrained.

\subsection{Acceleration Equations}

The acceleration equation can be obtained [7] as:

$$
\boldsymbol{\alpha}=\boldsymbol{\Phi}_{\mathrm{q}} \ddot{\mathbf{q}}=-\left(\boldsymbol{\Phi}_{\mathrm{q}} \dot{\mathbf{q}}\right)_{\mathrm{q}} \dot{\mathbf{q}}-\mathbf{2} \Phi_{\mathrm{qt}} \dot{\mathbf{q}}-\Phi_{\mathrm{tt}}
$$

The terms that make up the acceleration equation are as follows: 


$$
\begin{gathered}
\boldsymbol{\Phi}_{\mathrm{tt}}=\left[\frac{\partial^{2} \Phi_{i}}{\partial t^{2}}\right]_{n h \times 1} \\
\boldsymbol{\Phi}_{\mathrm{qt}}=\left[\frac{\partial^{2} \Phi_{i}}{\partial q_{j} \partial t}\right]_{n h \times n c} \\
\left(\boldsymbol{\Phi}_{\mathrm{q}} \dot{\mathbf{q}}\right)_{\mathrm{q}}=\left[\frac{\partial}{\partial q_{j}}\left(\sum_{k=1}^{n c} \frac{\partial \Phi_{i}}{\partial q_{k}} \dot{\mathrm{q}}_{k}\right)\right]_{n h \times n c}
\end{gathered}
$$

where 'nc' denotes the number of coordinates and 'nh' denotes the number of holonomic constraint equations. Holonomic constraint equations are those that can be expressed in terms of generalized coordinates. Nonholonomic constraint equations are more general in that they can be expressed as inequalities and may be functions of time or velocity.

It is foreseeable that a variety of driving inputs will be used to drive the quarter-car's actuator. Therefore the function $f(t)$ defining this input is kept general. Adhering to this idea, the $\boldsymbol{\Phi}_{\mathrm{tt}}$ term is:

$$
\boldsymbol{\Phi}_{\mathrm{tt}}=\left[\frac{\partial^{2} f(t)}{\partial t^{2}}\right]
$$

Because $\boldsymbol{\Phi}_{\mathrm{q}}$ has no explicitly time dependent terms, $\boldsymbol{\Phi}_{\mathrm{qt}}$ is zero. The third acceleration term is solved piecewise below:

$$
\begin{aligned}
& \boldsymbol{\Phi}_{\mathrm{q}} \dot{\mathbf{q}}=\left[\begin{array}{lll}
0 & 0 & 1
\end{array}\right]\left[\begin{array}{l}
\dot{y}_{\mathrm{S}} \\
\dot{y}_{\mathrm{U}} \\
\dot{y}_{\mathrm{A}}
\end{array}\right]=\left[\dot{y}_{\mathrm{A}}\right] \\
& \left(\boldsymbol{\Phi}_{\mathrm{q}} \dot{\mathbf{q}}\right)_{\mathrm{q}}=\left(\dot{y}_{\mathrm{A}}\right)_{\mathrm{q}}=\left[\begin{array}{lll}
0 & 0 & 0
\end{array}\right]
\end{aligned}
$$




$$
\left(\boldsymbol{\Phi}_{\mathrm{q}} \dot{\mathbf{q}}\right)_{\mathrm{q}} \dot{\mathbf{q}}=\left[\begin{array}{lll}
0 & 0 & 0
\end{array}\right]\left[\begin{array}{c}
\dot{y}_{\mathrm{S}} \\
\dot{y}_{\mathrm{U}} \\
\dot{y}_{\mathrm{A}}
\end{array}\right]=[0]
$$

Thus the acceleration equation becomes:

$$
\boldsymbol{\alpha}=-[0]-[0]-\left[\frac{d^{2} f(t)}{d t^{2}}\right]=-\frac{d^{2} f(t)}{d t^{2}}
$$

\subsection{Applied Force Vector}

This section introduces the linear model used to develop the applied force vector, $\mathbf{Q}^{\mathrm{A}}$. Figure 4-2 shows the primary spring and damper connected to the sprung and unsprung bodies and the tire spring and damper connected to the unsprung and actuator bodies. The forces applied by the springs and dampers to these bodies are shown in red. These spring and damper force elements are called translational-spring-dampers, or simply TSDs. 


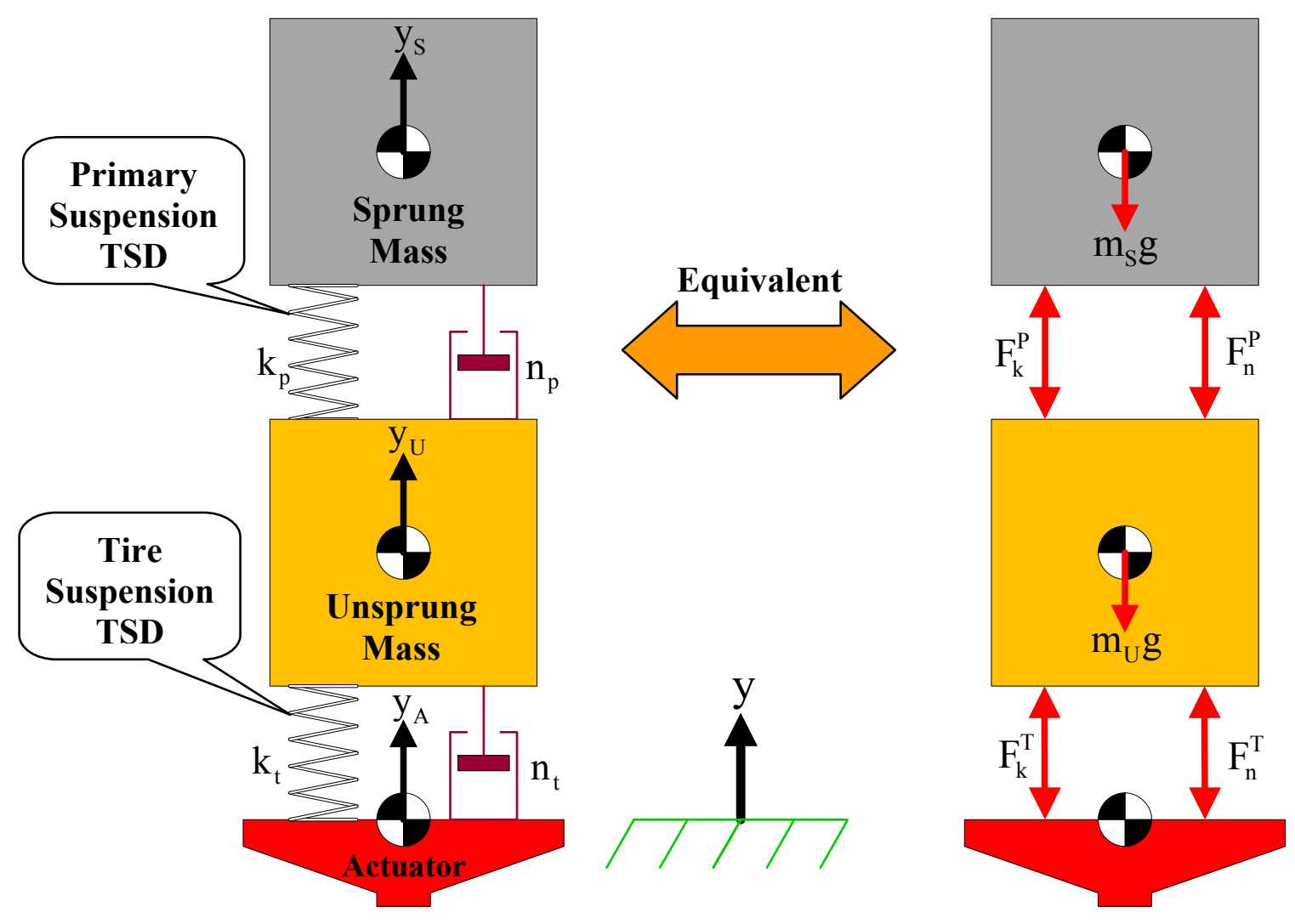

Figure 4-2 Linear Model Forces

TSDs are dynamically represented by an equal and opposite force acting on each attached body. TSD forces are collinear and act along the TSD's line of translation. The primary TSD approximates the McPherson strut's primary suspension stiffness and damping with a linear spring of stiffness $k_{p}$ and damper of damping value $n_{p}$. The tire TSD approximates the tire's stiffness and damping with a linear spring of stiffness $k_{t}$ and damper of damping value $n_{t}$.

The following sections symbolically solve for the linear model's force vector, $\mathbf{Q}^{\mathbf{A}}$. To begin with, forces corresponding to the primary and tire TSDs are developed. Next, individual force vectors are assembled from the sums of the forces acting on each body. Lastly, the complete force vector $\mathbf{Q}^{\mathrm{A}}$ is populated from each body's individual force vector. 


\subsubsection{Primary TSD}

This section develops the force created by the primary TSD. The primary TSD, shown in Figure 4-3, produces a force $\mathrm{F}^{\mathrm{P}}$ along the global $\mathrm{Y}$-direction in proportion to its compression $\Delta \mathrm{d}_{\mathrm{US}}$ and compression velocity $\Delta \dot{\mathrm{d}}_{\mathrm{US}}$.

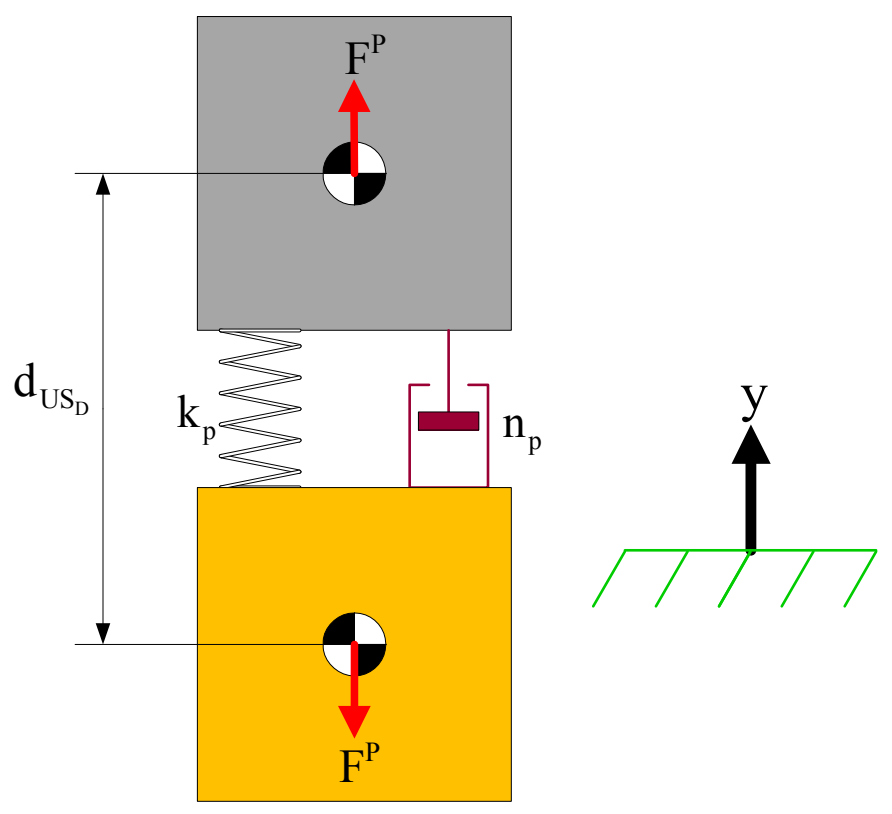

Figure 4-3 Linear Model Primary TSD

To specify the primary TSD compression, its initial and displaced length must be determined. The initial length, $\mathrm{d}_{\mathrm{US}_{\mathrm{I}}}$, is its length without any load on it, or free length. The displaced length, $\mathrm{d}_{\mathrm{US}_{\mathrm{D}}}$, is the global sprung mass Y-position minus the unsprung mass Y-position. Thus, the primary TSD compression is:

$$
\begin{aligned}
\Delta \mathrm{d}_{\mathrm{US}} & =\text { Initial length }- \text { Displaced length } \\
\Delta \mathrm{d}_{\mathrm{US}} & =\mathrm{d}_{\mathrm{US}_{\mathrm{I}}}-\mathrm{d}_{\mathrm{US}_{\mathrm{D}}} \\
\Delta \mathrm{d}_{\mathrm{US}} & =\mathrm{d}_{\mathrm{US}_{\mathrm{I}}}-\left(\mathrm{y}_{\mathrm{S}}-\mathrm{y}_{\mathrm{U}}\right)
\end{aligned}
$$


Positive spring forces are applied outward from the TSD's center towards the sprung and unsprung mass when the spring is compressed.

The first time derivative of primary TSD compression is the primary TSD compression velocity:

$$
\Delta \dot{\mathrm{d}}_{\mathrm{US}}=-\dot{\mathrm{y}}_{\mathrm{S}}+\dot{\mathrm{y}}_{\mathrm{U}}
$$

Positive damper forces are applied outward from the TSD's center towards the sprung and unsprung mass when the damper is compressing. The red vectors in Figure 4-3 show the positive primary TSD force direction. The outward primary TSD force is the sum of its spring and damper forces:

$$
\begin{aligned}
& \mathrm{F}^{\mathrm{P}}=\text { Primary Spring Force }+ \text { Primary Damper Force } \\
& \mathrm{F}^{\mathrm{P}}=\mathrm{F}_{\mathrm{k}}^{\mathrm{P}}+\mathrm{F}_{\mathrm{n}}^{\mathrm{P}} \\
& \mathrm{F}^{\mathrm{P}}=\mathrm{k}_{\mathrm{p}} \Delta \mathrm{d}_{\mathrm{US}}+\mathrm{n}_{\mathrm{p}} \Delta \dot{\mathrm{d}}_{\mathrm{US}}
\end{aligned}
$$

\subsubsection{Tire TSD}

This section develops the force created by the tire TSD. The tire TSD, shown in Figure 4-4, produces a force $\mathrm{F}^{\mathrm{T}}$ along the global $\mathrm{Y}$-direction in proportion to its compression $\Delta \mathrm{d}_{\mathrm{AU}}$ and compression velocity $\Delta \dot{\mathrm{d}}_{\mathrm{AU}}$. To specify the primary TSD compression, its initial and displaced length must be determined. The initial length, $\mathrm{d}_{\mathrm{AU}_{\mathrm{I}}}$, is its length without any load on it, or free length. The displaced length, $\mathrm{d}_{\mathrm{AU}_{\mathrm{D}}}$, is the global unsprung mass Y-position minus the actuator Y-position. Thus, the tire TSD compression is:

$$
\begin{aligned}
& \Delta \mathrm{d}_{\mathrm{AU}}=\text { Initial length }- \text { Displaced length } \\
& \Delta \mathrm{d}_{\mathrm{AU}}=\mathrm{d}_{\mathrm{AU}_{\mathrm{I}}}-\mathrm{d}_{\mathrm{AU}_{\mathrm{D}}} \\
& \Delta \mathrm{d}_{\mathrm{AU}}=\mathrm{d}_{\mathrm{AU}_{\mathrm{I}}}-\left(\mathrm{y}_{\mathrm{U}}-\mathrm{y}_{\mathrm{A}}\right)
\end{aligned}
$$




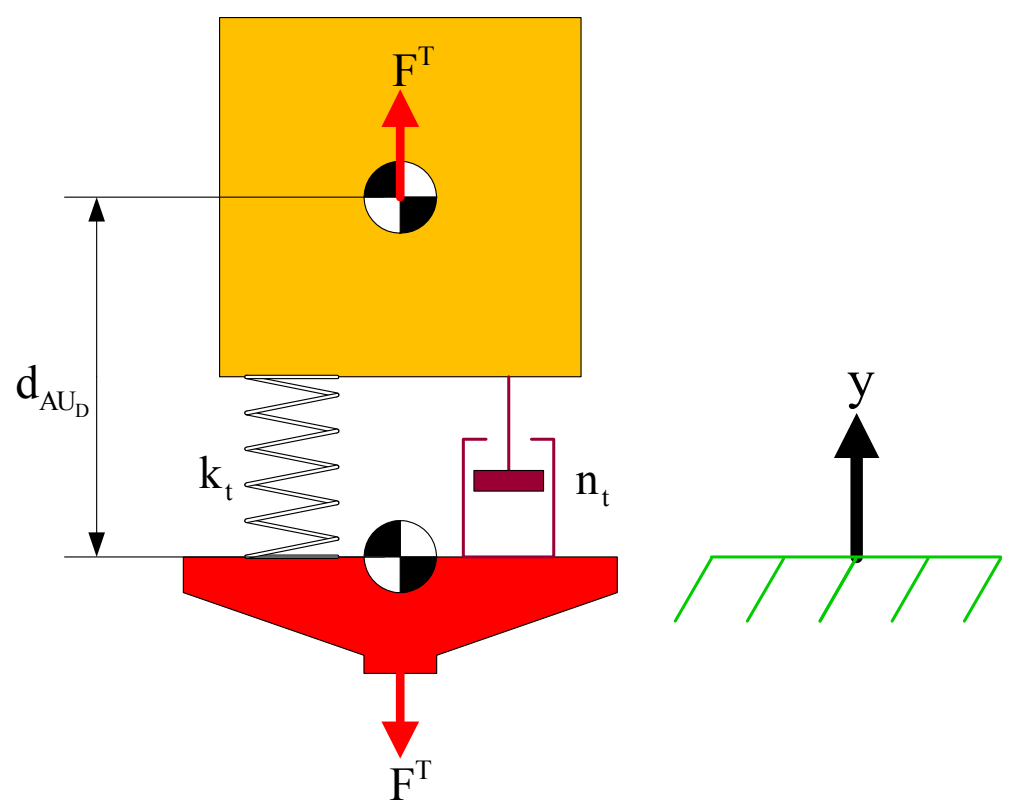

Figure 4-4 Linear Model Tire TSD

Positive spring forces are applied outward from the TSD's center towards the unsprung mass and actuator when the spring is compressed.

The first time derivative of tire TSD compression is the tire TSD compression velocity:

$$
\Delta \dot{\mathrm{d}}_{\mathrm{AU}}=-\dot{\mathrm{y}}_{\mathrm{U}}+\dot{\mathrm{y}}_{\mathrm{A}}
$$

Positive damper forces are applied outward from the TSD's center towards the unsprung mass and actuator when the damper is compressing. The red vectors in Figure 4-4 show the positive tire TSD force direction. The outward tire TSD force is the sum of its spring and damper forces:

$$
\begin{aligned}
& \mathrm{F}^{\mathrm{T}}=\text { Tire Spring Force }+ \text { Tire Damper Force } \\
& \mathrm{F}^{\mathrm{T}}=\mathrm{F}_{\mathrm{k}}^{\mathrm{T}}+\mathrm{F}_{\mathrm{n}}^{\mathrm{T}} \\
& \mathrm{F}^{\mathrm{T}}=\mathrm{k}_{\mathrm{t}} \Delta \mathrm{d}_{\mathrm{AU}}+\mathrm{n}_{\mathrm{t}} \Delta \dot{\mathrm{d}}_{\mathrm{AU}}
\end{aligned}
$$




\subsubsection{Sprung Mass Forces}

This section identifies the applied sprung mass forces. The forces acting on this body are due to the primary TSD and gravity. These forces act at the CG as shown in Figure 4-5.
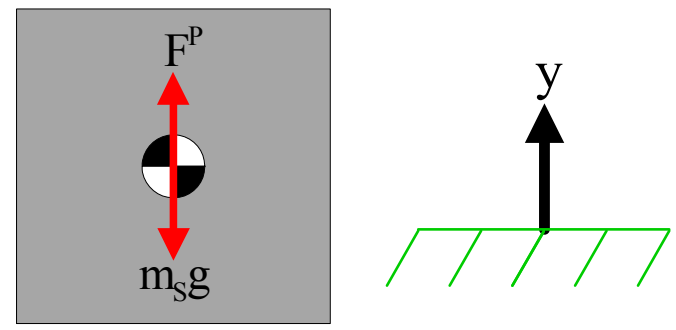

\section{Figure 4-5 Linear Model Sprung Mass Forces}

The sprung mass and gravitational acceleration are $\mathrm{m}_{\mathrm{S}}$ and $\mathrm{g}$, respectively. With these variables defined, the sum of forces applied to the sprung mass in the global Y-direction is:

$$
\sum \mathrm{F}_{\mathrm{Sy} \uparrow}^{\mathrm{A}}=\mathrm{F}^{\mathrm{P}}-\mathrm{m}_{\mathrm{s}} \mathrm{g}
$$

where $\mathrm{F}^{\mathrm{P}}$ has been derived in Section 4.8.1.

\subsubsection{Unsprung Mass Forces}

This section identifies the applied unsprung mass forces. The forces acting on this body are due to the primary and tire TSDs and gravity. These forces act at the CG as shown in Figure 4-6.
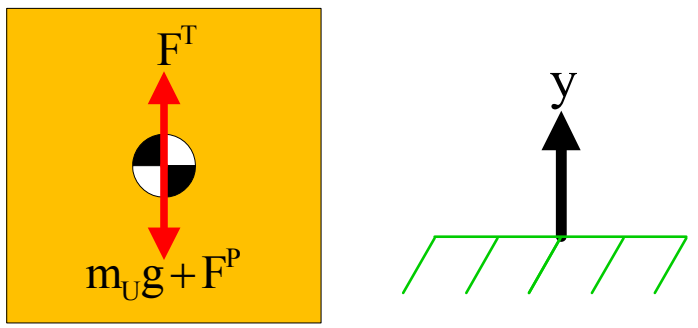

Figure 4-6 Linear Model Unsprung Mass Forces 
The unsprung mass and gravitational acceleration are $\mathrm{m}_{\mathrm{U}}$ and $\mathrm{g}$, respectively. With these variables defined, the sum of forces applied to the unsprung mass in the global $\mathrm{Y}$ direction is:

$$
\sum \mathrm{F}_{\mathrm{Uy} \uparrow}^{\mathrm{A}}=\mathrm{F}^{\mathrm{T}}-\mathrm{F}^{\mathrm{P}}-\mathrm{m}_{\mathrm{U}} \mathrm{g}
$$

where $\mathrm{F}^{\mathrm{P}}$ has been derived in Section 4.8.1 and $\mathrm{F}^{\mathrm{T}}$ in Section 4.8.2.

\subsubsection{Actuator Forces}

The servo-hydraulic actuator accounts for the force acting on it and inputs a precisely controlled wheel pan position time history to the quarter-car rig. Because the actuator's controller processes the dynamics associated with this force it is set to zero:

$$
\sum \mathrm{F}_{\mathrm{Ay} \uparrow}^{\mathrm{A}}=0
$$

\subsubsection{Complete Force Vector}

The complete force vector $\mathbf{Q}^{\mathbf{A}}$ is populated from each body's individual force vector:

$$
\mathbf{Q}^{\mathrm{A}}=\left[\begin{array}{c}
\sum \mathrm{F}_{\mathrm{Sy} \uparrow}^{\mathrm{A}} \\
\sum \mathrm{F}_{\mathrm{Uy} \uparrow}^{\mathrm{A}} \\
\sum \mathrm{F}_{\mathrm{Ay} \uparrow}^{\mathrm{A}}
\end{array}\right]=\left[\begin{array}{c}
\mathrm{F}^{\mathrm{P}}-\mathrm{m}_{\mathrm{S}} \mathrm{g} \\
\mathrm{F}^{\mathrm{T}}-\mathrm{F}^{\mathrm{P}}-\mathrm{m}_{\mathrm{U}} \mathrm{g} \\
0
\end{array}\right]
$$




\subsection{Mass Matrix}

The mass matrix for the linear model has the form:

$$
\mathbf{M}=\left[\begin{array}{ccc}
\mathrm{m}_{\mathrm{S}} & 0 & 0 \\
0 & \mathrm{~m}_{\mathrm{U}} & 0 \\
0 & 0 & \mathrm{~m}_{\mathrm{A}}
\end{array}\right]
$$

where $\mathrm{m}_{\mathrm{S}}, \mathrm{m}_{\mathrm{U}}$, and $\mathrm{m}_{\mathrm{A}}$ are the sprung, unsprung, and actuator masses. The sprung mass includes mass from the sprung mass plate, adapter plate, strut tower, and ballast weights. The unsprung mass includes mass from the springs, damper, upright, brake disk, wheel, and tire. Tie-rod and control arm masses are split evenly between the sprung and unsprung masses. The actuator mass is considered negligible in this study and is approximated as zero. For completeness and for easy adjustment of the model in the future, the dynamic equation associated with $\mathrm{m}_{\mathrm{A}}$ is kept in the system of equations.

\subsection{McPherson Strut Linear DAE}

With all the components of the DAE determined, it can be assembled. The DAE used to simulate the linear quarter-car's dynamic response is:

$$
\begin{aligned}
& {\left[\begin{array}{cc}
\mathbf{M} & \boldsymbol{\Phi}_{\mathbf{q}}^{\mathbf{T}} \\
\boldsymbol{\Phi}_{\mathbf{q}} & \mathbf{0}
\end{array}\right]\left[\begin{array}{c}
\ddot{\mathbf{q}} \\
\boldsymbol{\lambda}
\end{array}\right]=\left[\begin{array}{c}
\mathbf{Q}^{\mathbf{A}} \\
\boldsymbol{\alpha}
\end{array}\right]} \\
& {\left[\begin{array}{cccc}
\mathrm{m}_{\mathrm{S}} & 0 & 0 & 0 \\
0 & \mathrm{~m}_{\mathrm{U}} & 0 & 0 \\
0 & 0 & \mathrm{~m}_{\mathrm{A}} & 1 \\
0 & 0 & 1 & 0
\end{array}\right]\left[\begin{array}{c}
\ddot{y}_{\mathrm{S}} \\
\ddot{y}_{\mathrm{U}} \\
\ddot{y}_{\mathrm{A}} \\
\lambda_{1}
\end{array}\right]=\left[\begin{array}{c}
\mathrm{F}^{\mathrm{P}}-\mathrm{m}_{\mathrm{S}} \mathrm{g} \\
\mathrm{F}^{\mathrm{T}}-\mathrm{F}^{\mathrm{P}}-\mathrm{m}_{\mathrm{U}} \mathrm{g} \\
0 \\
-\frac{d^{2} f(t)}{d t^{2}}
\end{array}\right]}
\end{aligned}
$$




\section{LINEAR QUARTER-CAR MODEL SYSTEM IDENTIFICATION}

\subsection{Introduction}

This chapter presents the background and results of the system identification of the linear quarter-car McPherson strut suspension model developed in Chapter 4. The purpose of this system identification is to determine model parameters that best facilitate the linear model's prediction of the experimental sprung and unsprung mass accelerations. Using these parameters with the linear model will create an optimal linear model that can be used for dynamic response prediction and control algorithm design.

\subsection{Hilber-Hughes-Taylor DAE Integrator}

A Hilber-Hughes-Taylor (HHT) method integrator was used to solve the system of DAEs. In addition to being capable of solving DAE equations this method is attractive because of its intrinsic stability. Negrut [13] mentions that "models that are mechanically stiff [can land other integrators] outside the stability region [but this] is a non-issue with the HHT method, which is a fixed low-order method with good stability properties in the whole left plane." The basic fixed time step HHT algorithm employed in this study is as follows:

1) Set initial conditions and parameters

2) Calculate initial acceleration

3) Enter main-loop

a. Calculate values that remain constant during each time step

i. Enter sub-loop

1. Calculate force and kinematic residuals

2. From residuals solve for delta vector

3. Add delta vector to state vector

ii. End sub-loop when residuals and delta are less than epsilon

b. Record state vector

4) End main-loop when all time steps have been solved 
The state vector refers to the vector of positions, velocities, accelerations, and Lagrange multipliers. Residuals are calculated based on the current state vector. The sub-loop uses multiple function evaluations to predict the state vector that minimizes the residuals, and thus this method is closer to a predictor-corrector method than a Euler method. The subloop terminates when the norm of the residuals and the norm of the delta vector are less than epsilon. In this way the HHT algorithm provides a user-specified error tolerance. For this study, epsilon was chosen to be $\varepsilon=10^{-4}$ because this value allowed timely simulation and because the simulation was not significantly more accurate with a lower epsilon.

Another value that must be specified with this method is alpha. From an implementation standpoint, a lower alpha value corresponds to a shorter simulation transient period. Alpha was chosen to be the minimum allowable for this method $(\alpha=-1 / 3)$ to shorten the initial simulation transient. With these constants specified, the HHT integrator was used to solve the DAE system and served as the basic algorithm to which system identification was applied.

\subsection{System Identification}

System identification was performed by minimizing the acceleration error function:

$$
\begin{aligned}
& e_{\mathrm{S}}(\mathrm{i})=\operatorname{Exp} \_\mathrm{a}_{\mathrm{S}}(\mathrm{i})-\operatorname{Sim} \_\mathrm{a}_{\mathrm{S}}(\mathrm{i}) \\
& e_{\mathrm{U}}(\mathrm{i})=\operatorname{Exp} \_\mathrm{a}_{\mathrm{U}}(\mathrm{i})-\operatorname{Sim} \_\mathrm{a}_{\mathrm{U}}(\mathrm{i}) \\
& \mathrm{f}_{\mathrm{S}}=\sum_{1}^{n} e_{\mathrm{S}}(\mathrm{i}) \cdot e_{\mathrm{S}}(\mathrm{i}) \\
& \mathrm{f}_{\mathrm{U}}=\sum_{1}^{n} e_{\mathrm{U}}(\mathrm{i}) \cdot e_{\mathrm{U}}(\mathrm{i}) \\
& \mathrm{f}=\mathrm{f}_{\mathrm{S}}+\mathrm{f}_{\mathrm{U}}
\end{aligned}
$$

where $\operatorname{Exp} \__{\mathrm{S}}$ and $\operatorname{Exp} \_\mathrm{a}_{U}$ are the experimental sprung and unsprung mass acceleration arrays, $\operatorname{Sim} \_\mathrm{a}_{\mathrm{S}}(\mathrm{i})$ and $\operatorname{Sim} \_\mathrm{a}_{\mathrm{U}}(\mathrm{i})$ are the simulated sprung and unsprung mass 
acceleration arrays, $e_{\mathrm{S}}$ and $e_{\mathrm{U}}$ are the sprung and unsprung mass acceleration error arrays, $f_{S}$ and $f_{U}$ are the sum-of-squared sprung and unsprung mass acceleration error scalars, and $\mathrm{f}$ is the total acceleration error function. In finding a minimum to this error function, also called the cost function, the system identification process finds optimal parameter values. In this case, optimal parameters are defined as parameters that minimize the difference between the simulated and experimental sprung and unsprung mass accelerations. Using these optimized parameters with the linear quarter-car model allows it to best predict the sprung and unsprung mass accelerations.

\subsection{Procedure}

This section describes the method used to perform system identification. To perform system identification, one selects the period over which the cost function is evaluated, the parameters to be optimized, and the upper and lower parameter bounds.

The comparison period used to generate the cost function was 3-8 seconds. This interval was chosen because it allows sufficient time for the simulation to pass its transient, yet it is short enough to ensure that the system identification time was not excessive.

The optimization parameters selected for linear model system identification include the sprung mass, unsprung mass, the stiffness values, and damping values. Initial values for these parameters were gathered by direct measurement, calculation, or estimation. Figure 5-1 shows the initial parameter estimates that comprise the non-optimized parameters. Figure 5-2 shows the initial positions and velocities.

The choice of parameter bounds directly affects the quantity of separate system identification runs needed to converge to optimal parameters. If the system identification process arrives at a parameter equal to that parameter's bound, it may be the optimal parameter or it may be the best parameter available based on the bound limitation set. In order to find out which is the case, this parameter's bound must be expanded and subsequent system identification performed. Subsequent system identification uses the 
optimal parameters solved for in the previous system identification. In the interest of avoiding the system identification process solving for optimal parameters at its bounds, and thus having to perform subsequent system identification, the initial bounds are broadly spaced. If subsequent system identification is needed, the bounds are less broadly spaced. Further system identification is not run if none of the optimized parameters are at a bound or if extending the bound has minimal effect on the model's performance. 


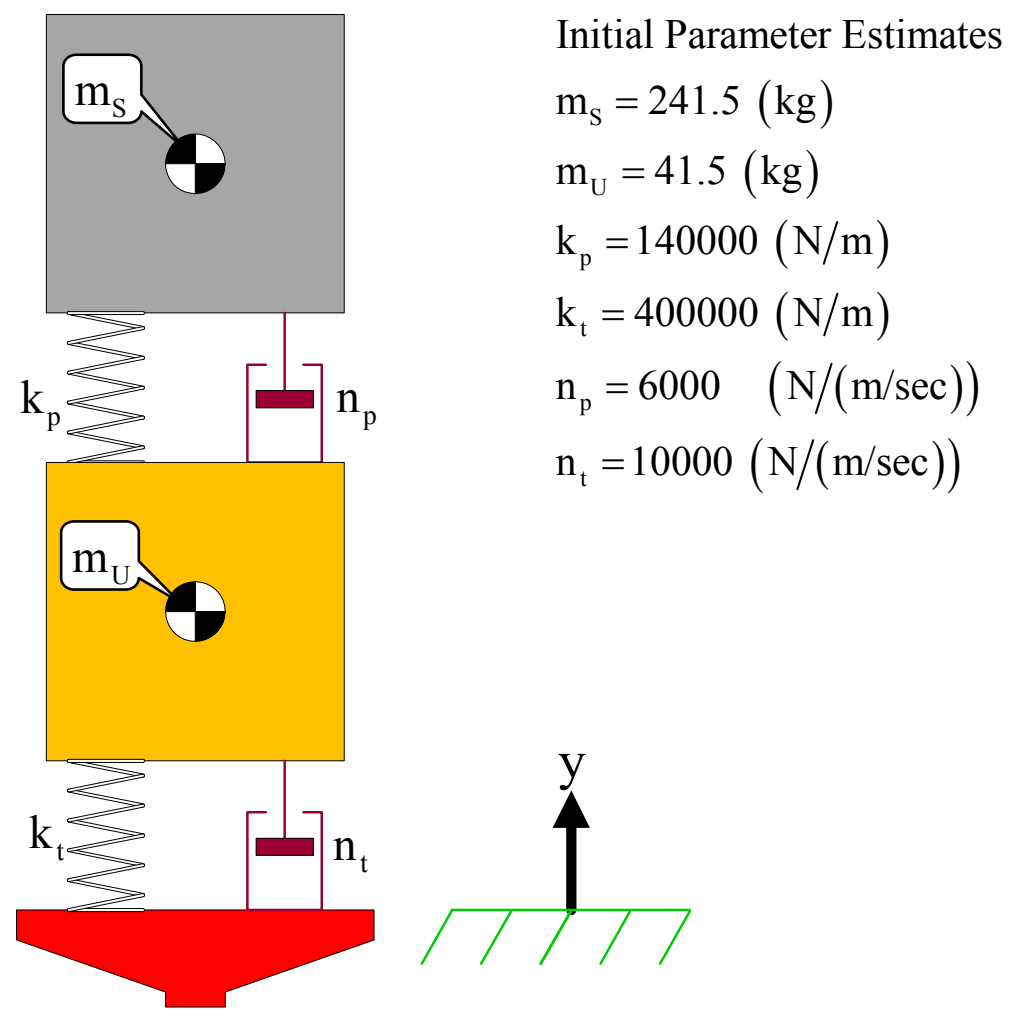

Figure 5-1 Linear Model Initial Parameter Estimates

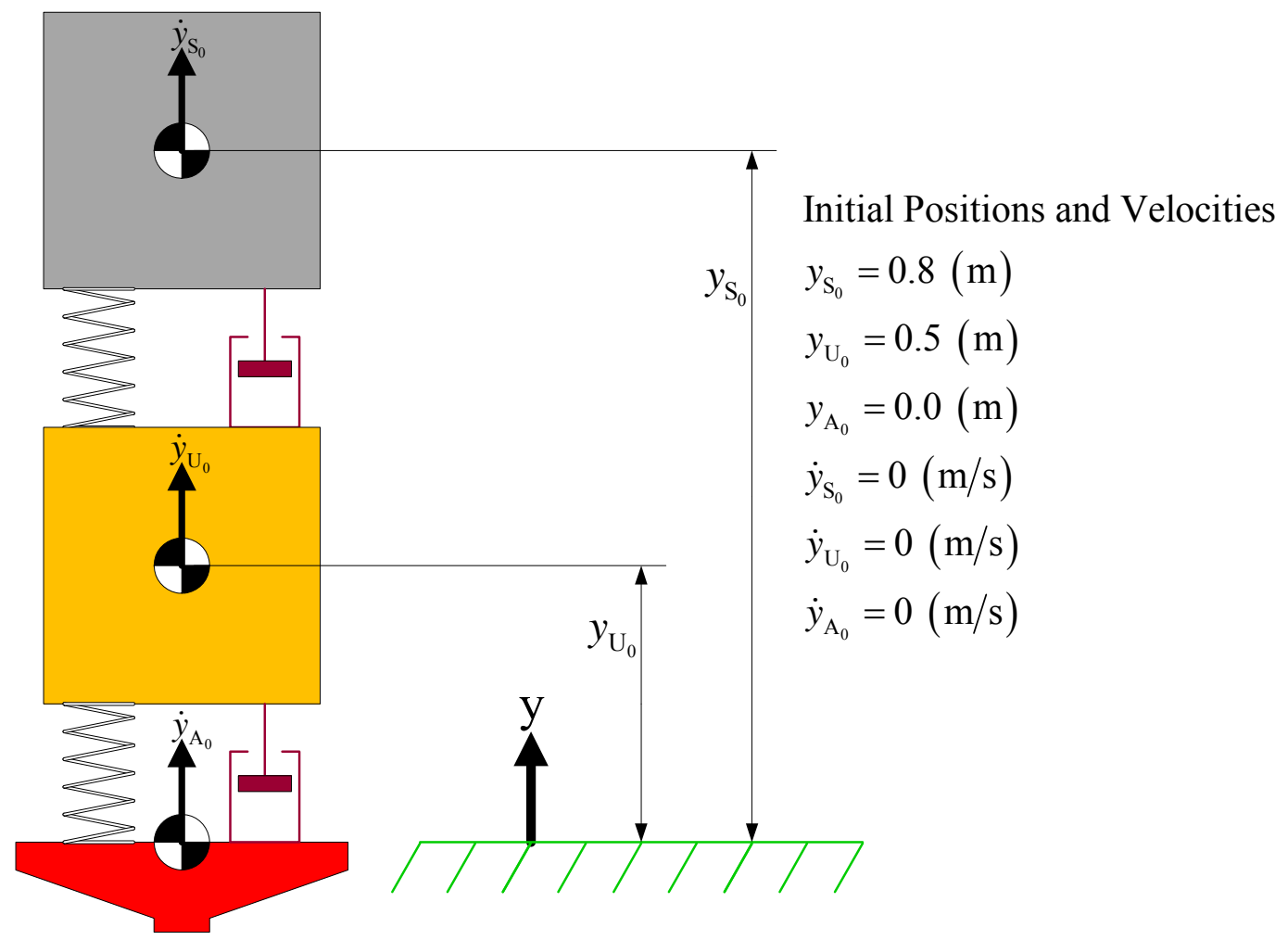

Figure 5-2 Linear Model Initial Conditions 


\subsection{Performance Measure}

The performance metric chosen to judge how well the mathematical model predicts the experimental acceleration is the ratio of the root-mean-square (RMS) acceleration error to the experimental RMS acceleration over the 3 to 50 second time interval. These ratios, expressed in decibel form, for the sprung and unsprung mass are:

$$
\begin{aligned}
& \mathrm{R}_{\mathrm{S}}=20 \log 10\left(\frac{\operatorname{RMS}\left(e_{\mathrm{S}}\right)}{\operatorname{RMS}\left(\operatorname{Exp} \mathrm{a}_{\mathrm{S}}\right)}\right) \\
& \mathrm{R}_{\mathrm{U}}=20 \log 10\left(\frac{\operatorname{RMS}\left(e_{\mathrm{U}}\right)}{\operatorname{RMS}\left(\operatorname{Exp} \mathrm{a}_{\mathrm{U}}\right)}\right)
\end{aligned}
$$

where $R_{S}$ and $R_{U}$ are the sprung and unsprung mass performance ratios. These ratios, rather than the absolute errors, were chosen as performance criteria because they are nondimensional numbers that can be interpreted as a ratio of signal power: the lower the ratio, the better the performance. Also, these numbers are portable in the sense that they can be used to compare both the linear and non-linear models' performance.

\subsection{Linear System Identification Results}

Figure 5-3 compares the acceleration predicted by the mathematical model (green dashed) using the initial parameter estimates with the experimentally measured accelerations (blue - solid). The error is highlighted in red (dash - dot). This nonoptimized model produces sprung and unsprung mass performance ratios of:

$$
\begin{aligned}
& R_{S}=-10.444 d B \\
& R_{U}=-2.973 d B
\end{aligned}
$$


Figure 5-4 compares the acceleration predicted by the mathematical model using optimized parameters with the experimentally measured accelerations. This optimized model produces sprung and unsprung mass performance ratios of:

$$
\begin{aligned}
& R_{S}=-18.257 \mathrm{~dB} \\
& R_{U}=-8.2199 \mathrm{~dB}
\end{aligned}
$$

Table 5-1 shows the optimal parameters for the linear model:

Table 5-1 Linear Model Optimal Parameters

\begin{tabular}{|c|c|c|c|c|c|c|}
\hline & $\mathbf{m s}$ & $\mathbf{k p}$ & $\mathbf{n p}$ & $\mathbf{m u}$ & $\mathbf{k t}$ & $\mathbf{n t}$ \\
\hline Opt & 205.258 & 151380 & 5437.9 & 142.679 & 396040 & 7899.9 \\
\hline
\end{tabular}
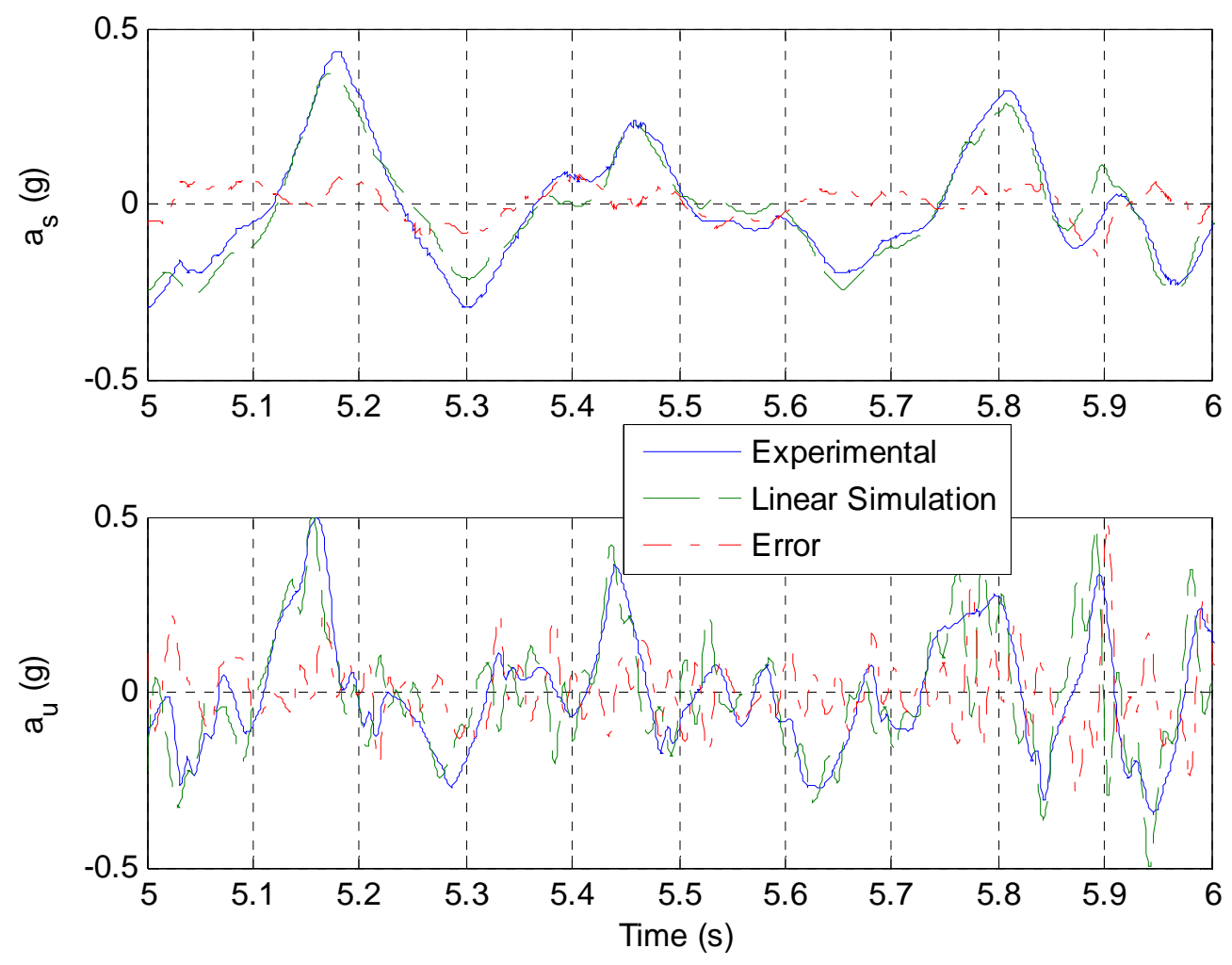

Figure 5-3 Experimental vs. Simulated Linear Model Acceleration with NonOptimized Parameters 

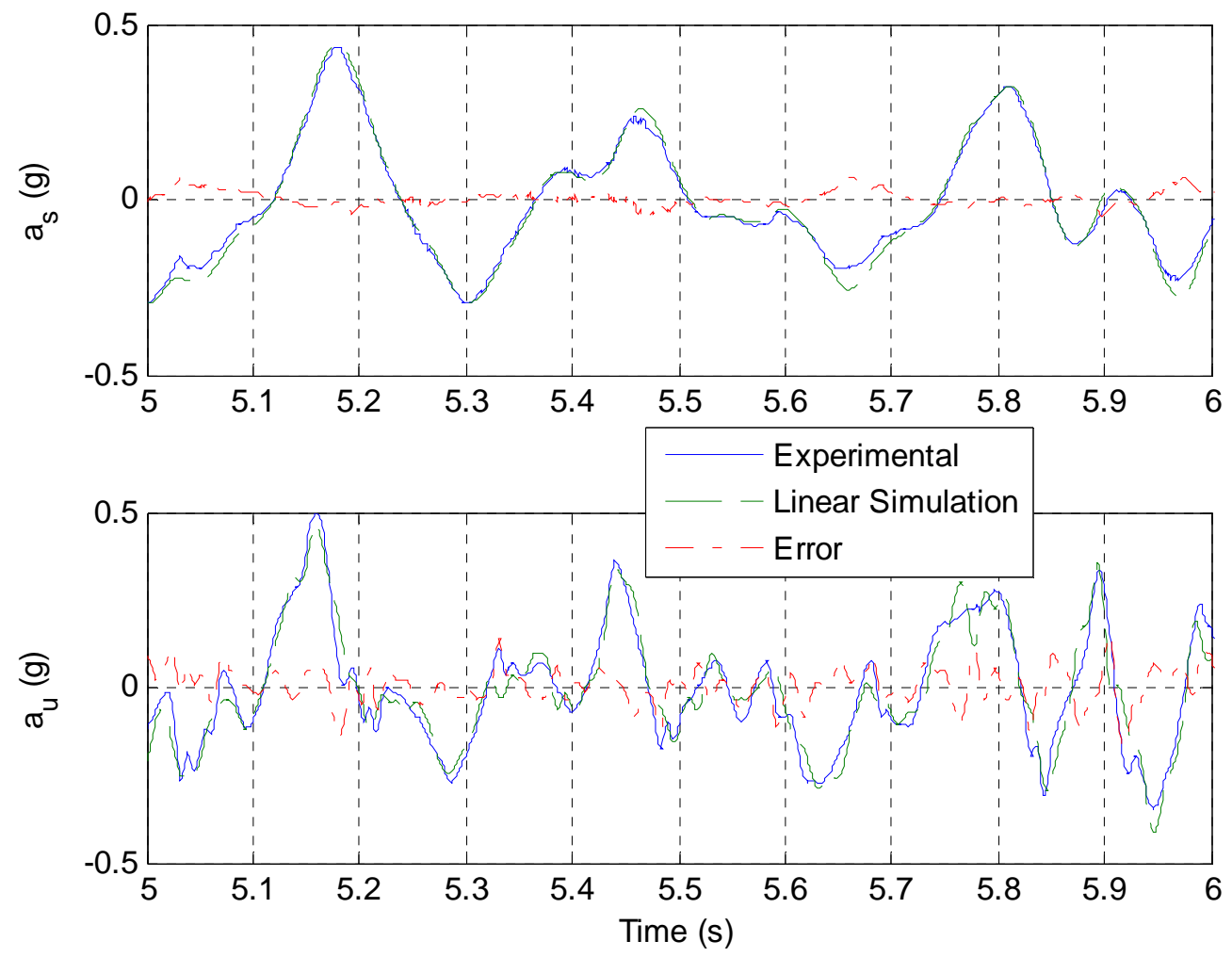

Figure 5-4 Experimental vs. Simulated Linear Model Acceleration with Optimized Parameters 


\section{NON-LINEAR QUARTER-CAR MODEL}

\subsection{Introduction}

This chapter develops the multibody dynamics quarter-car McPherson strut suspension model shown in Figure 6-1. Differential algebraic equations of motion (DAE) that predict the quarter-car's dynamic response are assembled in the following symbolic matrix form:

$$
\left[\begin{array}{cc}
\mathbf{M} & \mathbf{\Phi}_{\mathrm{q}}^{\mathrm{T}} \\
\mathbf{\Phi}_{\mathrm{q}} & \mathbf{0}
\end{array}\right]\left[\begin{array}{c}
\ddot{\mathbf{q}} \\
\lambda
\end{array}\right]=\left[\begin{array}{c}
\mathbf{Q}^{\mathrm{A}} \\
\boldsymbol{\alpha}
\end{array}\right]
$$

\subsection{Coordinates}

This model consists of sprung mass, unsprung mass, control arm, and actuator bodies. The sprung mass, unsprung mass, and control arm positions are specified by their global $\mathrm{X}-\mathrm{Y}$ CG position and orientation angle. Because its horizontal reaction force is of no interest the actuator is specified by only the global Y-position of its top edge. The vector of coordinates that define the global position and orientation of these bodies is:

$$
\mathbf{q}=\left[\begin{array}{llllllllll}
x_{\mathrm{S}} & y_{\mathrm{S}} & \phi_{\mathrm{S}} & x_{\mathrm{C}} & y_{\mathrm{C}} & \phi_{\mathrm{C}} & x_{\mathrm{U}} & y_{\mathrm{U}} & \phi_{\mathrm{U}} & y_{\mathrm{A}}
\end{array}\right]^{\mathrm{T}}
$$

Subscripts S, U, C, and A are abbreviations for sprung mass, unsprung mass, control arm, and actuator.

Local coordinates are denoted with a prime. The sprung mass, unsprung mass, and control arm local coordinate axes originate from these bodies' respective CGs. Orientation angles are measured between the global $\mathrm{X}$-axis and local $\mathrm{x}$-axes. The local $\mathrm{x}$ axis directions correspond to body-fixed references. In particular, $x_{\mathrm{S}}^{\prime}, x_{\mathrm{U}}^{\prime}$, and $x_{\mathrm{C}}^{\prime}$ correspond to the sprung mass's horizontal edge, the line perpendicular to the strut's revolute joints, and the line parallel to the control arm's revolute joints. 


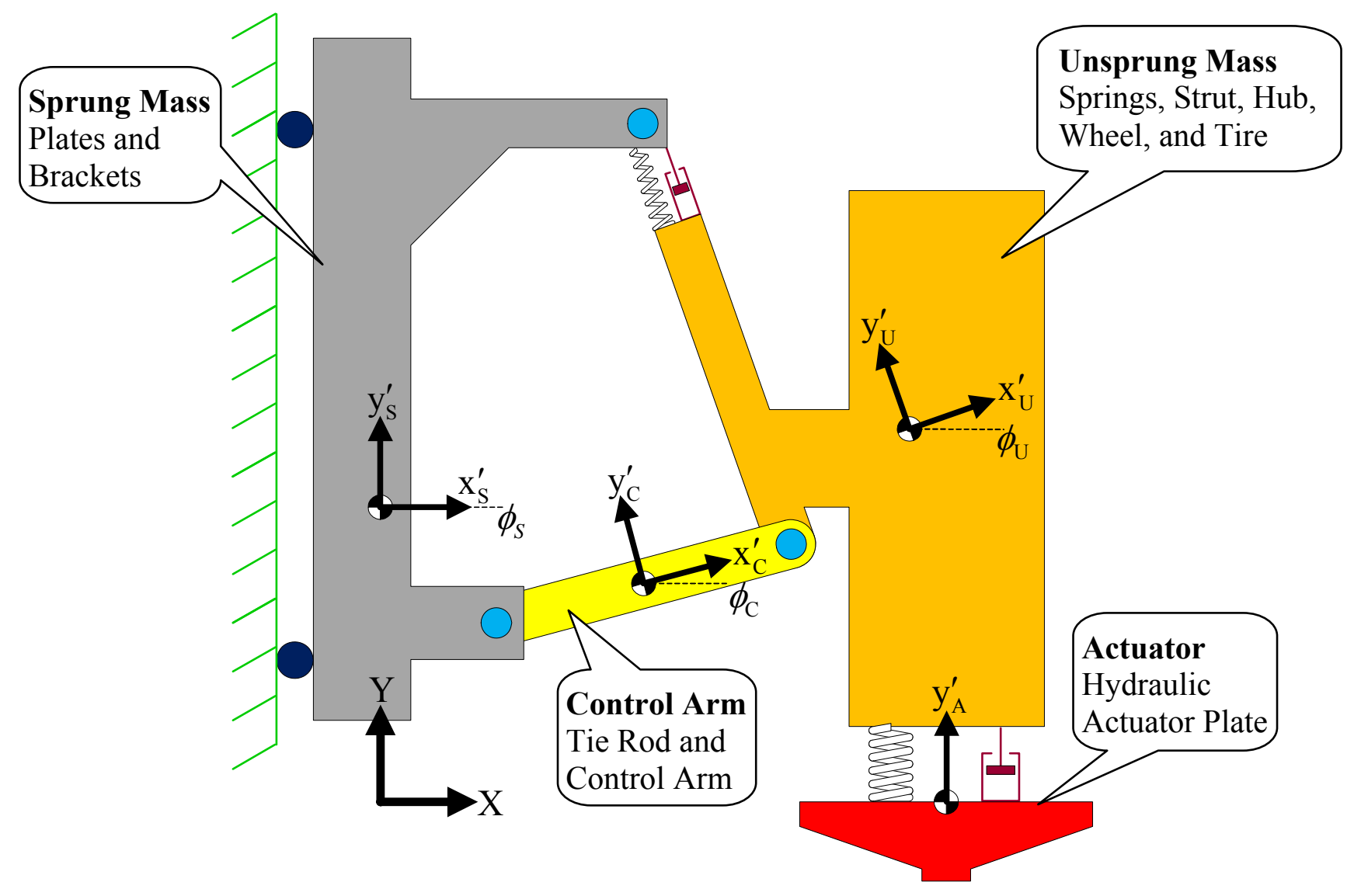

Figure 6-1 Non-Linear Quarter-Car Model

\subsection{Degrees of Freedom}

The degrees of freedom (DOF) present in this multibody dynamics model is its number of coordinates minus its number of independent constraint equations. Figure 6-2 shows the joints from which kinematic constraint equations are written (see details in subsequent sections). The revolute-translational composite joint produces one constraint (restricts one translational DOF). Each of this model's two revolute joints produces two constraints (each restricts two translational DOF). Likewise, the translational joint produces two constraints (restricts one translational and one rotational DOF). The driven hydraulic actuator creates one driving constraint equation. In sum, there are eight independent kinematic and driving constraint equations. Thus, in consideration of its ten coordinates, the non-linear model has two DOF. 


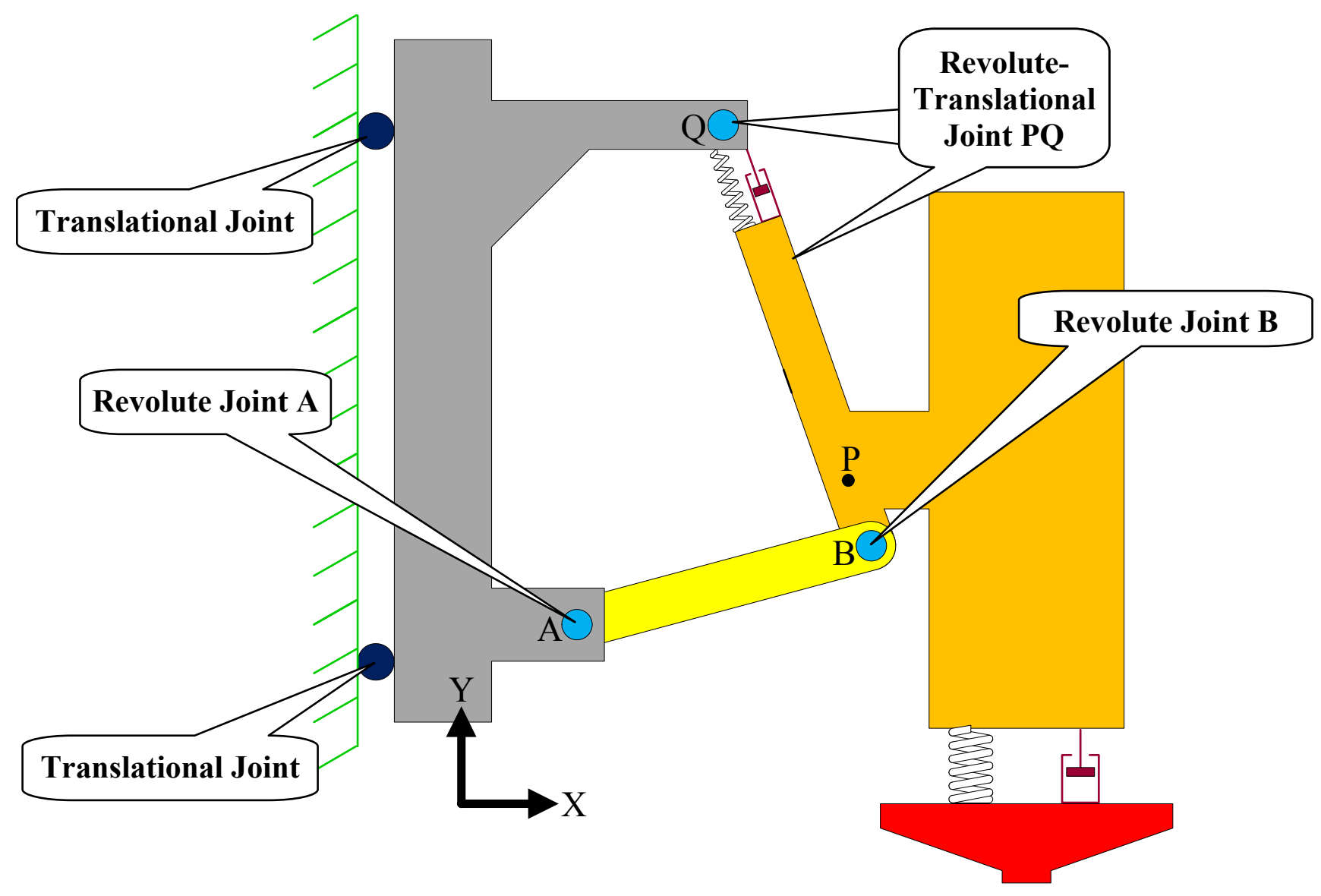

Figure 6-2 Non-Linear Model Joints

\subsection{Kinematic Assumptions}

Motions of the McPherson strut quarter-car suspension are assumed to be largely planar. For this reason the model is developed in $2 \mathrm{D}$ as opposed to $3 \mathrm{D}$. Because the model is developed in 2D and because the tie rod is lumped with the two-piece control arm, this model does not account for potential caster change or bump steer dynamics. Also, the links are assumed to be rigid and bushing deformation is neglected.

\subsection{Kinematic Constraints}

The following sections develop the kinematic constraints imposed by each joint. All constraint equations are developed relative to the global reference frame. The global X-Y 
coordinate origin is located at the intersection of $\mathrm{y}_{\mathrm{S}}^{\prime}$ and the static position of the horizontal edge of the hydraulic actuator, as seen in Figure 6-1.

\subsubsection{Translational Joint}

The sprung mass is constrained to motion in the vertical or global Y-direction by its translational joint. Accordingly, this joint prevents rotation and X-direction translation. The sprung mass orientation angle is constrained to zero. Since the sprung mass CG is a point on the global Y-axis, its global X-position is zero. The translational constraint vector representing this condition is:

$$
\boldsymbol{\Phi}^{\mathrm{t}}=\left[\begin{array}{c}
\phi_{\mathrm{S}} \\
x_{\mathrm{S}}
\end{array}\right]=0
$$

where $\phi_{\mathrm{S}}$ is sprung mass global orientation angle and $x_{\mathrm{S}}$ is the global X-position of the sprung mass. Figure 6-3 shows the sprung mass translational joint representation.

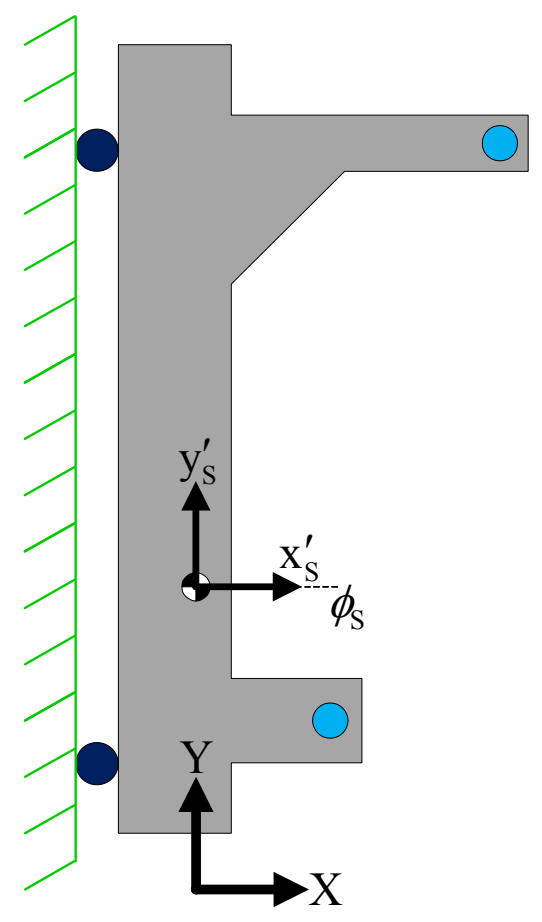

Figure 6-3 Translational Joint 


\subsubsection{Revolute Joint A}

The sprung mass is connected to the control arm by revolute joint A. This joint is represented by setting the vector that defines the position of the revolute joint via the sprung reference frame equal to the vector that defines the position of the revolute joint via the control arm reference frame. Figure 6-4 represents this vector addition graphically. The equation representing this vector addition is:

$$
\begin{aligned}
& \mathbf{r}_{\mathrm{S}}+\mathbf{A}_{\mathrm{S}} \mathbf{s}_{\mathrm{S}}^{\mathrm{A}}=\mathbf{r}_{\mathrm{C}}+\mathbf{A}_{\mathrm{C}} \mathbf{s}_{\mathrm{C}}^{\prime \mathrm{A}} \\
& {\left[\begin{array}{c}
x_{\mathrm{S}} \\
y_{\mathrm{S}}
\end{array}\right]+\left[\begin{array}{cc}
c \phi_{\mathrm{S}} & -s \phi_{\mathrm{S}} \\
s \phi_{\mathrm{S}} & c \phi_{\mathrm{S}}
\end{array}\right]\left[\begin{array}{c}
s_{\mathrm{Sx}}^{\prime \mathrm{A}} \\
s_{\mathrm{Sy}}^{\prime \mathrm{A}}
\end{array}\right]=\left[\begin{array}{c}
x_{\mathrm{C}} \\
y_{\mathrm{C}}
\end{array}\right]+\left[\begin{array}{cc}
c \phi_{\mathrm{C}} & -s \phi_{\mathrm{C}} \\
s \phi_{\mathrm{C}} & c \phi_{\mathrm{C}}
\end{array}\right]\left[\begin{array}{c}
s_{\mathrm{C} x}^{\prime \mathrm{A}} \\
s_{\mathrm{Cy}}^{\prime \mathrm{A}}
\end{array}\right]} \\
& x_{\mathrm{S}}+s_{\mathrm{S} x}^{\prime \mathrm{A}} c \phi_{\mathrm{S}}-s_{\mathrm{Sy}}^{\prime \mathrm{A}} s \phi_{\mathrm{S}}=x_{\mathrm{C}}+s_{\mathrm{C} x}^{\prime \mathrm{A}} c \phi_{\mathrm{C}}-s_{\mathrm{Cy}}^{\prime \mathrm{A}} s \phi_{\mathrm{C}} \\
& y_{\mathrm{S}}+s_{\mathrm{Sx}}^{\prime \mathrm{A}} s \phi_{\mathrm{S}}+s_{\mathrm{Sy}}^{\prime \mathrm{A}} c \phi_{\mathrm{S}}=y_{\mathrm{C}}+s_{\mathrm{C} x}^{\prime \mathrm{A}} s \phi_{\mathrm{C}}+s_{\mathrm{Cy}}^{\prime \mathrm{A}} c \phi_{\mathrm{C}}
\end{aligned}
$$

where $\mathbf{r}_{\mathrm{S}}$ is the vector from the global origin to the sprung mass CG, $\mathbf{A}_{\mathrm{S}}$ is the transformation matrix from the local $x_{\mathrm{S}}{ }^{\prime}-y_{\mathrm{S}}{ }^{\prime}$ sprung mass reference frame to the global reference frame, $\mathbf{s}_{\mathrm{S}}^{\mathrm{A}}$ is the local representation of the body fixed vector from the sprung mass CG to revolute joint $\mathrm{A}, \mathbf{r}_{\mathrm{C}}$ is the vector from the global origin to the control arm $\mathrm{CG}, \mathbf{A}_{\mathrm{C}}$ is the transformation matrix from the local $x_{\mathrm{C}}{ }^{\prime}-y_{\mathrm{C}}{ }^{\prime}$ control arm reference frame to the global reference frame, and $\mathbf{s}_{\mathrm{C}}^{\mathrm{A}}$ is the local representation of the body fixed vector from the control arm CG to revolute joint A. Likewise, $x_{\mathrm{S}}$ and $y_{\mathrm{S}}$ are the global components of $\mathbf{r}_{\mathrm{S}}, x_{\mathrm{C}}$ and $y_{\mathrm{C}}$ are the global components of $\mathbf{r}_{\mathrm{C}}, s_{\mathrm{Sx}}^{\prime \mathrm{A}}$ and $s_{\mathrm{Sy}}^{\prime \mathrm{A}}$ are the local $x_{\mathrm{S}}^{\prime}$ and $y_{\mathrm{S}}^{\prime}$ components of body fixed vector $\mathbf{s}_{\mathrm{S}}^{\prime \mathrm{A}}, s_{\mathrm{Cx}}^{\prime \mathrm{A}}$ and $s_{\mathrm{Cy}}^{\prime \mathrm{A}}$ are the local $x_{\mathrm{C}}{ }^{\prime}$ and $y_{\mathrm{C}}{ }^{\prime}$ components of body fixed vector $\mathbf{s}_{\mathrm{C}}^{\mathrm{A}}$, and $\phi_{\mathrm{S}}$ and $\phi_{\mathrm{C}}$ are the sprung mass and control arm global orientation angles.

Equation (6.4) can be written as the constraint vector representing revolute joint $\mathrm{A}$ as follows: 


$$
\boldsymbol{\Phi}^{\mathrm{RA}}=\left[\begin{array}{l}
x_{\mathrm{S}}+s_{\mathrm{Sx}}^{\prime \mathrm{A}} c \phi_{\mathrm{S}}-s_{\mathrm{Sy}}^{\prime \mathrm{A}} s \phi_{\mathrm{S}}-x_{\mathrm{C}}-s_{\mathrm{Cx}}^{\prime \mathrm{A}} c \phi_{\mathrm{C}}+s_{\mathrm{Cy}}^{\prime \mathrm{A}} s \phi_{\mathrm{C}} \\
y_{\mathrm{S}}+s_{\mathrm{Sx}}^{\prime \mathrm{A}} s \phi_{\mathrm{S}}+s_{\mathrm{Sy}}^{\prime \mathrm{A}} c \phi_{\mathrm{S}}-y_{\mathrm{C}}-s_{\mathrm{Cx}}^{\prime \mathrm{A}} s \phi_{\mathrm{C}}-s_{\mathrm{Cy}}^{\prime \mathrm{A}} c \phi_{\mathrm{C}}
\end{array}\right]=0
$$

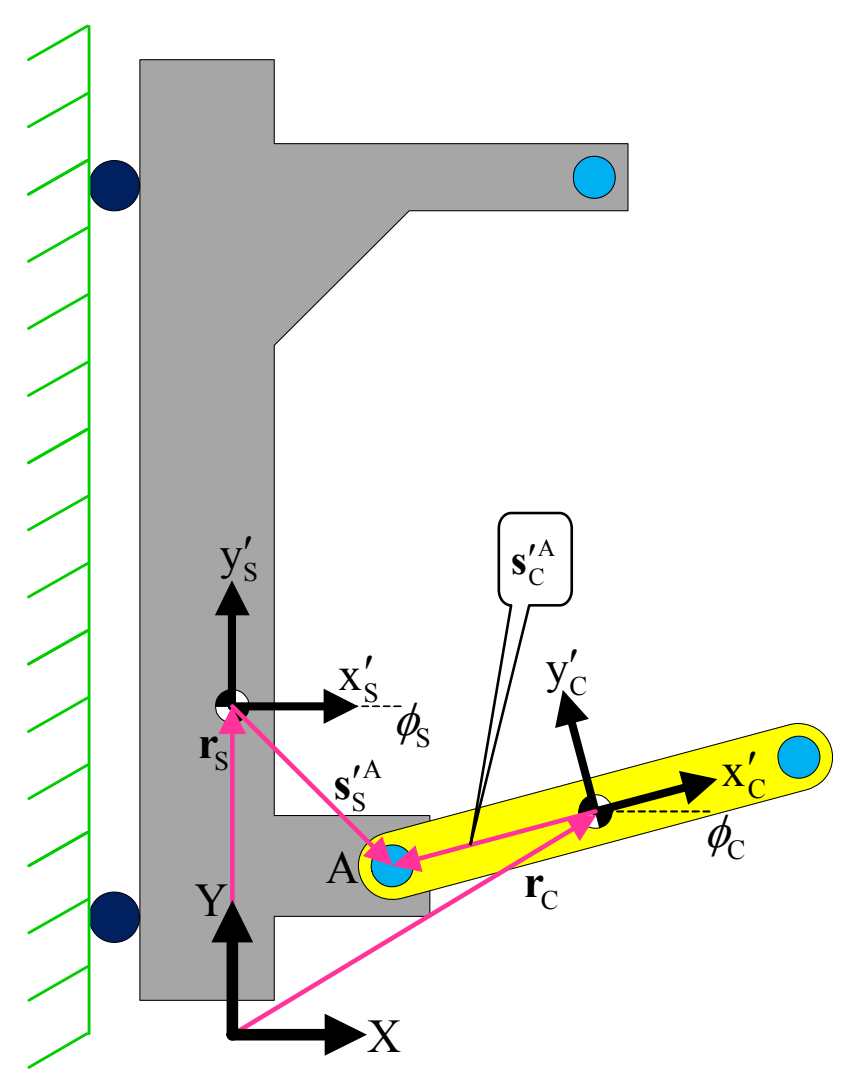

Figure 6-4 Revolute Joint A

\subsubsection{Revolute Joint B}

The unsprung mass is connected to the control arm by revolute joint B. This joint is represented by setting the vector that defines the position of the revolute joint via the control arm reference frame equal to the vector that defines the position of the revolute joint via the unsprung reference frame. Figure 6-5 represents this vector addition graphically. The equation representing this vector addition is: 


$$
\begin{aligned}
& \mathbf{r}_{\mathrm{C}}+\mathbf{A}_{\mathrm{C}} \mathbf{s}_{\mathrm{C}}^{\prime \mathrm{B}}=\mathbf{r}_{\mathrm{U}}+\mathbf{A}_{\mathrm{U}} \mathbf{s}_{\mathrm{U}}^{\mathrm{B}} \\
& {\left[\begin{array}{l}
x_{\mathrm{C}} \\
y_{\mathrm{C}}
\end{array}\right]+\left[\begin{array}{cc}
c \phi_{\mathrm{C}} & -s \phi_{\mathrm{C}} \\
s \phi_{\mathrm{C}} & c \phi_{\mathrm{C}}
\end{array}\right]\left[\begin{array}{l}
s_{\mathrm{C} x}^{\prime \mathrm{B}} \\
s_{\mathrm{C} y}^{\prime \mathrm{B}}
\end{array}\right]=\left[\begin{array}{c}
x_{\mathrm{U}} \\
y_{\mathrm{U}}
\end{array}\right]+\left[\begin{array}{cc}
c \phi_{\mathrm{U}} & -s \phi_{\mathrm{U}} \\
s \phi_{\mathrm{U}} & c \phi_{\mathrm{U}}
\end{array}\right]\left[\begin{array}{c}
s_{\mathrm{U} x}^{\prime \mathrm{B}} \\
s_{\mathrm{U} y}^{\prime \mathrm{B}}
\end{array}\right]} \\
& x_{\mathrm{C}}+s_{\mathrm{C} x}^{\prime \mathrm{B}} c \phi_{\mathrm{C}}-s_{\mathrm{C} y}^{\prime \mathrm{B}} s \phi_{\mathrm{C}}=x_{\mathrm{U}}+s_{\mathrm{U} x}^{\prime \mathrm{B}} c \phi_{\mathrm{U}}-s_{\mathrm{U} y}^{\prime \mathrm{B}} s \phi_{\mathrm{U}} \\
& y_{\mathrm{C}}+s_{\mathrm{C} x}^{\prime \mathrm{B}} s \phi_{\mathrm{C}}+s_{\mathrm{C} y}^{\prime \mathrm{B}} c \phi_{\mathrm{C}}=y_{\mathrm{U}}+s_{\mathrm{U} x}^{\prime \mathrm{B}} s \phi_{\mathrm{U}}+s_{\mathrm{U} y}^{\prime \mathrm{B}} c \phi_{\mathrm{U}}
\end{aligned}
$$

where $\mathbf{r}_{\mathrm{C}}, \mathbf{A}_{\mathrm{C}}, x_{\mathrm{C}}, y_{\mathrm{C}}$, and $\phi_{\mathrm{C}}$ are the same as defined for revolute joint A. $\mathbf{s}_{\mathrm{C}}^{\prime \mathrm{B}}$ is the local representation of the body fixed vector from the control arm CG to revolute joint $\mathrm{B}$, $\mathbf{r}_{U}$ is the vector from the global origin to the unsprung mass $C G, \mathbf{A}_{U}$ is the transformation matrix from the local $x_{U}{ }^{\prime}-y_{U}{ }^{\prime}$ unsprung mass reference frame to the global reference frame, and $\mathbf{s}_{\mathrm{U}}^{\mathrm{B}}$ is the local representation of the body fixed vector from the unsprung mass $\mathrm{CG}$ to revolute joint $\mathrm{B}$. Likewise, $s_{\mathrm{C} x}^{\prime \mathrm{B}}$ and $s_{\mathrm{Cy}}^{\prime \mathrm{B}}$ are the local $x_{\mathrm{C}}{ }^{\prime}$ and $y_{\mathrm{C}}{ }^{\prime}$ components of body fixed vector $\mathbf{s}_{\mathrm{C}}^{\prime \mathrm{B}}, x_{\mathrm{U}}$ and $y_{\mathrm{U}}$ are the global components of $\mathbf{r}_{\mathrm{U}}$, $\phi_{\mathrm{U}}$ is the unsprung mass global orientation angle, and ${s_{\mathrm{Ux}}^{\prime}}^{\mathrm{B}}$ and $s_{\mathrm{Uy}}^{\prime \mathrm{B}}$ are the local $x_{\mathrm{U}}{ }^{\prime}$ and $y_{\mathrm{U}}^{\prime}$ components of body fixed vector $\mathbf{s}_{\mathrm{U}}^{\prime \mathrm{B}}$. 


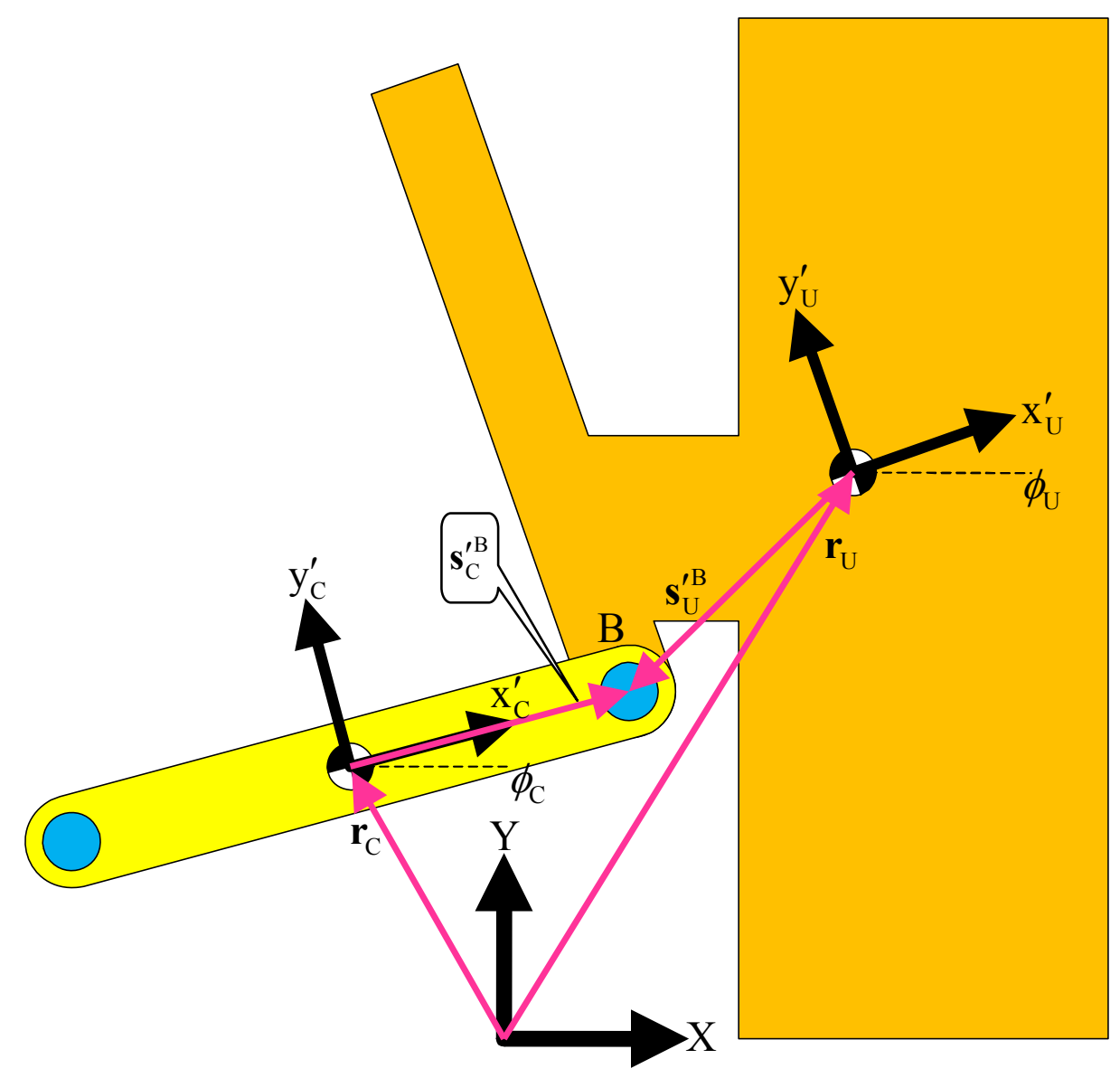

Figure 6-5 Revolute Joint B

Equation (6.6) can be written as the constraint vector representing revolute joint B as follows:

$$
\boldsymbol{\Phi}^{\mathrm{RB}}=\left[\begin{array}{l}
x_{\mathrm{C}}+s_{\mathrm{C} x}^{\prime \mathrm{B}} c \phi_{\mathrm{C}}-s_{\mathrm{Cy}}^{\prime \mathrm{B}} s \phi_{\mathrm{C}}-x_{\mathrm{U}}-s_{\mathrm{U} x}^{\prime \mathrm{B}} c \phi_{\mathrm{U}}+s_{\mathrm{Uy}}^{\prime \mathrm{B}} s \phi_{\mathrm{U}} \\
y_{\mathrm{C}}+s_{\mathrm{C} x}^{\prime \mathrm{B}} s \phi_{\mathrm{C}}+s_{\mathrm{Cy}}^{\prime \mathrm{B}} c \phi_{\mathrm{C}}-y_{\mathrm{U}}-s_{\mathrm{U} x}^{\prime \mathrm{B}} s \phi_{\mathrm{U}}-s_{\mathrm{U} y}^{\prime \mathrm{B}} c \phi_{\mathrm{U}}
\end{array}\right]=0
$$

\subsubsection{Revolute-Translational Joint PQ}

The sprung and unsprung masses are connected by revolute-translational composite joint PQ as seen in Figure 6-6. 


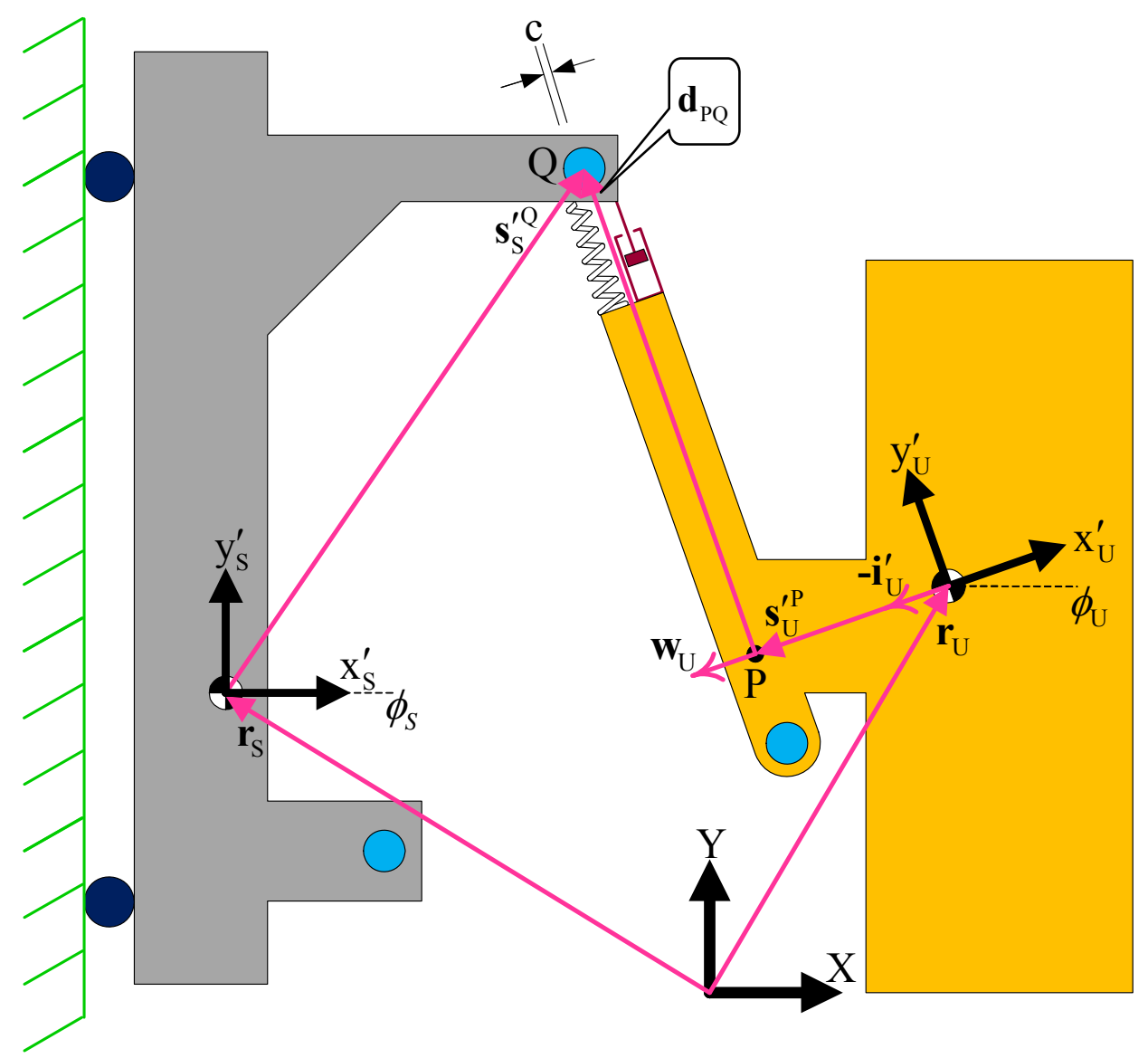

Figure 6-6 Revolute-Translational Joint

To formulate this joint condition, the scalar product of unit vector $\mathbf{w}_{U}$, a vector orthogonal to the strut's line of translation, with the vector $\mathbf{d}_{\mathrm{PQ}}$, a vector along the strut's line of translation, is set equal to the magnitude $\mathrm{C}$ of the projection of $\mathbf{d}_{\mathrm{PQ}}$ in the $\mathbf{w}_{\mathrm{U}}$ direction. Mathematically, this projection can be written as the following scalar product:

$$
\mathbf{w}_{\mathrm{U}}^{\mathrm{T}} \bullet \mathbf{d}_{\mathrm{PQ}}=C
$$

Unit vector $\mathbf{w}_{\mathrm{U}}$ is parallel to the negative $x_{\mathrm{U}}^{\prime}$-axis and thus equal to the negative of its base vector, $-\mathrm{i}_{\mathrm{U}}$. In global coordinates, $\mathbf{w}_{\mathrm{U}}$ is represented as: 


$$
\mathbf{w}_{\mathrm{U}}=-\mathbf{i}_{\mathrm{U}}=\mathbf{A}_{\mathrm{U}}\left(-\mathbf{i}_{\mathrm{U}}^{\prime}\right)=\left[\begin{array}{cc}
c \phi_{\mathrm{U}} & -s \phi_{\mathrm{U}} \\
s \phi_{\mathrm{U}} & c \phi_{\mathrm{U}}
\end{array}\right]\left[\begin{array}{c}
-1 \\
0
\end{array}\right]=\left[\begin{array}{c}
-c \phi_{\mathrm{U}} \\
-s \phi_{\mathrm{U}}
\end{array}\right]
$$

By vector addition, the vector $\mathbf{d}_{\mathrm{PQ}}$ from point $\mathrm{P}$ to revolute joint $\mathrm{Q}$ is:

$$
\begin{aligned}
\mathbf{d}_{\mathrm{PQ}} & =\mathbf{r}_{\mathrm{S}}+\mathbf{A}_{\mathrm{S}} \mathbf{s}_{\mathrm{S}}^{\prime \mathrm{Q}}-\mathbf{r}_{\mathrm{U}}-\mathbf{A}_{\mathrm{U}} \mathbf{s}_{\mathrm{U}}^{\prime \mathrm{P}} \\
\mathbf{d}_{\mathrm{PQ}} & =\left[\begin{array}{l}
x_{\mathrm{S}} \\
y_{\mathrm{S}}
\end{array}\right]+\left[\begin{array}{cc}
c \phi_{\mathrm{S}} & -s \phi_{\mathrm{S}} \\
s \phi_{\mathrm{S}} & c \phi_{\mathrm{S}}
\end{array}\right]\left[\begin{array}{c}
s_{\mathrm{Sx}}^{\prime \mathrm{Q}} \\
s_{\mathrm{Sy}}^{\prime \mathrm{Q}}
\end{array}\right]-\left[\begin{array}{c}
x_{\mathrm{U}} \\
y_{\mathrm{U}}
\end{array}\right]-\left[\begin{array}{cc}
c \phi_{\mathrm{U}} & -s \phi_{\mathrm{U}} \\
s \phi_{\mathrm{U}} & c \phi_{\mathrm{U}}
\end{array}\right]\left[\begin{array}{c}
s_{\mathrm{Ux}}^{\prime \mathrm{P}} \\
s_{\mathrm{Uy}}^{\prime \mathrm{P}}
\end{array}\right] \\
\mathbf{d}_{\mathrm{PQ}}= & {\left[\begin{array}{l}
x_{\mathrm{S}}+s_{\mathrm{Sx}}^{\prime \mathrm{Q}} c \phi_{\mathrm{S}}-s_{\mathrm{Sy}}^{\prime \mathrm{Q}} s \phi_{\mathrm{S}}-x_{\mathrm{U}}-s_{\mathrm{Ux}}^{\prime \mathrm{P}} c \phi_{\mathrm{U}}+s_{\mathrm{Uy}}^{\prime \mathrm{P}} s \phi_{\mathrm{U}} \\
y_{\mathrm{S}}+s_{\mathrm{Sx}}^{\prime \mathrm{Q}} s \phi_{\mathrm{S}}+s_{\mathrm{Sy}}^{\prime \mathrm{Q}} c \phi_{\mathrm{S}}-y_{\mathrm{U}}-s_{\mathrm{Ux}}^{\prime \mathrm{P}} s \phi_{\mathrm{U}}-s_{\mathrm{Uy}}^{\prime \mathrm{P}} c \phi_{\mathrm{U}}
\end{array}\right] }
\end{aligned}
$$

where $\mathbf{r}_{\mathrm{S}}, x_{\mathrm{S}}, y_{\mathrm{S}}, \mathbf{A}_{\mathrm{S}}, \phi_{\mathrm{S}}, \mathbf{r}_{\mathrm{U}}, x_{\mathrm{U}}, y_{\mathrm{U}}, \mathbf{A}_{\mathrm{U}}$, and $\phi_{\mathrm{U}}$ are the same as defined for previous joints. $\mathbf{s}_{\mathrm{S}}^{\mathrm{Q}}$ is the local representation of the body fixed vector from the sprung mass $\mathrm{CG}$ to revolute joint $\mathrm{Q}$ and $\mathbf{s}_{\mathrm{U}}^{\mathrm{P}}$ is the local representation of the body fixed vector from the unsprung mass CG to point $\mathrm{P}$ on strut's translation line. Likewise, $s_{\mathrm{Sx}}^{\prime \mathrm{Q}}$ and $s_{\mathrm{Sy}}^{\prime \mathrm{Q}}$ are the local $x_{\mathrm{S}}^{\prime}$ and $y_{\mathrm{S}}^{\prime}$ components of body fixed vector $\mathbf{s}_{\mathrm{S}}^{\prime \mathrm{Q}}$ and $s_{\mathrm{Ux}}^{\prime \mathrm{P}}$ and $s_{\mathrm{Uy}}^{\prime \mathrm{P}}$ are the local $x_{\mathrm{U}}^{\prime}$ and $y_{\mathrm{U}}^{\prime}$ components of body fixed vector $\mathbf{s}_{\mathrm{U}}^{\mathrm{P}}$.

Noting that $s_{\mathrm{Ux}}^{\prime \mathrm{P}}=s_{\mathrm{Ux}}^{\prime \mathrm{B}}$, the vector $\mathbf{d}_{\mathrm{PQ}}$ can be written as follows:

$$
\mathbf{d}_{\mathrm{PQ}}=\left[\begin{array}{l}
x_{\mathrm{S}}+s_{\mathrm{Sx}}^{\prime \mathrm{Q}} c \phi_{\mathrm{S}}-s_{\mathrm{Sy}}^{\prime \mathrm{Q}} s \phi_{\mathrm{S}}-x_{\mathrm{U}}-s_{\mathrm{Ux}}^{\prime \mathrm{B}} c \phi_{\mathrm{U}}+s_{\mathrm{Uy}}^{\prime \mathrm{P}} s \phi_{\mathrm{U}} \\
y_{\mathrm{S}}+s_{\mathrm{Sx}}^{\prime \mathrm{Q}} s \phi_{\mathrm{S}}+s_{\mathrm{Sy}}^{\prime \mathrm{Q}} c \phi_{\mathrm{S}}-y_{\mathrm{U}}-s_{\mathrm{Ux}}^{\prime \mathrm{B}} s \phi_{\mathrm{U}}-s_{\mathrm{Uy}}^{\prime \mathrm{P}} c \phi_{\mathrm{U}}
\end{array}\right]
$$

Since $\mathbf{d}_{\mathrm{PQ}}$ is directed along the line of translation, it has no component in the $\mathbf{w}_{\mathrm{U}}$ direction and the magnitude of its projection on $\mathbf{w}_{\mathrm{U}}$ is zero $(\mathrm{C}=0)$. Thus the constraint representing the revolute-translational composite joint can be written as follows: 


$$
\begin{aligned}
& \Phi^{\mathrm{RT}}= \mathbf{w}_{\mathrm{U}}^{\mathrm{T}} \bullet \mathbf{d}_{\mathrm{PQ}}-C=0 \\
& \Phi^{\mathrm{RT}}=\left[\begin{array}{ll}
-c \phi_{\mathrm{U}} & -s \phi_{\mathrm{U}}
\end{array}\right] \bullet\left[\begin{array}{l}
x_{\mathrm{S}}+s_{\mathrm{Sx}}^{\prime \mathrm{Q}} c \phi_{\mathrm{S}}-s_{\mathrm{Sy}}^{\prime \mathrm{Q}} s \phi_{\mathrm{S}}-x_{\mathrm{U}}-s_{\mathrm{Ux}}^{\prime \mathrm{B}} c \phi_{\mathrm{U}}+s_{\mathrm{Uy}}^{\prime \mathrm{P}} s \phi_{\mathrm{U}} \\
y_{\mathrm{S}}+s_{\mathrm{Sx}}^{\prime \mathrm{Q}} s \phi_{\mathrm{S}}+s_{\mathrm{Sy}}^{\prime \mathrm{Q}} c \phi_{\mathrm{S}}-y_{\mathrm{U}}-s_{\mathrm{U} x}^{\prime \mathrm{B}} s \phi_{\mathrm{U}}-s_{\mathrm{Uy}}^{\prime \mathrm{P}} c \phi_{\mathrm{U}}
\end{array}\right]-0=0 \\
& \Phi^{\mathrm{RT}}=-c \phi_{\mathrm{U}}\left(x_{\mathrm{S}}+s_{\mathrm{Sx}}^{\prime \mathrm{Q}} c \phi_{\mathrm{S}}-s_{\mathrm{Sy}}^{\prime \mathrm{Q}} s \phi_{\mathrm{S}}-x_{\mathrm{U}}-{s_{\mathrm{U}}^{\prime}}^{\mathrm{B}} c \phi_{\mathrm{U}}+s_{\mathrm{Uy}}^{\prime \mathrm{P}} s \phi_{\mathrm{U}}\right)-\ldots \\
& s \phi_{\mathrm{U}}\left(y_{\mathrm{S}}+s_{\mathrm{Sx}}^{\prime \mathrm{Q}} s \phi_{\mathrm{S}}+s_{\mathrm{Sy}}^{\prime \mathrm{Q}} c \phi_{\mathrm{S}}-y_{\mathrm{U}}-s_{\mathrm{U} x}^{\prime \mathrm{B}} s \phi_{\mathrm{U}}-s_{\mathrm{Uy}}^{\prime \mathrm{P}} c \phi_{\mathrm{U}}\right)=0
\end{aligned}
$$

\subsection{Driving Constraint}

The actuator coordinate $y_{\mathrm{A}}$ is the driven DOF. In general, this driven DOF could be a function of time and the model's coordinates: $y_{\mathrm{A}}=f\left(t, x_{\mathrm{S}}, y_{\mathrm{S}}, \phi_{\mathrm{S}}, x_{\mathrm{C}}, y_{\mathrm{C}}, \phi_{\mathrm{C}}, x_{\mathrm{U}}, y_{\mathrm{U}}, \phi_{\mathrm{U}}\right)$. For this model the actuator position is a function only of time: $y_{\mathrm{A}}=f(t)$, thus the driving constraint is:

$$
\Phi^{\mathrm{D}}=\left[y_{\mathrm{A}}-f(t)\right]=0
$$

\subsection{Complete Constraint Vector}

The complete constraint vector is a combination of the kinematic and driving constraints:

$$
\boldsymbol{\Phi}=\left[\begin{array}{c}
\boldsymbol{\Phi}^{\mathrm{K}} \\
\boldsymbol{\Phi}^{\mathrm{D}}
\end{array}\right]=\left[\begin{array}{c}
\boldsymbol{\Phi}^{\mathrm{t}} \\
\boldsymbol{\Phi}^{\mathrm{RA}} \\
\boldsymbol{\Phi}^{\mathrm{RB}} \\
\Phi^{\mathrm{RT}} \\
\Phi^{\mathrm{D}}
\end{array}\right]=\left[\begin{array}{c}
\phi_{\mathrm{S}} \\
x_{\mathrm{S}} \\
x_{\mathrm{S}}+s_{\mathrm{Sx}}^{\prime \mathrm{A}} c \phi_{\mathrm{S}}-s_{\mathrm{Sy}}^{\prime \mathrm{A}} s \phi_{\mathrm{S}}-x_{\mathrm{C}}-s_{\mathrm{Cx}}^{\prime \mathrm{A}} c \phi_{\mathrm{C}}+s_{\mathrm{Cy}}^{\prime \mathrm{A}} s \phi_{\mathrm{C}} \\
y_{\mathrm{S}}+s_{\mathrm{Sx}}^{\prime \mathrm{A}} s \phi_{\mathrm{S}}+s_{\mathrm{Sy}}^{\prime \mathrm{A}} c \phi_{\mathrm{S}}-y_{\mathrm{C}}-s_{\mathrm{Cx}}^{\prime \mathrm{A}} s \phi_{\mathrm{C}}-s_{\mathrm{Cy}}^{\prime \mathrm{A}} c \phi_{\mathrm{C}} \\
x_{\mathrm{C}}+s_{\mathrm{Cx}}^{\prime \mathrm{B}} c \phi_{\mathrm{C}}-s_{\mathrm{Cy}}^{\prime \mathrm{B}} s \phi_{\mathrm{C}}-x_{\mathrm{U}}-s_{\mathrm{Ux}}^{\prime \mathrm{B}} c \phi_{\mathrm{U}}+s_{\mathrm{Uy}}^{\prime \mathrm{B}} s \phi_{\mathrm{U}} \\
y_{\mathrm{C}}+s_{\mathrm{C} x}^{\prime \mathrm{B}} s \phi_{\mathrm{C}}+s_{\mathrm{Cy}}^{\prime \mathrm{B}} c \phi_{\mathrm{C}}-y_{\mathrm{U}}-s_{\mathrm{Ux}}^{\prime \mathrm{B}} s \phi_{\mathrm{U}}-s_{\mathrm{Uy}}^{\prime \mathrm{B}} c \phi_{\mathrm{U}} \\
-c \phi_{\mathrm{U}}\left(x_{\mathrm{S}}+s_{\mathrm{Sx}}^{\prime \mathrm{Q}} c \phi_{\mathrm{S}}-s_{\mathrm{Sy}}^{\prime \mathrm{Q}} s \phi_{\mathrm{S}}-x_{\mathrm{U}}-s_{\mathrm{Ux}}^{\prime \mathrm{B}} c \phi_{\mathrm{U}}+s_{\mathrm{Uy}}^{\prime \mathrm{P}} s \phi_{\mathrm{U}}\right)-\ldots \\
s \phi_{\mathrm{U}}\left(y_{\mathrm{S}}+s_{\mathrm{Sx}}^{\prime \mathrm{Q}} s \phi_{\mathrm{S}}+s_{\mathrm{Sy}}^{\prime \mathrm{Q}} c \phi_{\mathrm{S}}-y_{\mathrm{U}}-s_{\mathrm{Ux}}^{\prime \mathrm{B}} s \phi_{\mathrm{U}}-s_{\mathrm{Uy}}^{\prime \mathrm{P}} c \phi_{\mathrm{U}}\right) \\
y_{\mathrm{A}}-f(t)
\end{array}\right\}=0
$$


For notational compactness the constants are re-named and substituted into $\boldsymbol{\Phi}$ as follows:

$$
\begin{aligned}
& \mathrm{d}=s_{\mathrm{Sx}}^{\prime \mathrm{A}} \\
& \mathrm{e}=s_{\mathrm{S}}^{\prime \mathrm{A}} \\
& \mathrm{f}=s_{\mathrm{Cx}}^{\prime \mathrm{A}} \\
& \mathrm{g}=s_{\mathrm{Cy}}^{\prime \mathrm{A}} \\
& \mathrm{h}=s_{\mathrm{Cx}}^{\prime \mathrm{B}} \\
& \mathrm{i}=\mathrm{s}_{\mathrm{Cy}}^{\prime \mathrm{B}} \\
& \mathrm{j}=\mathrm{s}_{\mathrm{Ux}}^{\prime \mathrm{B}} \\
& \mathrm{k}=\mathrm{s}_{\mathrm{Uy}}^{\prime \mathrm{B}} \\
& \mathrm{l}=s_{\mathrm{Sx}}^{\prime \mathrm{Q}} \\
& \mathrm{m}=s_{\mathrm{Sy}}^{\prime \mathrm{Q}} \\
& \mathrm{n}=s_{\mathrm{Uy}}^{\prime \mathrm{P}}
\end{aligned} \quad\left[\begin{array}{c}
\phi_{\mathrm{S}} \\
x_{\mathrm{S}}+\mathrm{d} c \phi_{\mathrm{S}}-\mathrm{es} \phi_{\mathrm{S}}-x_{\mathrm{C}}-\mathrm{f} c \phi_{\mathrm{C}}+\mathrm{g} s \phi_{\mathrm{C}} \\
y_{\mathrm{S}}+\mathrm{d} s \phi_{\mathrm{S}}+\mathrm{e} c \phi_{\mathrm{S}}-y_{\mathrm{C}}-\mathrm{fs} \phi_{\mathrm{C}}-\mathrm{g} c \phi_{\mathrm{C}} \\
x_{\mathrm{C}}+\mathrm{h} c \phi_{\mathrm{C}}-\mathrm{i} s \phi_{\mathrm{C}}-x_{\mathrm{U}}-\mathrm{j} c \phi_{\mathrm{U}}+\mathrm{ks} \phi_{\mathrm{U}} \\
y_{\mathrm{C}}+\mathrm{h} s \phi_{\mathrm{C}}+\mathrm{i} c \phi_{\mathrm{C}}-y_{\mathrm{U}}-\mathrm{j} s \phi_{\mathrm{U}}-\mathrm{k} c \phi_{\mathrm{U}} \\
-c \phi_{\mathrm{U}}\left(x_{\mathrm{S}}+\mathrm{l} c \phi_{\mathrm{S}}-\mathrm{ms} \phi_{\mathrm{S}}-x_{\mathrm{U}}-\mathrm{j} c \phi_{\mathrm{U}}+\mathrm{n} s \phi_{\mathrm{U}}\right)-\ldots \\
s \phi_{\mathrm{U}}\left(y_{\mathrm{S}}+\mathrm{l} s \phi_{\mathrm{S}}+\mathrm{m} c \phi_{\mathrm{S}}-y_{\mathrm{U}}-\mathrm{j} s \phi_{\mathrm{U}}-\mathrm{n} c \phi_{\mathrm{U}}\right) \\
y_{\mathrm{A}}-f(t)
\end{array}\right]=0
$$

The Jacobian of this constraint vector is:

$$
\begin{aligned}
\boldsymbol{\Phi}_{\mathrm{q}} & =\left[\begin{array}{cccccccccc}
0 & 0 & 1 & 0 & 0 & 0 & 0 & 0 & 0 & 0 \\
1 & 0 & 0 & 0 & 0 & 0 & 0 & 0 & 0 & 0 \\
1 & 0 & -\mathrm{d} s \phi_{\mathrm{S}}-\mathrm{e} c \phi_{\mathrm{S}} & -1 & 0 & \mathrm{fs} \phi_{\mathrm{C}}+\mathrm{g} c \phi_{\mathrm{C}} & 0 & 0 & 0 & 0 \\
0 & 1 & \mathrm{~d} c \phi_{\mathrm{S}}-\mathrm{es} \phi_{\mathrm{S}} & 0 & -1 & -\mathrm{f} c \phi_{\mathrm{C}}+\mathrm{g} s \phi_{\mathrm{C}} & 0 & 0 & 0 & 0 \\
0 & 0 & 0 & 1 & 0 & -\mathrm{h} s \phi_{\mathrm{C}}-\mathrm{i} c \phi_{\mathrm{C}} & -1 & 0 & \mathrm{j} s \phi_{\mathrm{U}}+\mathrm{k} c \phi_{\mathrm{U}} & 0 \\
0 & 0 & 0 & 0 & 1 & \mathrm{~h} c \phi_{\mathrm{C}}-\mathrm{i} s \phi_{\mathrm{C}} & 0 & -1 & -\mathrm{j} c \phi_{\mathrm{U}}+\mathrm{k} s \phi_{\mathrm{U}} & 0 \\
-c \phi_{\mathrm{U}} & -\mathrm{s} \phi_{\mathrm{U}} & \mathrm{R}_{1} & 0 & 0 & 0 & c \phi_{\mathrm{U}} & s \phi_{\mathrm{U}} & \mathrm{R}_{2} & 0 \\
0 & 0 & 0 & 0 & 0 & 0 & 0 & 0 & 0 & 1
\end{array}\right] \\
\mathrm{R}_{1} & =1 s\left(-\phi_{\mathrm{U}}+\phi_{\mathrm{S}}\right)+\mathrm{m} c\left(-\phi_{\mathrm{U}}+\phi_{\mathrm{S}}\right) \\
\mathrm{R}_{2} & =\mathrm{x}_{\mathrm{s}} s \phi_{\mathrm{U}}-1 \mathrm{l}\left(-\phi_{\mathrm{U}}+\phi_{\mathrm{S}}\right)-\mathrm{m} c\left(-\phi_{\mathrm{U}}+\phi_{\mathrm{S}}\right)-\mathrm{x}_{\mathrm{u}} s \phi_{\mathrm{U}}-\mathrm{y}_{\mathrm{s}} c \phi_{\mathrm{U}}+\mathrm{y}_{\mathrm{u}} c \phi_{\mathrm{U}}
\end{aligned}
$$

This Jacobian has full-row rank, and therefore the constraint equations are independent; that is, the system is not over-constrained. 


\subsection{Acceleration Equations}

The acceleration equation can be obtained [7] as:

$$
\boldsymbol{\alpha}=\Phi_{\mathrm{q}} \ddot{\mathbf{q}}=-\left(\boldsymbol{\Phi}_{\mathrm{q}} \dot{\mathbf{q}}\right)_{\mathrm{q}} \dot{\mathbf{q}}-\mathbf{2} \Phi_{\mathrm{qt}} \dot{\mathbf{q}}-\Phi_{\mathrm{tt}}
$$

It is foreseeable that a variety of driving inputs will be used to drive the quarter-car's actuator. Therefore the function $f(t)$ defining this input is kept general. Adhering to this idea, the $\boldsymbol{\Phi}_{\mathrm{tt}}$ term is:

$$
\boldsymbol{\Phi}_{\mathrm{tt}}=\left[\begin{array}{c}
0 \\
0 \\
0 \\
0 \\
0 \\
0 \\
0 \\
\frac{d^{2} f(t)}{d t^{2}}
\end{array}\right]
$$

Because $\boldsymbol{\Phi}_{\mathrm{q}}$ has no explicitly time dependent terms, $\boldsymbol{\Phi}_{\mathrm{qt}}$ is zero. The last acceleration term, $\left(\boldsymbol{\Phi}_{\mathrm{q}} \dot{\mathbf{q}}\right)_{\mathrm{q}} \dot{\mathbf{q}}$, is solved below: 


$$
\begin{aligned}
& \left(\boldsymbol{\Phi}_{\mathrm{q}} \dot{\mathbf{q}}\right)_{\mathrm{q}} \dot{\mathbf{q}}=\left[\begin{array}{c}
0 \\
0 \\
\mathrm{R}_{3} \\
\mathrm{R}_{4} \\
\mathrm{R}_{5} \\
\mathrm{R}_{6} \\
\mathrm{R}_{7} \\
0
\end{array}\right] \\
& \mathrm{R}_{3}=-\dot{\phi}_{\mathrm{S}}^{2} \mathrm{~d} c \phi_{\mathrm{S}}+\dot{\phi}_{\mathrm{S}}^{2} \mathrm{es} \phi_{\mathrm{S}}+\dot{\phi}_{\mathrm{C}}^{2} \mathrm{f} c \phi_{\mathrm{C}}-\dot{\phi}_{\mathrm{C}}^{2} \mathrm{gs} \phi_{\mathrm{C}} \\
& \mathrm{R}_{4}=-\dot{\phi}_{\mathrm{S}}^{2} \mathrm{~d} s \phi_{\mathrm{S}}-\dot{\phi}_{\mathrm{S}}^{2} \mathrm{e} c \phi_{\mathrm{S}}+\dot{\phi}_{\mathrm{C}}^{2} \mathrm{f} s \phi_{\mathrm{C}}+\dot{\phi}_{\mathrm{C}}^{2} \mathrm{~g} c \phi_{\mathrm{C}} \\
& \mathrm{R}_{5}=-\dot{\phi}_{\mathrm{C}}^{2} \mathrm{~h} c \phi_{\mathrm{C}}+\dot{\phi}_{\mathrm{C}}^{2} \mathrm{i} s \phi_{\mathrm{C}}+\dot{\phi}_{\mathrm{U}}^{2} \mathrm{j} c \phi_{\mathrm{U}}-\dot{\phi}_{\mathrm{U}}^{2} \mathrm{k} s \phi_{\mathrm{U}} \\
& \mathrm{R}_{6}=-\dot{\phi}_{\mathrm{C}}^{2} \mathrm{~h} s \phi_{\mathrm{C}}-\dot{\phi}_{\mathrm{C}}^{2} \mathrm{i} c \phi_{\mathrm{C}}+\dot{\phi}_{\mathrm{U}}^{2} \mathrm{j} s \phi_{\mathrm{U}}+\dot{\phi}_{\mathrm{U}}^{2} \mathrm{k} c \phi_{\mathrm{U}} \\
& \mathrm{R}_{7}=2 \phi_{\mathrm{U}} s \phi_{\mathrm{U}} \dot{\mathrm{x}}_{\mathrm{s}}-2 \phi_{\mathrm{U}} c \phi_{\mathrm{U}} \dot{\mathrm{y}}_{\mathrm{s}}+\dot{\phi}_{\mathrm{S}}^{2} 1 c\left(-\phi_{\mathrm{U}}+\phi_{\mathrm{S}}\right)-\dot{\phi}_{\mathrm{S}}^{2} \mathrm{~ms}\left(-\phi_{\mathrm{U}}+\phi_{\mathrm{S}}\right)-\ldots \\
& 2 \dot{\phi}_{\mathrm{S}} \dot{\phi}_{\mathrm{U}} 1 c\left(-\phi_{\mathrm{U}}+\phi_{\mathrm{S}}\right)+2 \dot{\phi}_{\mathrm{S}} \dot{\phi}_{\mathrm{U}} \mathrm{ms}\left(-\phi_{\mathrm{U}}+\phi_{\mathrm{S}}\right)-2 \dot{\phi}_{\mathrm{U}} s \phi_{\mathrm{U}} \dot{\mathrm{x}}_{\mathrm{u}}+\ldots \\
& 2 \dot{\phi}_{\mathrm{U}} c \phi_{\mathrm{U}} \dot{\mathrm{y}}_{\mathrm{u}}+\dot{\phi}_{\mathrm{U}}^{2} l c\left(-\phi_{\mathrm{U}}+\phi_{\mathrm{S}}\right)-\dot{\phi}_{\mathrm{U}}^{2} \mathrm{~ms}\left(-\phi_{\mathrm{U}}+\phi_{\mathrm{S}}\right)+\ldots \\
& \dot{\phi}_{\mathrm{U}}^{2} s \phi_{\mathrm{U}} \mathrm{y}_{\mathrm{s}}-\dot{\phi}_{\mathrm{U}}^{2} s \phi_{\mathrm{U}} \mathrm{y}_{\mathrm{u}}+\dot{\phi}_{\mathrm{U}}^{2} c \phi_{\mathrm{U}} \mathrm{x}_{\mathrm{s}}-\dot{\phi}_{\mathrm{U}}^{2} c \phi_{\mathrm{U}} \mathrm{x}_{\mathrm{u}}
\end{aligned}
$$

Together, these terms form the acceleration equation:

$$
\boldsymbol{\alpha}=-\left(\boldsymbol{\Phi}_{\mathrm{q}} \dot{\mathbf{q}}\right)_{\mathrm{q}} \dot{\mathbf{q}}-\boldsymbol{\Phi}_{\mathrm{tt}}=-\left[\begin{array}{c}
0 \\
0 \\
\mathrm{R}_{3} \\
\mathrm{R}_{4} \\
\mathrm{R}_{5} \\
\mathrm{R}_{6} \\
\mathrm{R}_{7} \\
0
\end{array}\right]-\left[\begin{array}{c}
0 \\
0 \\
0 \\
0 \\
0 \\
0 \\
\frac{d^{2} f(t)}{d t^{2}}
\end{array}\right]=\left[\begin{array}{c}
0 \\
0 \\
-\mathrm{R}_{3} \\
-\mathrm{R}_{4} \\
-\mathrm{R}_{5} \\
-\mathrm{R}_{6} \\
-\mathrm{R}_{7} \\
-\frac{d^{2} f(t)}{d t^{2}}
\end{array}\right]
$$

\subsection{Applied Force Vector}

This section presents the calculation of the applied force vector, $\mathbf{Q}^{\mathrm{A}}$. Figure 6-7 shows the McPherson strut model used for this purpose. It shows the primary spring and 
damper connected to the sprung and unsprung bodies and the tire spring and damper connected to the unsprung and actuator bodies. These spring and damper force elements form this model's translational-spring-dampers (TSDs).

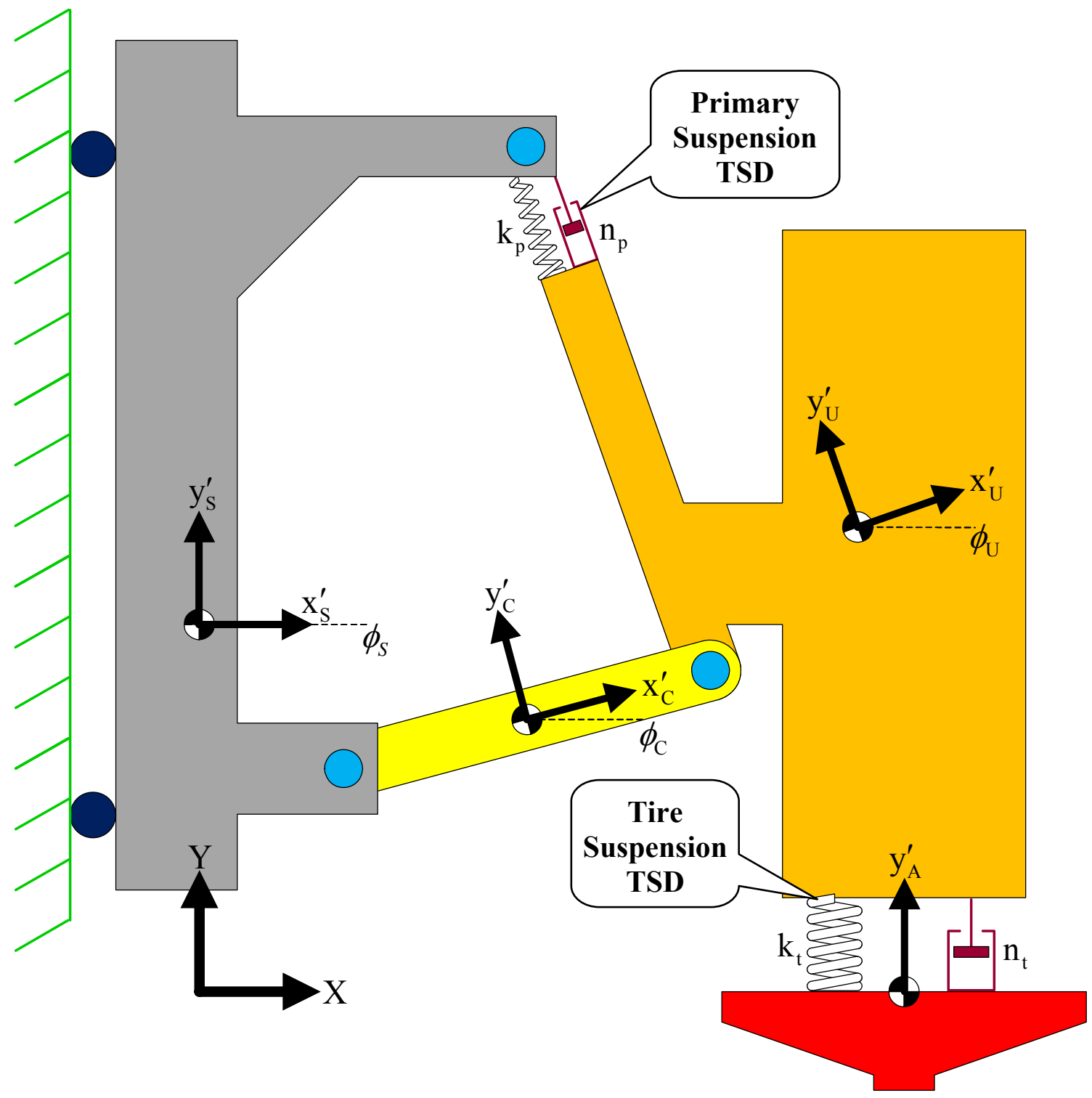

Figure 6-7 Non-Linear Model TSDs

The primary TSD approximates the McPherson strut's primary suspension stiffness and damping with a linear spring of stiffness $k_{p}$ and damper of damping value $n_{p}$. The tire 
TSD approximates the tire's stiffness and damping with a linear spring of stiffness $k_{t}$ and damper of damping value $n_{t}$.

The following sections symbolically solve for the non-linear model's force vector, $\mathbf{Q}^{\mathbf{A}}$. To begin with, forces corresponding to the primary and tire TSDs are developed. Next, individual force vectors are assembled from the sums of the forces and torques acting on each body. Lastly, the complete force vector $\mathbf{Q}^{\mathrm{A}}$ is populated from each body's individual force vector. 


\subsubsection{Primary TSD}

This section develops the force created by the primary TSD. The primary TSD, shown in Figure 6-8, produces a force $\mathrm{F}^{\mathrm{P}}$ along the vector $\mathbf{d}_{\mathrm{PQ}}$ in proportion to its compression $\Delta \mathrm{d}_{\mathrm{PQ}}$ and compression velocity $\Delta \dot{\mathrm{d}}_{\mathrm{PQ}}$.

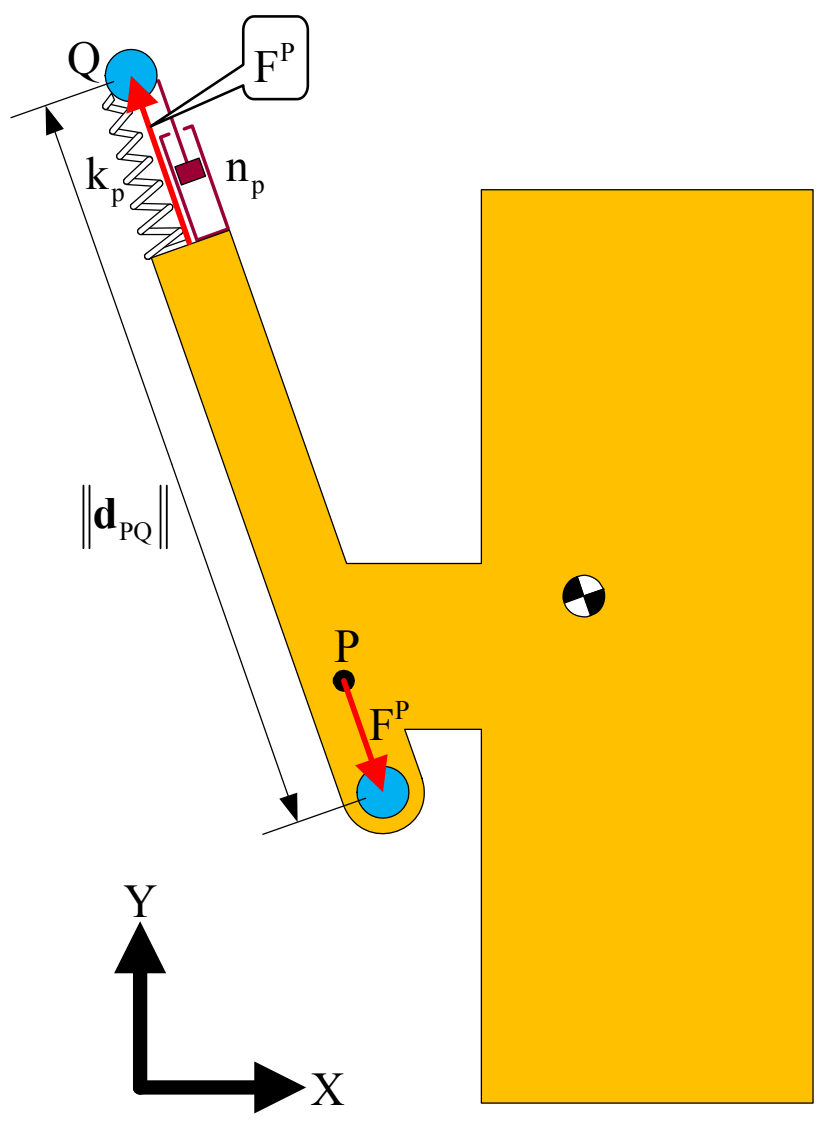

Figure 6-8 Non-Linear Model Primary TSD

To specify the primary TSD compression, its initial and displaced length must be specified. The initial length, $\mathrm{d}_{\mathrm{PQ}_{\mathrm{I}}}$, is its length without any load on it, or free length. The displaced length, $\mathrm{d}_{\mathrm{PQ}_{\mathrm{D}}}$, is the current length or magnitude of vector $\mathbf{d}_{\mathrm{PQ}}$. 


$$
\begin{aligned}
& \text { Displaced length }=\mathrm{d}_{\mathrm{PQ}_{\mathrm{D}}}=\left\|\mathbf{d}_{\mathrm{PQ}}\right\|= \\
& \mathrm{d}_{\mathrm{PQ}_{\mathrm{D}}}=\left\|\begin{array}{l}
x_{\mathrm{S}}+s_{\mathrm{Sx}}^{\prime \mathrm{Q}} c \phi_{\mathrm{S}}-s_{\mathrm{Sy}}^{\prime \mathrm{Q}} s \phi_{\mathrm{S}}-x_{\mathrm{U}}-{s_{\mathrm{Ux}}^{\prime \mathrm{B}}} c \phi_{\mathrm{U}}+s_{\mathrm{Uy}}^{\prime \mathrm{P}} s \phi_{\mathrm{U}} \\
y_{\mathrm{S}}+s_{\mathrm{Sx}}^{\prime \mathrm{Q}} s \phi_{\mathrm{S}}+s_{\mathrm{Sy}}^{\prime \mathrm{Q}} c \phi_{\mathrm{S}}-y_{\mathrm{U}}-s_{\mathrm{Ux}}^{\prime \mathrm{B}} s \phi_{\mathrm{U}}-s_{\mathrm{Uy}}^{\prime \mathrm{P}} c \phi_{\mathrm{U}}
\end{array}\right\| \\
& \mathrm{d}_{\mathrm{PQ}_{\mathrm{D}}}=\sqrt{\begin{array}{l}
\left(x_{\mathrm{S}}+s_{\mathrm{Sx}}^{\prime \mathrm{Q}} c \phi_{\mathrm{S}}-s_{\mathrm{Sy}}^{\prime \mathrm{Q}} s \phi_{\mathrm{S}}-x_{\mathrm{U}}-s_{\mathrm{U} x}^{\prime \mathrm{B}} c \phi_{\mathrm{U}}+s_{\mathrm{Uy}}^{\prime \mathrm{P}} s \phi_{\mathrm{U}}\right)^{2}+ \\
\left(y_{\mathrm{S}}+s_{\mathrm{Sx}}^{\prime \mathrm{Q}} s \phi_{\mathrm{S}}+s_{\mathrm{Sy}}^{\prime \mathrm{Q}} c \phi_{\mathrm{S}}-y_{\mathrm{U}}-s_{\mathrm{U} x}^{\prime \mathrm{B}} s \phi_{\mathrm{U}}-s_{\mathrm{Uy}}^{\prime \mathrm{P}} c \phi_{\mathrm{U}}\right)^{2}
\end{array}}
\end{aligned}
$$

Thus the primary TSD compression is:

$$
\begin{aligned}
& \Delta \mathrm{d}_{\mathrm{PQ}}=\text { Initial length }- \text { Displaced length } \\
& \Delta \mathrm{d}_{\mathrm{PQ}}=\mathrm{d}_{\mathrm{PQ}_{\mathrm{I}}}-\sqrt{\begin{array}{l}
\left(x_{\mathrm{S}}+s_{\mathrm{Sx}}^{\prime \mathrm{Q}} c \phi_{\mathrm{S}}-s_{\mathrm{Sy}}^{\prime \mathrm{Q}} s \phi_{\mathrm{S}}-x_{\mathrm{U}}-s_{\mathrm{Ux}}^{\prime \mathrm{B}} c \phi_{\mathrm{U}}+s_{\mathrm{Uy}}^{\prime \mathrm{P}} s \phi_{\mathrm{U}}\right)^{2}+ \\
\left(y_{\mathrm{S}}+s_{\mathrm{Sx}}^{\prime \mathrm{Q}} s \phi_{\mathrm{S}}+s_{\mathrm{Sy}}^{\prime \mathrm{Q}} c \phi_{\mathrm{S}}-y_{\mathrm{U}}-s_{\mathrm{Ux}}^{\prime \mathrm{B}} s \phi_{\mathrm{U}}-s_{\mathrm{Uy}}^{\prime \mathrm{P}} c \phi_{\mathrm{U}}\right)^{2}
\end{array}}
\end{aligned}
$$

The first time derivative of vector $\mathbf{d}_{\mathrm{PQ}}$ is the primary TSD's compression velocity vector:

$$
\dot{\mathbf{d}}_{\mathrm{PQ}}=\left[\begin{array}{l}
\dot{x}_{\mathrm{S}}-\dot{\phi}_{\mathrm{S}} s_{\mathrm{Sx}}^{\prime \mathrm{Q}} s \phi_{\mathrm{S}}-\dot{\phi}_{\mathrm{S}} s_{\mathrm{Sy}}^{\prime \mathrm{Q}} c \phi_{\mathrm{S}}-\dot{x}_{\mathrm{U}}+\dot{\phi}_{\mathrm{U}} s_{\mathrm{Ux}}^{\prime \mathrm{B}} s \phi_{\mathrm{U}}+\dot{\phi}_{\mathrm{U}} s_{\mathrm{Uy}}^{\prime \mathrm{P}} c \phi_{\mathrm{U}} \\
\dot{y}_{\mathrm{S}}+\dot{\phi}_{\mathrm{S}} s_{\mathrm{Sx}}^{\prime \mathrm{Q}} c \phi_{\mathrm{S}}-\dot{\phi}_{\mathrm{S}} s_{\mathrm{Sy}}^{\prime \mathrm{Q}} s \phi_{\mathrm{S}}-\dot{y}_{\mathrm{U}}-\dot{\phi}_{\mathrm{U}} s_{\mathrm{Ux}}^{\mathrm{B}} c \phi_{\mathrm{U}}+\dot{\phi}_{\mathrm{U}} s_{\mathrm{Uy}}^{\prime \mathrm{P}} s \phi_{\mathrm{U}}
\end{array}\right]
$$

By multiplying the magnitude of this vector by SIGN the compressive velocity can be written as the following scalar:

$$
\begin{aligned}
& \Delta \dot{\mathrm{d}}_{\mathrm{PQ}}=\mathbf{S I G N} \times\left\|\dot{\mathbf{d}}_{\mathrm{PQ}}\right\| \\
& \Delta \dot{\mathrm{d}}_{\mathrm{PQ}}=\mathbf{S I G N} \times \sqrt{\begin{array}{l}
\left(\dot{x}_{\mathrm{S}}-\dot{\phi}_{\mathrm{S}} s_{\mathrm{Sx}}^{\prime \mathrm{Q}} s \phi_{\mathrm{S}}-\dot{\phi}_{\mathrm{S}} s_{\mathrm{Sy}}^{\prime \mathrm{Q}} c \phi_{\mathrm{S}}-\dot{x}_{\mathrm{U}}+\dot{\phi}_{\mathrm{U}} s_{\mathrm{Ux}}^{\prime \mathrm{B}} s \phi_{\mathrm{U}}+\dot{\phi}_{\mathrm{U}} s_{\mathrm{Uy}}^{\mathrm{P}} c \phi_{\mathrm{U}}\right)^{2}+ \\
\left(\dot{y}_{\mathrm{S}}+\dot{\phi}_{\mathrm{S}} s_{\mathrm{Sx}}^{\prime \mathrm{Q}} c \phi_{\mathrm{S}}-\dot{\phi}_{\mathrm{S}} s_{\mathrm{Sy}}^{\prime \mathrm{Q}} s \phi_{\mathrm{S}}-\dot{y}_{\mathrm{U}}-\dot{\phi}_{\mathrm{U}} s_{\mathrm{U} x}^{\prime \mathrm{B}} c \phi_{\mathrm{U}}+\dot{\phi}_{\mathrm{U}} s_{\mathrm{Uy}}^{\prime \mathrm{P}} s \phi_{\mathrm{U}}\right)^{2}
\end{array}}
\end{aligned}
$$

SIGN is positive when the TSD is compressing (that is, when the current TSD length is less than the previous). This SIGN convention supports the assumption that a positive TSD damper force is an outward force from its center towards points Q and P. 
The outward primary TSD force is the sum of its spring and damper forces.

$$
\begin{aligned}
& \mathrm{F}^{\mathrm{P}}=\text { Primary Spring Force }+ \text { Primary Damper Force } \\
& \mathrm{F}^{\mathrm{P}}=\mathrm{k}_{\mathrm{p}} \Delta \mathrm{d}_{\mathrm{PQ}}+\mathbf{S I G N} \times \mathrm{n}_{\mathrm{p}} \Delta \dot{\mathrm{d}}_{\mathrm{PQ}}
\end{aligned}
$$

\subsubsection{Tire TSD}

This section develops the force created by the tire TSD. The tire TSD, shown in Figure $6-9$, produces a force $\mathrm{F}^{\mathrm{T}}$ in the global $\mathrm{Y}$-direction in proportion to its compression $\Delta \mathrm{d}_{\mathrm{AU}}^{\mathrm{y}}$ and compression velocity $\Delta \dot{\mathrm{d}}_{\mathrm{AU}}^{\mathrm{y}}$. The y-superscript on the compression and compression velocity serve to emphasize that, unlike the primary TSD, both are directed in the global $\mathrm{Y}$-direction.

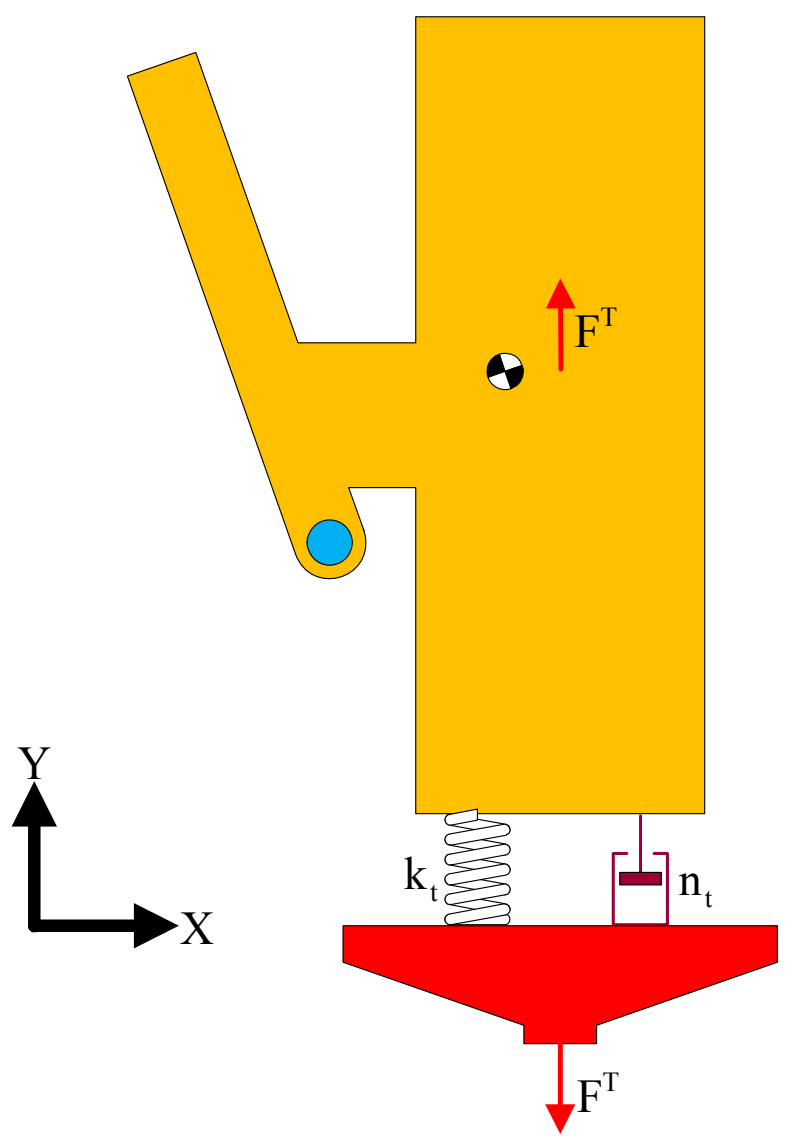

Figure 6-9 Non-Linear Model Tire TSD 
To specify the tire TSD compression, its initial and displaced length must be specified. The initial length, $\mathrm{d}_{\mathrm{AU}_{\mathrm{I}}}^{\mathrm{y}}$, is its length without any load on it, or free length. The displaced length is the global unsprung mass Y-position minus the actuator Y-position. Thus, the tire TSD compression is:

$$
\begin{aligned}
& \Delta \mathrm{d}_{\mathrm{AU}}^{\mathrm{y}}=\text { Initial length }- \text { Displaced length } \\
& \Delta \mathrm{d}_{\mathrm{AU}}^{\mathrm{y}}=\mathrm{d}_{\mathrm{AU}_{\mathrm{I}}}^{\mathrm{y}}-\left(\mathrm{y}_{\mathrm{U}}-\mathrm{y}_{\mathrm{A}}\right)
\end{aligned}
$$

Positive spring forces are applied outward from the TSD's center towards the unsprung mass and actuator when the spring is compressed.

The first time derivative of tire TSD compression is the tire TSD compression velocity:

$$
\Delta \dot{\mathrm{d}}_{\mathrm{AU}}^{\mathrm{y}}=-\dot{\mathrm{y}}_{\mathrm{U}}+\dot{\mathrm{y}}_{\mathrm{A}}
$$

Positive damper forces are applied outward from the TSD's center toward the unsprung mass and actuator when the damper is compressing. The red vectors in Figure 6-9 show the positive tire TSD force direction. The outward tire TSD force is the sum of its spring and damper forces:

$$
\begin{aligned}
& \mathrm{F}^{\mathrm{T}}=\text { Tire Spring Force }+ \text { Tire Damper Force } \\
& \mathrm{F}^{\mathrm{T}}=\mathrm{k}_{\mathrm{t}} \Delta \mathrm{d}_{\mathrm{AU}}^{\mathrm{y}}+\mathrm{n}_{\mathrm{t}} \Delta \dot{\mathrm{d}}_{\mathrm{AU}}^{\mathrm{y}}
\end{aligned}
$$

\subsubsection{Sprung Mass Forces}

This section identifies the applied sprung mass forces. Figure 6-10 shows the location of these forces on the sprung mass. The forces acting on this body are due to the primary TSD and gravity. 


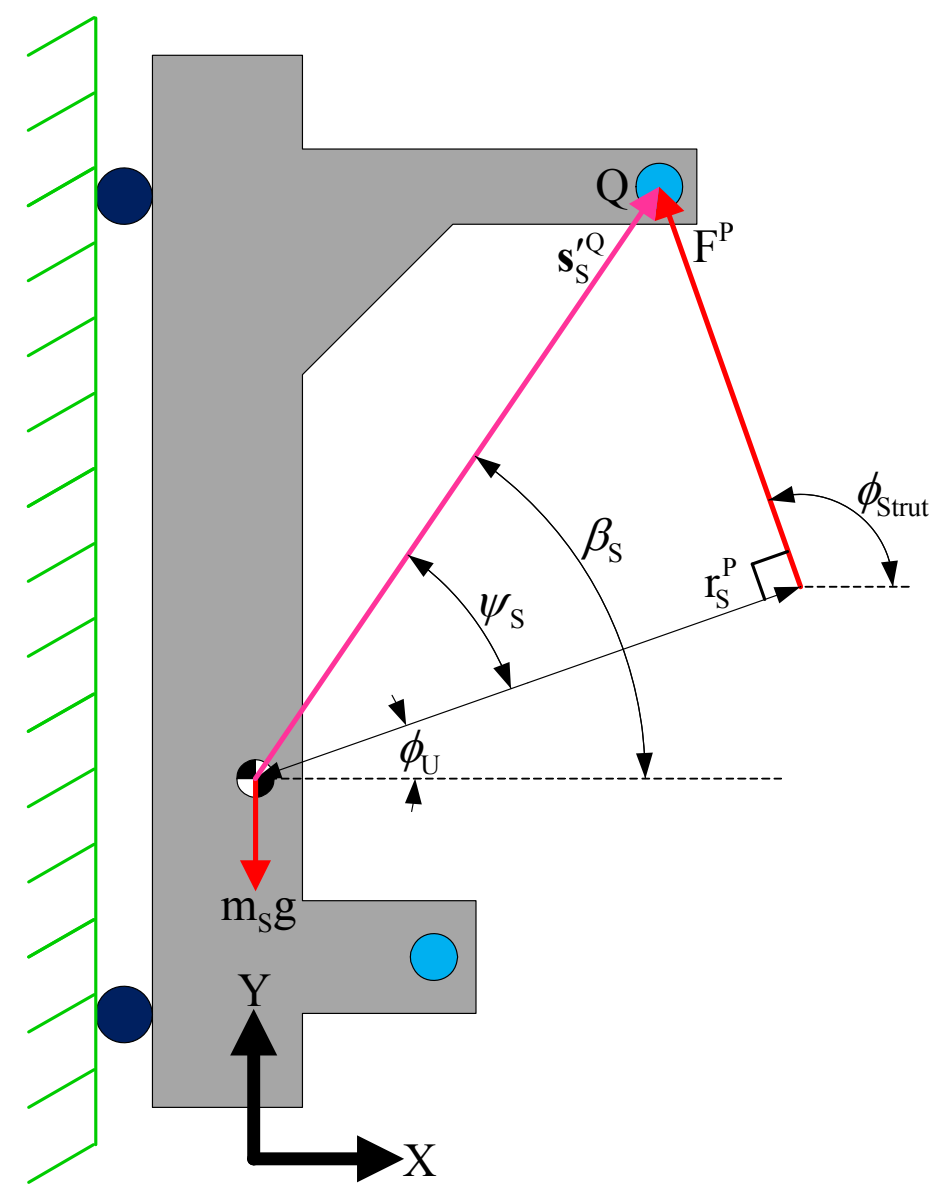

Figure 6-10 Non-Linear Model Sprung Mass Forces

In this Figure $\phi_{\text {Strut }}$ is the angle between the global X-axis and the strut's translation line:

$$
\phi_{\mathrm{Strut}}=\phi_{\mathrm{U}}+90^{\circ}
$$

where angle $\phi_{\mathrm{U}}$ is the unsprung mass's angular coordinate. Fixed angle $\beta_{\mathrm{S}}$ is measured between the global $\mathrm{X}$-axis and vector, $\mathbf{s}_{\mathrm{S}}^{\mathrm{Q}}$. The angle $\psi_{\mathrm{S}}$ :

$$
\psi_{\mathrm{S}}=\beta_{\mathrm{S}}-\phi_{\mathrm{U}}
$$

is used to define the moment arm $r_{S}^{P}$ perpendicular to $F^{P}$. This moment arm is the length of the line from the sprung mass $\mathrm{CG}$ to $\mathrm{F}^{\mathrm{P}}$ : 


$$
\mathrm{r}_{\mathrm{S}}^{\mathrm{P}}=\left\|\mathbf{s}_{\mathrm{S}}^{\prime \mathrm{Q}}\right\| \cos \psi_{\mathrm{S}}=\sqrt{\left(\mathrm{s}_{\mathrm{Sx}}^{\prime \mathrm{Q}}\right)^{2}+\left(\mathrm{s}_{\mathrm{Sy}}^{\prime \mathrm{Q}}\right)^{2}} \cos \psi_{\mathrm{S}}
$$

The sprung mass and gravitational acceleration are $\mathrm{m}_{\mathrm{s}}$ and $\mathrm{g}$, respectively. With these variables defined, the sums of forces applied to the sprung mass in the global $\mathrm{X}$ and $\mathrm{Y}$ directions are:

$$
\begin{aligned}
& \sum \mathrm{F}_{\mathrm{Sx} \rightarrow}^{\mathrm{A}}=\mathrm{F}^{\mathrm{P}} \cos \phi_{\text {Strut }} \\
& \sum \mathrm{F}_{\mathrm{Sy} \uparrow}^{\mathrm{A}}=\mathrm{F}^{\mathrm{P}} \sin \phi_{\text {Strut }}-\mathrm{m}_{\mathrm{S}} \mathrm{g}
\end{aligned}
$$

The sum of the counter-clockwise torque applied about the sprung mass CG is:

$$
\sum \tau_{\mathrm{S} U}^{\mathrm{A}}=\mathrm{r}_{\mathrm{S}}^{\mathrm{P}} \times \mathrm{F}^{\mathrm{P}}
$$

The sprung mass force vector is:

$$
\mathbf{F}_{\mathrm{S}}^{\mathrm{A}}=\left[\begin{array}{c}
\mathrm{X} \text {-Force } \\
\text { Y-Force } \\
\text { CC-Torque }
\end{array}\right]=\left[\begin{array}{c}
\sum \mathrm{F}_{\mathrm{Sx} \rightarrow}^{\mathrm{A}} \\
\sum \mathrm{F}_{\mathrm{Sy} \uparrow}^{\mathrm{A}} \\
\sum \tau_{\mathrm{SU}}^{\mathrm{A}}
\end{array}\right]=\left[\begin{array}{c}
\mathrm{F}^{\mathrm{P}} \cos \phi_{\mathrm{Strut}} \\
\mathrm{F}^{\mathrm{P}} \sin \phi_{\text {Strut }}-\mathrm{m}_{\mathrm{S}} \mathrm{g} \\
\mathrm{r}_{\mathrm{S}}^{\mathrm{P}} \times \mathrm{F}^{\mathrm{P}}
\end{array}\right]
$$

The sprung mass force vector can be simplified as follows:

$$
\mathbf{F}_{\mathrm{S}}^{\mathrm{A}}=\left[\begin{array}{c}
-\mathrm{F}^{\mathrm{P}} \sin \phi_{\mathrm{U}} \\
\mathrm{F}^{\mathrm{P}} \cos \phi_{\mathrm{U}}-\mathrm{m}_{\mathrm{S}} \mathrm{g} \\
\mathrm{r}_{\mathrm{S}}^{\mathrm{P}} \times \mathrm{F}^{\mathrm{P}}
\end{array}\right]
$$

by noting the trigonometric relation: 


$$
\begin{aligned}
& \cos \left(\phi+90^{\circ}\right)=-\sin \phi \\
& \sin \left(\phi+90^{\circ}\right)=\cos \phi
\end{aligned}
$$

\subsubsection{Control Arm Forces}

This section identifies the applied control arm force. Figure 6-11 shows the location of the applied force acting on the control arm.

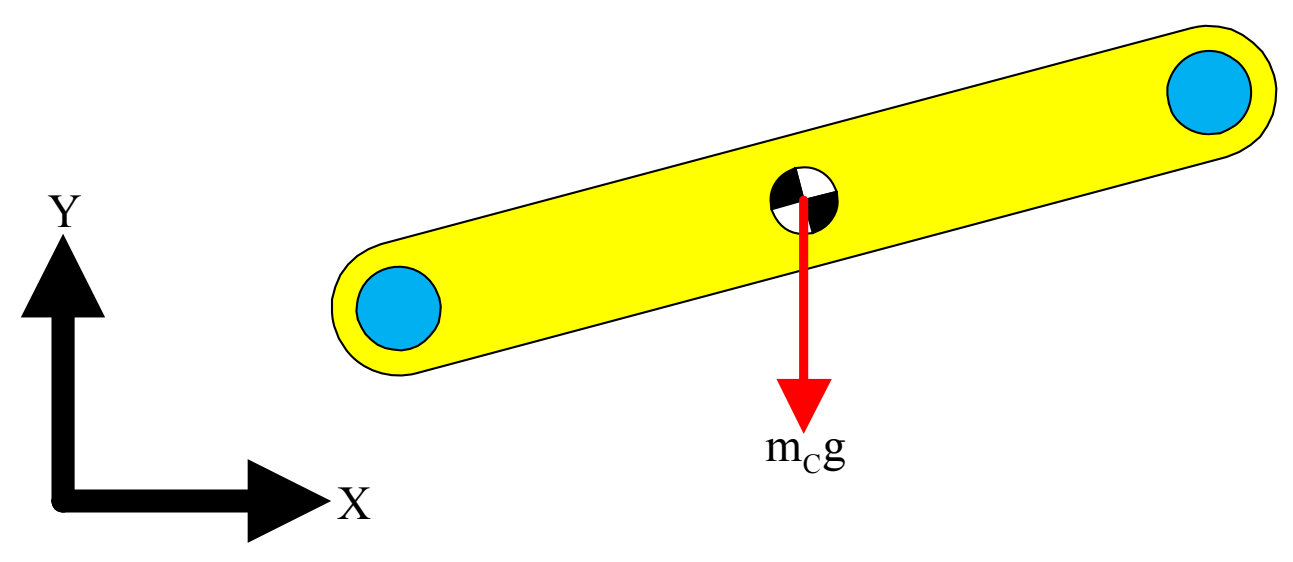

Figure 6-11 Non-Linear Model Control Arm Force

In this Figure, the control arm mass and gravitational acceleration are $\mathrm{m}_{\mathrm{C}}$ and $\mathrm{g}$, respectively. The only applied force acting on the control arm is its gravitational force. Therefore the sums of forces applied to the control arm in the global $\mathrm{X}$ and $\mathrm{Y}$-directions are:

$$
\begin{aligned}
& \sum \mathrm{F}_{\mathrm{Cx} \rightarrow}^{\mathrm{A}}=0 \\
& \sum \mathrm{F}_{\mathrm{Cy} \uparrow}^{\mathrm{A}}=-\mathrm{m}_{\mathrm{C}} \mathrm{g}
\end{aligned}
$$

The sum of the counter-clockwise torque applied about the control arm CG is:

$$
\sum \tau_{\mathrm{C} \cup}^{\mathrm{A}}=0
$$


The control arm force vector is:

$$
\mathbf{F}_{\mathrm{C}}^{\mathrm{A}}=\left[\begin{array}{c}
\mathrm{X} \text {-Force } \\
\text { Y-Force } \\
\text { CC-Torque }
\end{array}\right]=\left[\begin{array}{c}
\sum \mathrm{F}_{\mathrm{Cx} \rightarrow}^{\mathrm{A}} \\
\sum \mathrm{F}_{\mathrm{Cy} \uparrow}^{\mathrm{A}} \\
\sum \tau_{\mathrm{C} \cup}^{\mathrm{A}}
\end{array}\right]=\left[\begin{array}{c}
0 \\
-\mathrm{m}_{\mathrm{C}} \mathrm{g} \\
0
\end{array}\right]
$$

\subsubsection{Unsprung Mass Forces}

This section identifies the applied unsprung mass forces. Figure 6-12 shows the location of these forces on the unsprung mass. The forces acting on this body are due to the primary and tire TSDs and gravity.

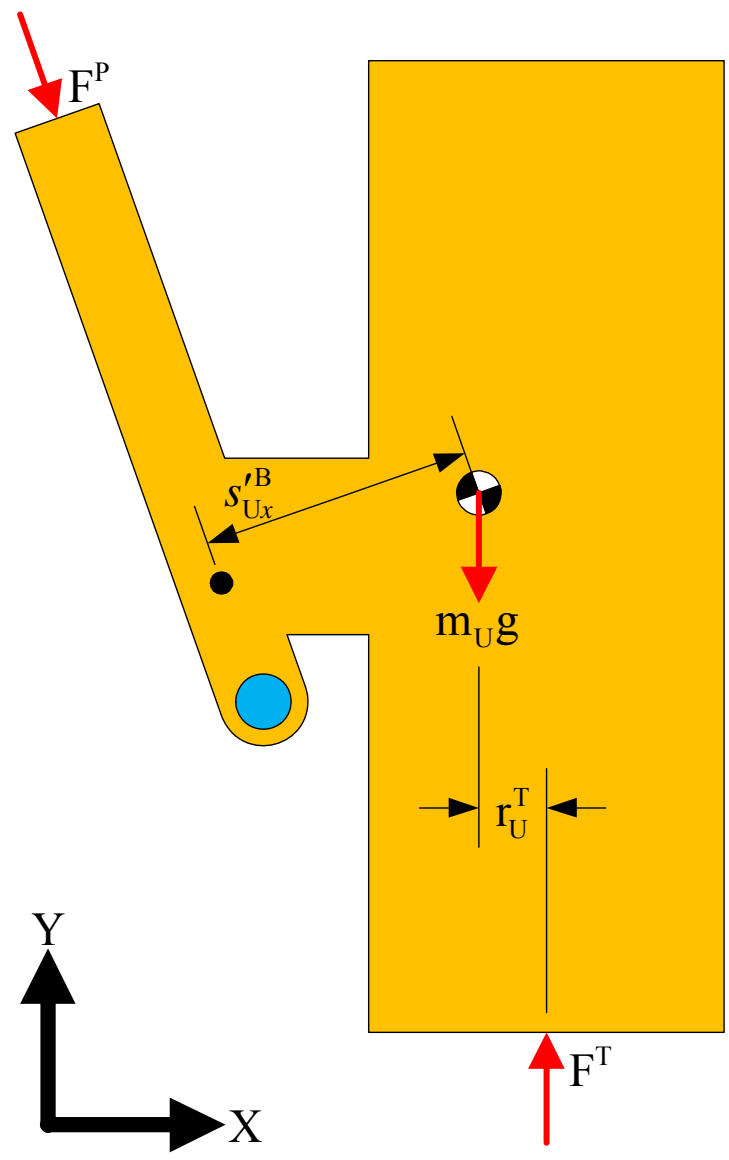

Figure 6-12 Non-Linear Model Unsprung Mass Forces 
In this Figure, $r_{U}^{T}$ is the moment arm perpendicular to the tire force vector $F^{T}$. This moment arm is the length of the line from the unsprung mass $C G$ to $F^{T}$.

Likewise, $s_{U x}^{\prime B}$ is the moment arm perpendicular to the primary TSD force vector $\mathrm{F}^{\mathrm{P}}$. This moment arm is the length of the line from the unsprung mass CG to $\mathrm{F}^{\mathrm{P}}$.

The unsprung mass and gravitational acceleration are $\mathrm{m}_{\mathrm{U}}$ and $\mathrm{g}$, respectively. The last variable to recall is $\phi_{\text {Strut }}$ from Figure 6-10:

$$
\phi_{\mathrm{Strut}}=\phi_{\mathrm{U}}+90^{\circ}
$$

where angle $\phi_{\mathrm{U}}$ is the unsprung mass's angular coordinate.

With these variables defined and noting the trigonometric relations used to simplify $\phi_{\text {Strut }}$, the sums of forces applied to the unsprung mass in the global $\mathrm{X}$ and $\mathrm{Y}$-directions are:

$$
\begin{array}{ll}
\sum \mathrm{F}_{\mathrm{Ux} \rightarrow}^{\mathrm{A}}=-\mathrm{F}^{\mathrm{P}} \cos \phi_{\text {Strut }} & =\mathrm{F}^{\mathrm{P}} \sin \phi_{\mathrm{U}} \\
\sum \mathrm{F}_{\mathrm{Uy} \uparrow}^{\mathrm{A}}=-\mathrm{F}^{\mathrm{P}} \sin \phi_{\text {Strut }}-\mathrm{m}_{\mathrm{U}} \mathrm{g}+\mathrm{F}^{\mathrm{T}}=-\mathrm{F}^{\mathrm{P}} \cos \phi_{\mathrm{U}}-\mathrm{m}_{\mathrm{U}} \mathrm{g}+\mathrm{F}^{\mathrm{T}}
\end{array}
$$

The sum of the counter-clockwise torque applied about the unsprung mass CG is:

$$
\sum \tau_{\mathrm{UU}}^{\mathrm{A}}={s_{\mathrm{Ux}}^{\prime \mathrm{B}}}^{\mathrm{P}} \times \mathrm{F}^{\mathrm{P}}+\mathrm{r}_{\mathrm{U}}^{\mathrm{T}} \times \mathrm{F}^{\mathrm{T}}
$$

The unsprung mass force vector is:

$$
\mathbf{F}_{\mathrm{U}}^{\mathrm{A}}=\left[\begin{array}{c}
\mathrm{X} \text {-Force } \\
\text { Y-Force } \\
\text { CC-Torque }
\end{array}\right]=\left[\begin{array}{c}
\sum \mathrm{F}_{\mathrm{Ux} \rightarrow}^{\mathrm{A}} \\
\sum \mathrm{F}_{\mathrm{Uy} \uparrow}^{\mathrm{A}} \\
\sum \tau_{\mathrm{U} U}^{\mathrm{A}}
\end{array}\right]=\left[\begin{array}{c}
\mathrm{F}^{\mathrm{P}} \sin \phi_{\mathrm{U}} \\
-\mathrm{F}^{\mathrm{P}} \cos \phi_{\mathrm{U}}-\mathrm{m}_{\mathrm{U}} \mathrm{g}+\mathrm{F}^{\mathrm{T}} \\
\mathrm{s}_{\mathrm{Ux}}^{\mathrm{B}} \times \mathrm{F}^{\mathrm{P}}+\mathrm{r}_{\mathrm{U}}^{\mathrm{T}} \times \mathrm{F}^{\mathrm{T}}
\end{array}\right]
$$




\subsubsection{Complete Force Vector}

The complete force vector $\mathbf{Q}^{\mathbf{A}}$ is populated from each body's individual force vector:

$$
\mathbf{Q}^{\mathrm{A}}=\left[\begin{array}{c}
\mathbf{F}_{\mathrm{S}}^{\mathrm{A}} \\
\mathbf{F}_{\mathrm{C}}^{\mathrm{A}} \\
\mathbf{F}_{\mathrm{U}}^{\mathrm{A}} \\
\mathrm{F}_{\mathrm{A}}^{\mathrm{A}}
\end{array}\right]=\left[\begin{array}{c}
\sum \mathrm{F}_{\mathrm{Sx} \rightarrow}^{\mathrm{A}} \\
\sum \mathrm{F}_{\mathrm{Sy} \uparrow}^{\mathrm{A}} \\
\sum \tau_{\mathrm{SU}}^{\mathrm{A}} \\
\sum \mathrm{F}_{\mathrm{Cx} \rightarrow}^{\mathrm{A}} \\
\sum \mathrm{F}_{\mathrm{Cy} \uparrow}^{\mathrm{A}} \\
\sum \tau_{\mathrm{CU}}^{\mathrm{A}} \\
\sum \mathrm{F}_{\mathrm{Ux} \rightarrow}^{\mathrm{A}} \\
\sum \mathrm{F}_{\mathrm{Uy} \uparrow}^{\mathrm{A}} \\
\sum \tau_{\mathrm{UU}}^{\mathrm{A}} \\
\sum \mathrm{F}_{\mathrm{Ay} \uparrow}^{\mathrm{A}}
\end{array}\right]=\left[\begin{array}{c}
-\mathrm{F}^{\mathrm{P}} \sin \phi_{\mathrm{U}} \\
\mathrm{F}^{\mathrm{P}} \cos \phi_{\mathrm{U}}-\mathrm{m}_{\mathrm{S}} \mathrm{g} \\
\mathrm{r}_{\mathrm{S}}^{\mathrm{P}} \times \mathrm{F}^{\mathrm{P}} \\
0 \\
-\mathrm{m}_{\mathrm{C}} \mathrm{g} \\
0 \\
\mathrm{~F}^{\mathrm{P}} \sin \phi_{\mathrm{U}} \\
-\mathrm{F}^{\mathrm{P}} \cos \phi_{\mathrm{U}}-\mathrm{m}_{\mathrm{U}} \mathrm{g}+\mathrm{F}^{\mathrm{T}} \\
\mathrm{s}_{\mathrm{Ux}}^{\mathrm{B}} \times \mathrm{F}^{\mathrm{P}}+\mathrm{r}_{\mathrm{U}}^{\mathrm{T}} \times \mathrm{F}^{\mathrm{T}} \\
0 \\
0
\end{array}\right]
$$

The applied actuator force is set to zero because it has no effect on the dynamics of interest. 


\subsection{Mass Matrix}

The mass matrix for this model has the form:

$$
\mathbf{M}=\left[\begin{array}{cccccccccc}
\mathrm{m}_{\mathrm{S}} & 0 & 0 & 0 & 0 & 0 & 0 & 0 & 0 & 0 \\
0 & \mathrm{~m}_{\mathrm{S}} & 0 & 0 & 0 & 0 & 0 & 0 & 0 & 0 \\
0 & 0 & j_{\mathrm{S}} & 0 & 0 & 0 & 0 & 0 & 0 & 0 \\
0 & 0 & 0 & \mathrm{~m}_{\mathrm{C}} & 0 & 0 & 0 & 0 & 0 & 0 \\
0 & 0 & 0 & 0 & \mathrm{~m}_{\mathrm{C}} & 0 & 0 & 0 & 0 & 0 \\
0 & 0 & 0 & 0 & 0 & j_{\mathrm{C}} & 0 & 0 & 0 & 0 \\
0 & 0 & 0 & 0 & 0 & 0 & \mathrm{~m}_{\mathrm{U}} & 0 & 0 & 0 \\
0 & 0 & 0 & 0 & 0 & 0 & 0 & \mathrm{~m}_{\mathrm{U}} & 0 & 0 \\
0 & 0 & 0 & 0 & 0 & 0 & 0 & 0 & j_{\mathrm{U}} & 0 \\
0 & 0 & 0 & 0 & 0 & 0 & 0 & 0 & 0 & \mathrm{~m}_{\mathrm{A}}
\end{array}\right]
$$

where $\mathrm{m}_{\mathrm{S}}, \mathrm{m}_{\mathrm{C}}, \mathrm{m}_{\mathrm{U}}$, and $\mathrm{m}_{\mathrm{A}}$ are the sprung, control arm, unsprung, and actuator masses and $j_{\mathrm{S}}, j_{\mathrm{C}}$, and $j_{\mathrm{U}}$ are their respective moments of inertia. The sprung mass and inertia are derived from the sprung mass plate, adapter plate, strut tower, and ballast weights. The unsprung mass and inertia are derived from the springs, damper, upright, brake disk, wheel, and tire. The control arm's mass and inertia are derived from the two-piece control arm and tie rod. The actuator mass is considered negligible in this study and approximated as zero. For completeness and for easy adjustment of the model in the future, the dynamic equation associated with $\mathrm{m}_{\mathrm{A}}$ has been kept in the system of equations.

\subsection{McPherson Strut Non-Linear DAE}

With all the components of the DAE determined, it can be assembled. The DAE used to simulate the non-linear quarter-car's dynamic response is: 


$$
\begin{aligned}
& {\left[\begin{array}{cc}
\mathbf{M} & \boldsymbol{\Phi}_{\mathrm{q}}^{\mathrm{T}} \\
\boldsymbol{\Phi}_{\mathrm{q}} & \mathbf{0}
\end{array}\right]\left[\begin{array}{c}
\ddot{\mathbf{q}} \\
\lambda
\end{array}\right]=\left[\begin{array}{c}
\mathbf{Q}^{\mathbf{A}} \\
\boldsymbol{\alpha}
\end{array}\right]} \\
& {\left[\begin{array}{cccccccccccccccccc}
\mathrm{m}_{\mathrm{S}} & 0 & 0 & 0 & 0 & 0 & 0 & 0 & 0 & 0 & 0 & 1 & 0 & 0 & 0 & 0 & 0 & 0 \\
0 & \mathrm{~m}_{\mathrm{S}} & 0 & 0 & 0 & 0 & 0 & 0 & 0 & 0 & 0 & 0 & 0 & 1 & 0 & 0 & -s \phi_{\mathrm{U}} & 0 \\
0 & 0 & j_{\mathrm{S}} & 0 & 0 & 0 & 0 & 0 & 0 & 0 & 1 & 0 & 0 & 0 & 0 & 0 & 0 & 0 \\
0 & 0 & 0 & \mathrm{~m}_{\mathrm{C}} & 0 & 0 & 0 & 0 & 0 & 0 & 0 & 0 & -1 & 0 & 1 & 0 & 0 & 0 \\
0 & 0 & 0 & 0 & \mathrm{~m}_{\mathrm{C}} & 0 & 0 & 0 & 0 & 0 & 0 & 0 & 0 & -1 & 0 & 1 & 0 & 0 \\
0 & 0 & 0 & 0 & 0 & j_{\mathrm{C}} & 0 & 0 & 0 & 0 & 0 & 0 & \mathrm{es} \phi_{\mathrm{C}} & -\mathrm{ec} \phi_{\mathrm{C}} & -\mathrm{gs} \phi_{\mathrm{C}} & \mathrm{gc} \phi_{\mathrm{C}} & 0 & 0 \\
0 & 0 & 0 & 0 & 0 & 0 & \mathrm{~m}_{\mathrm{U}} & 0 & 0 & 0 & 0 & 0 & 0 & 0 & -1 & 0 & c \phi_{\mathrm{U}} & 0 \\
0 & 0 & 0 & 0 & 0 & 0 & 0 & \mathrm{~m}_{\mathrm{U}} & 0 & 0 & 0 & 0 & 0 & 0 & 0 & -1 & s \phi_{\mathrm{U}} & 0 \\
0 & 0 & 0 & 0 & 0 & 0 & 0 & 0 & j_{\mathrm{U}} & 0 & 0 & 0 & 0 & 0 & \mathrm{R}_{1} & \mathrm{R}_{2} & \mathrm{R}_{3} & 0 \\
0 & 0 & 0 & 0 & 0 & 0 & 0 & 0 & 0 & 0 & 0 & 0 & 0 & 0 & 0 & 0 & 0 & 1 \\
0 & 0 & 1 & 0 & 0 & 0 & 0 & 0 & 0 & 0 & 0 & 0 & 0 & 0 & 0 & 0 & 0 & 0 \\
1 & 0 & 0 & 0 & 0 & 0 & 0 & 0 & 0 & 0 & 0 & 0 & 0 & 0 & 0 & 0 & 0 & 0 \\
0 & 0 & 0 & -1 & 0 & \mathrm{es} \phi_{\mathrm{C}} & 0 & 0 & 0 & 0 & 0 & 0 & 0 & 0 & 0 & 0 & 0 & 0 \\
0 & 1 & 0 & 0 & -1 & -\mathrm{ec} \phi_{\mathrm{C}} & 0 & 0 & 0 & 0 & 0 & 0 & 0 & 0 & 0 & 0 & 0 & 0 \\
0 & 0 & 0 & 1 & 0 & -\mathrm{gs} \phi_{\mathrm{C}} & -1 & 0 & \mathrm{R}_{1} & 0 & 0 & 0 & 0 & 0 & 0 & 0 & 0 & 0 \\
0 & 0 & 0 & 0 & 1 & \mathrm{gc} \phi_{\mathrm{C}} & 0 & -1 & \mathrm{R}_{2} & 0 & 0 & 0 & 0 & 0 & 0 & 0 & 0 & 0 \\
0 & -\mathrm{s} \phi_{\mathrm{U}} & 0 & 0 & 0 & 0 & c \phi_{\mathrm{U}} & s \phi_{\mathrm{U}} & \mathrm{R}_{3} & 0 & 0 & 0 & 0 & 0 & 0 & 0 & 0 & 0 \\
0 & 0 & 0 & 0 & 0 & 0 & 0 & 0 & 0 & 1 & 0 & 0 & 0 & 0 & 0 & 0 & 0 & 0
\end{array}\right]\left[\begin{array}{c}
x_{\mathrm{S}} \\
y_{\mathrm{S}} \\
\phi_{\mathrm{S}} \\
x_{\mathrm{C}} \\
y_{\mathrm{C}} \\
\phi_{\mathrm{C}} \\
x_{\mathrm{U}} \\
y_{\mathrm{U}} \\
\phi_{\mathrm{U}} \\
y_{\mathrm{A}} \\
\lambda_{1} \\
\lambda_{2} \\
\lambda_{3} \\
\lambda_{4} \\
\lambda_{\mathrm{S}} \\
\lambda_{6} \\
\lambda_{7} \\
\lambda_{\mathrm{s}}
\end{array}\right]} \\
& =\left[\begin{array}{c}
-\mathrm{F}^{\mathrm{P}} \sin \phi_{\mathrm{U}} \\
\mathrm{F}^{\mathrm{P}} \cos \phi_{\mathrm{U}}-\mathrm{m}_{\mathrm{S}} \mathrm{g} \\
\mathrm{r}_{\mathrm{S}}^{\mathrm{P}} \times \mathrm{F}^{\mathrm{P}} \\
0 \\
-\mathrm{m}_{\mathrm{C}} \mathrm{g} \\
0 \\
\mathrm{~F}^{\mathrm{P}} \sin \phi_{\mathrm{U}} \\
-\mathrm{F}^{\mathrm{P}} \cos \phi_{\mathrm{U}}-\mathrm{m}_{\mathrm{U}} \mathrm{g}+\mathrm{F}^{\mathrm{T}} \\
\mathrm{s}_{\mathrm{Ux}}^{\prime \mathrm{B}} \times \mathrm{F}^{\mathrm{P}}+\mathrm{r}_{\mathrm{U}}^{\mathrm{T}} \times \mathrm{F}^{\mathrm{T}} \\
0 \\
0 \\
0 \\
-\mathrm{R}_{3} \\
-\mathrm{R}_{4} \\
-\mathrm{R}_{5} \\
-\mathrm{R}_{6} \\
-\mathrm{R}_{7} \\
-\frac{d^{2} f(t)}{d t^{2}}
\end{array}\right]
\end{aligned}
$$




\section{NON-LINEAR QUARTER-CAR MODEL SYSTEM IDENTIFICATION}

\subsection{Introduction}

This chapter presents the background and results of the system identification of the nonlinear quarter-car McPherson strut suspension model developed in Chapter 6. The purpose of this system identification is to determine model parameters that best facilitate the non-linear model's prediction of the experimental sprung and unsprung mass accelerations. Using these parameters with the non-linear model will create an optimal non-linear model that can be used for dynamic response prediction and control algorithm design.

\subsection{Simulation}

The non-linear simulation was performed with the same constants used with the linear simulation. For the HHT integrator, epsilon and alpha were set to $\varepsilon=10^{-4}$ and $\alpha=-1 / 3$. Likewise, the system identification process used was identical.

\subsection{Procedure}

The method used to perform system identification on the non-linear model is similar to that used with the linear model. The same comparison period, 3-8 seconds, was used to evaluate the cost function. Also, similar concepts were used for the selection of the upper and lower bounds. However, because more parameters were available, more parameters were optimized.

The optimization parameters selected for the non-linear model system identification include the sprung, unsprung, and control arm mass and inertia; the stiffness value, damping value, and initial length of the primary and tire TSDs; and the length of the moment arm between the unsprung and actuator bodies. As with the linear system identification, initial values for these parameters were gathered by direct measurement, calculation, or estimation. Figure 7-1 shows the initial parameter estimates that comprise 
the non-optimized parameters. Moment of inertia estimates for the control arm and unsprung mass are shown in Appendix A. The sprung mass moment of inertia was obtained from a solid model of the sprung mass. Figure 7-2 shows the initial positions and velocities. Figure 7-3 shows the non-linear model's geometric constants.

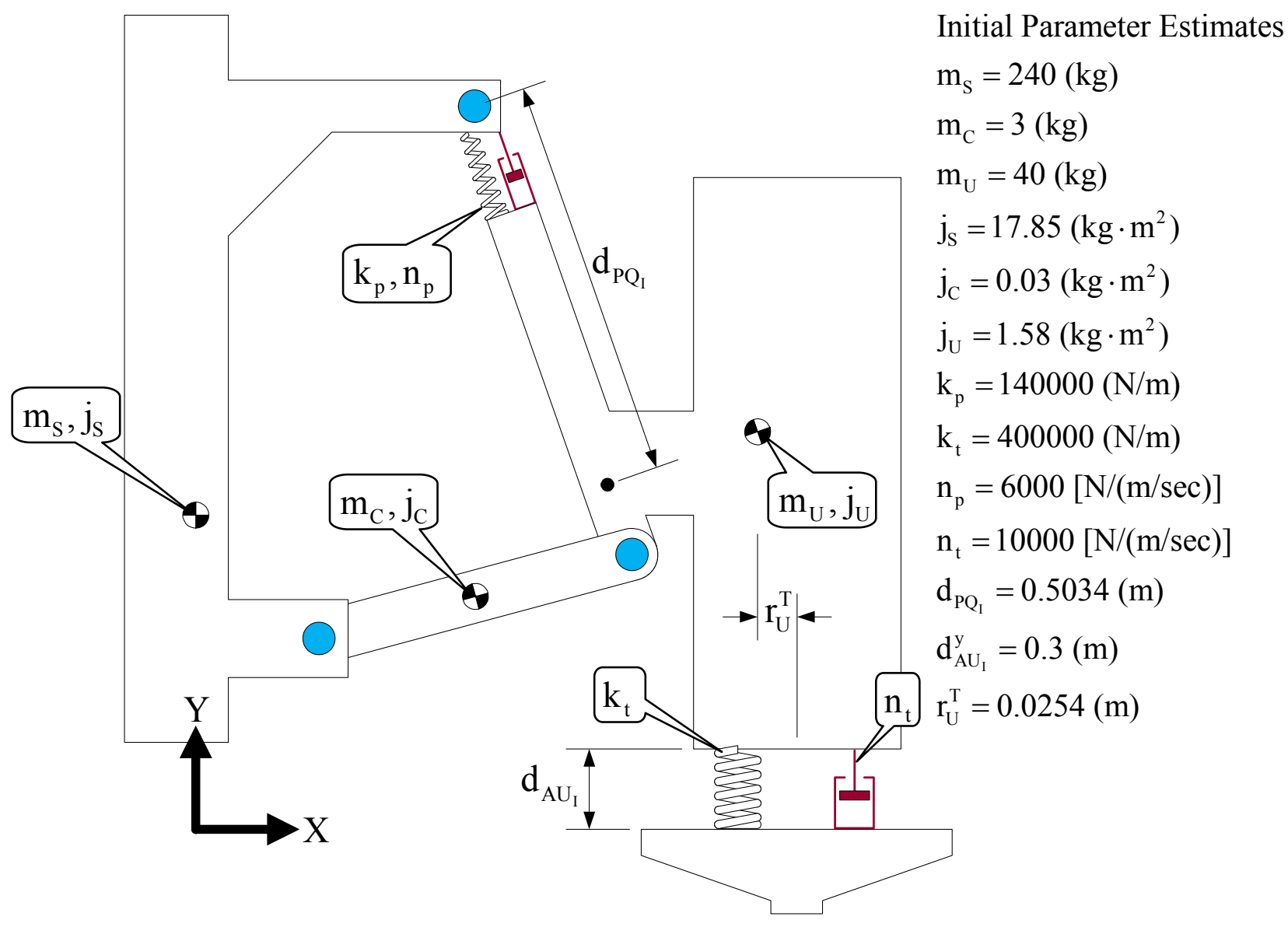

Figure 7-1 Non-Linear Model Initial Parameter Estimates 


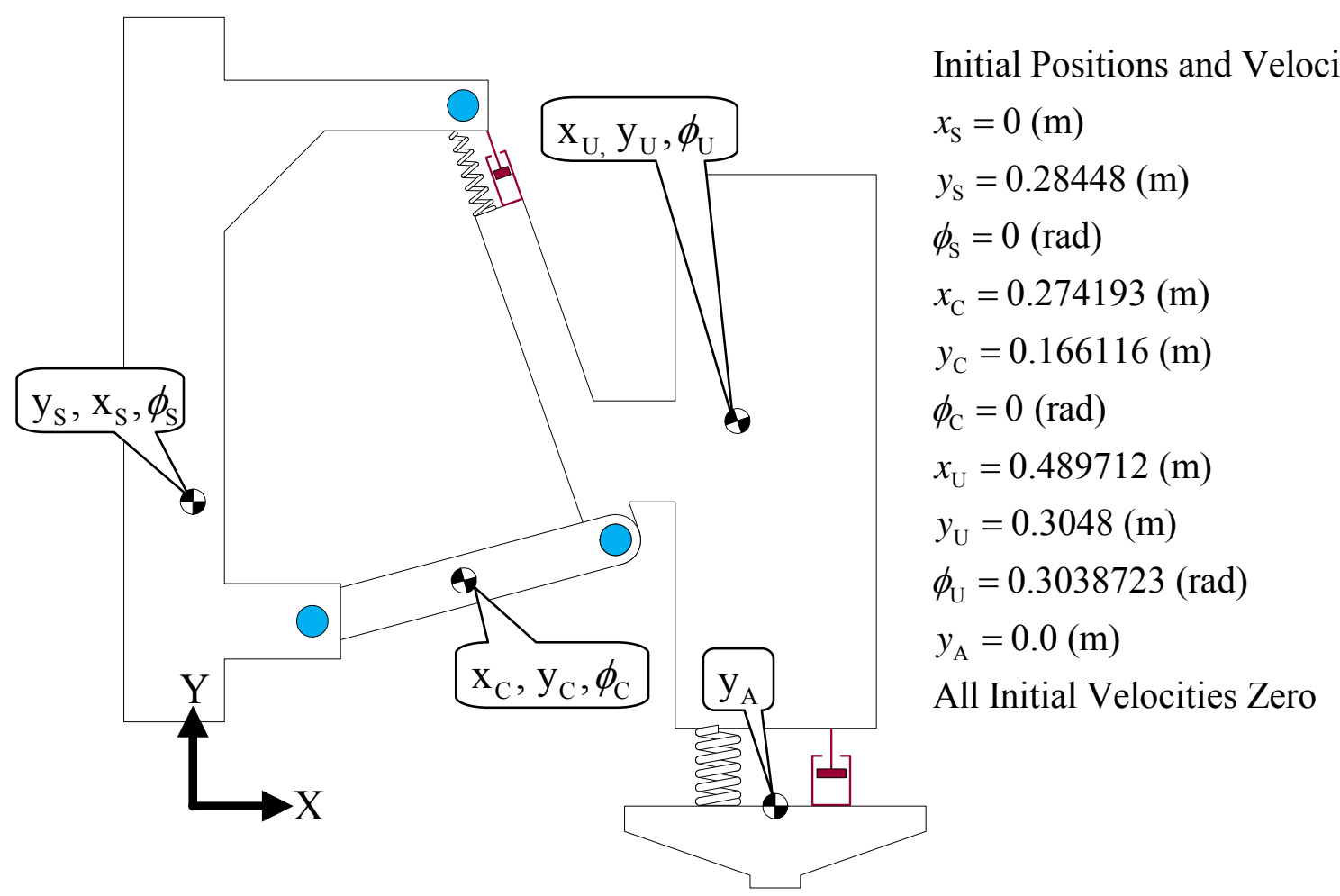

Figure 7-2 Non-Linear Model Initial Conditions

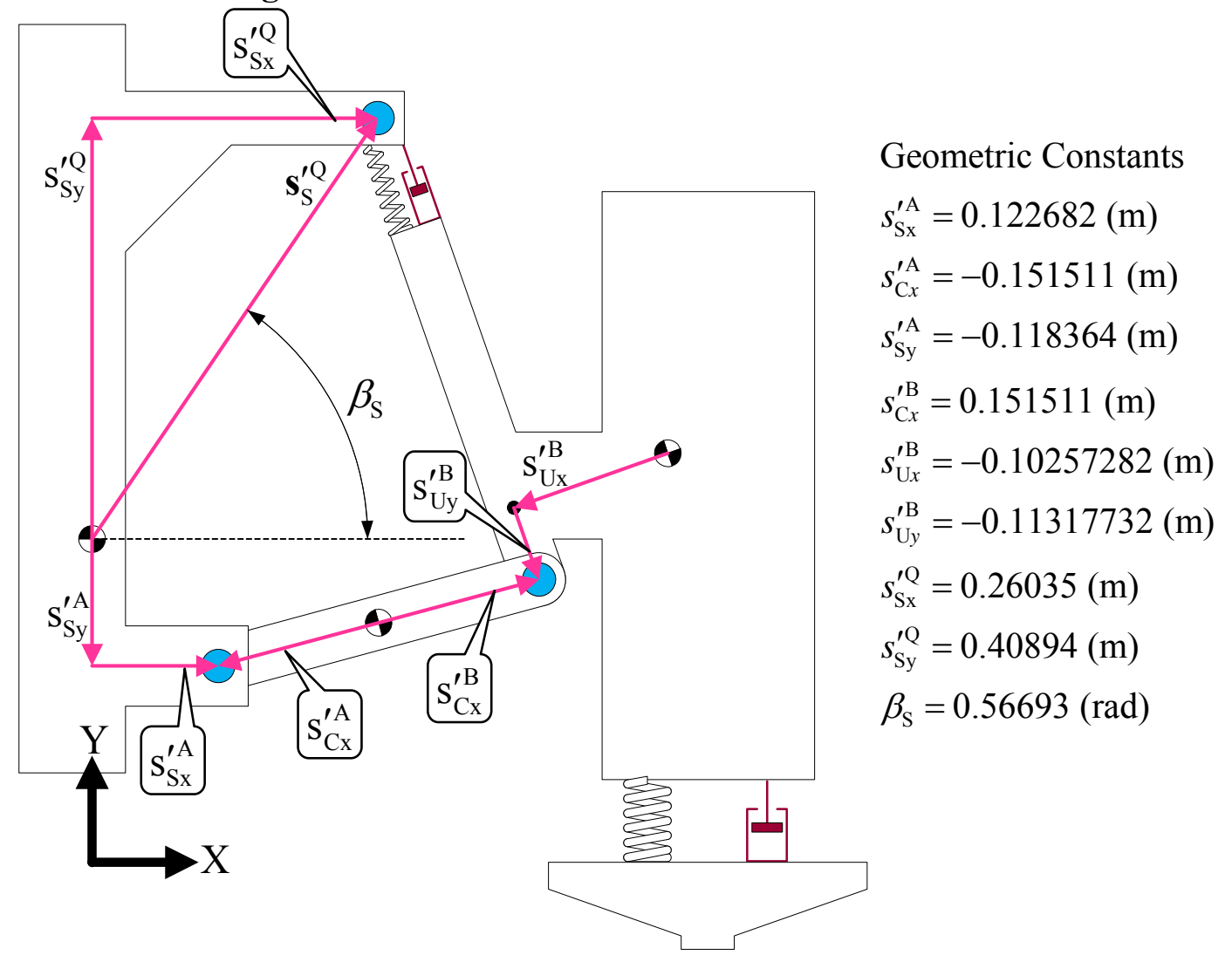

Figure 7-3 Non-Linear Model Geometric Constants 


\subsection{Performance Measure}

As with the linear model, the performance metric chosen to judge how well the mathematical model predicts the experimental acceleration is the ratio of the root-meansquare (RMS) acceleration error to the experimental RMS acceleration over the 3 to 50 second time interval. These ratios, for the sprung and unsprung mass, expressed in decibel form, are:

$$
\begin{aligned}
& \mathrm{R}_{\mathrm{S}}=20 \log 10\left(\frac{\operatorname{RMS}\left(e_{\mathrm{S}}\right)}{\operatorname{RMS}\left(\operatorname{Exp} \_\mathrm{a}_{\mathrm{S}}\right)}\right) \\
& \mathrm{R}_{\mathrm{U}}=20 \log 10\left(\frac{\operatorname{RMS}\left(e_{\mathrm{U}}\right)}{\operatorname{RMS}\left(\operatorname{Exp} \_\mathrm{a}_{\mathrm{U}}\right)}\right)
\end{aligned}
$$

where $R_{S}$ and $R_{U}$ are the sprung and unsprung mass performance ratios. As with the linear model, the lower the ratio, the better the performance.

\subsection{Non-Linear System Identification Results}

Figure 7-4 compares the acceleration predicted by the mathematical model (green dashed) using the initial parameter estimates with the experimentally measured accelerations (blue - solid). The error is highlighted in red (dash - dot). This nonoptimized model produces sprung and unsprung mass performance ratios of:

$$
\begin{aligned}
& R_{S}=-9.4216 d B \\
& R_{U}=-2.72 d B
\end{aligned}
$$

Figure 7-5 compares the acceleration predicted by the mathematical model using optimized parameters with the experimentally measured accelerations. This optimized model produces sprung and unsprung mass performance ratios of: 


$$
\begin{aligned}
& R_{S}=-18.447 d B \\
& R_{U}=-8.2972 d B
\end{aligned}
$$

Table 7-1 shows the optimal parameters for the non-linear model:
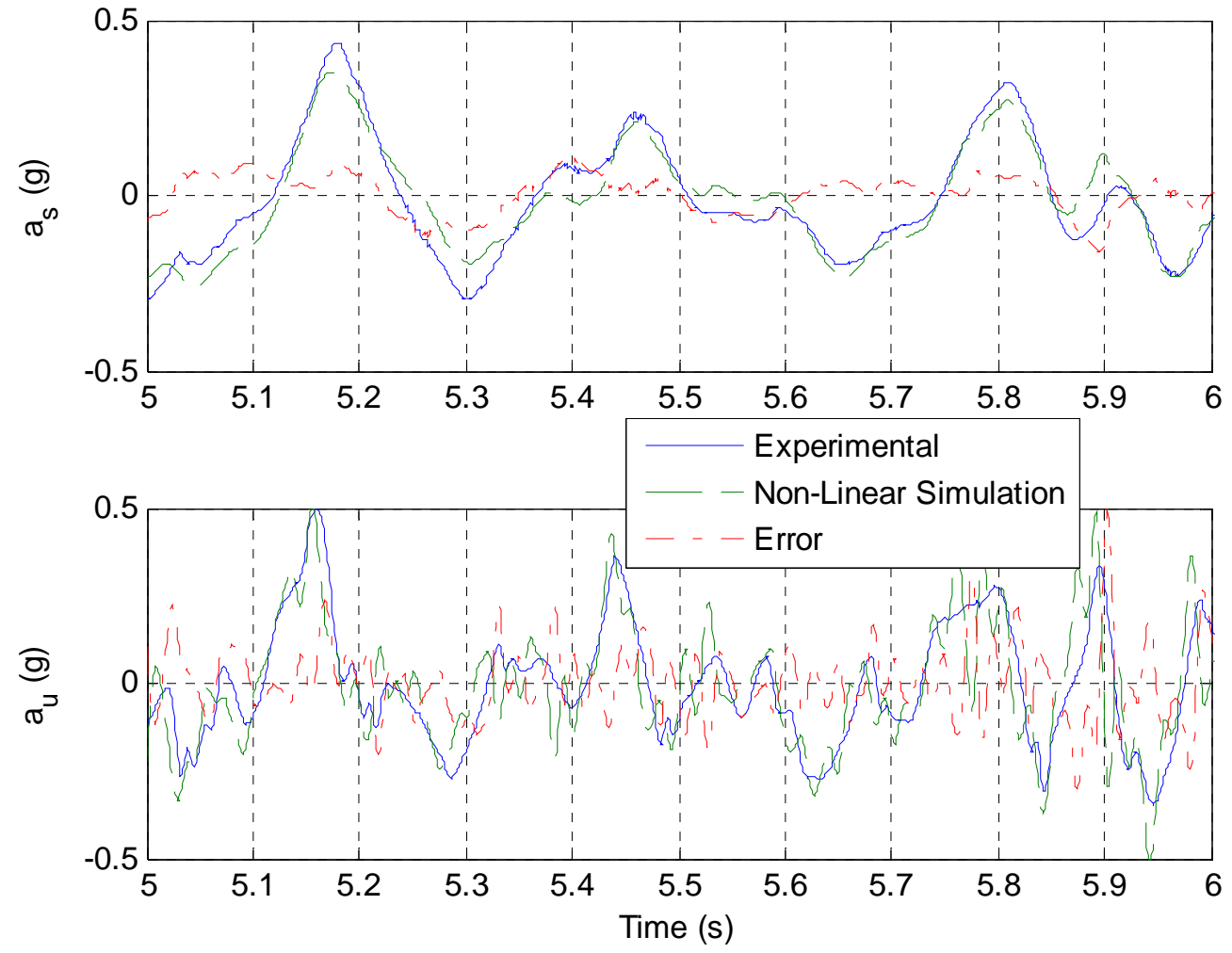

Figure 7-4 Experimental vs. Simulated Non-Linear Model Acceleration with NonOptimized Parameters 


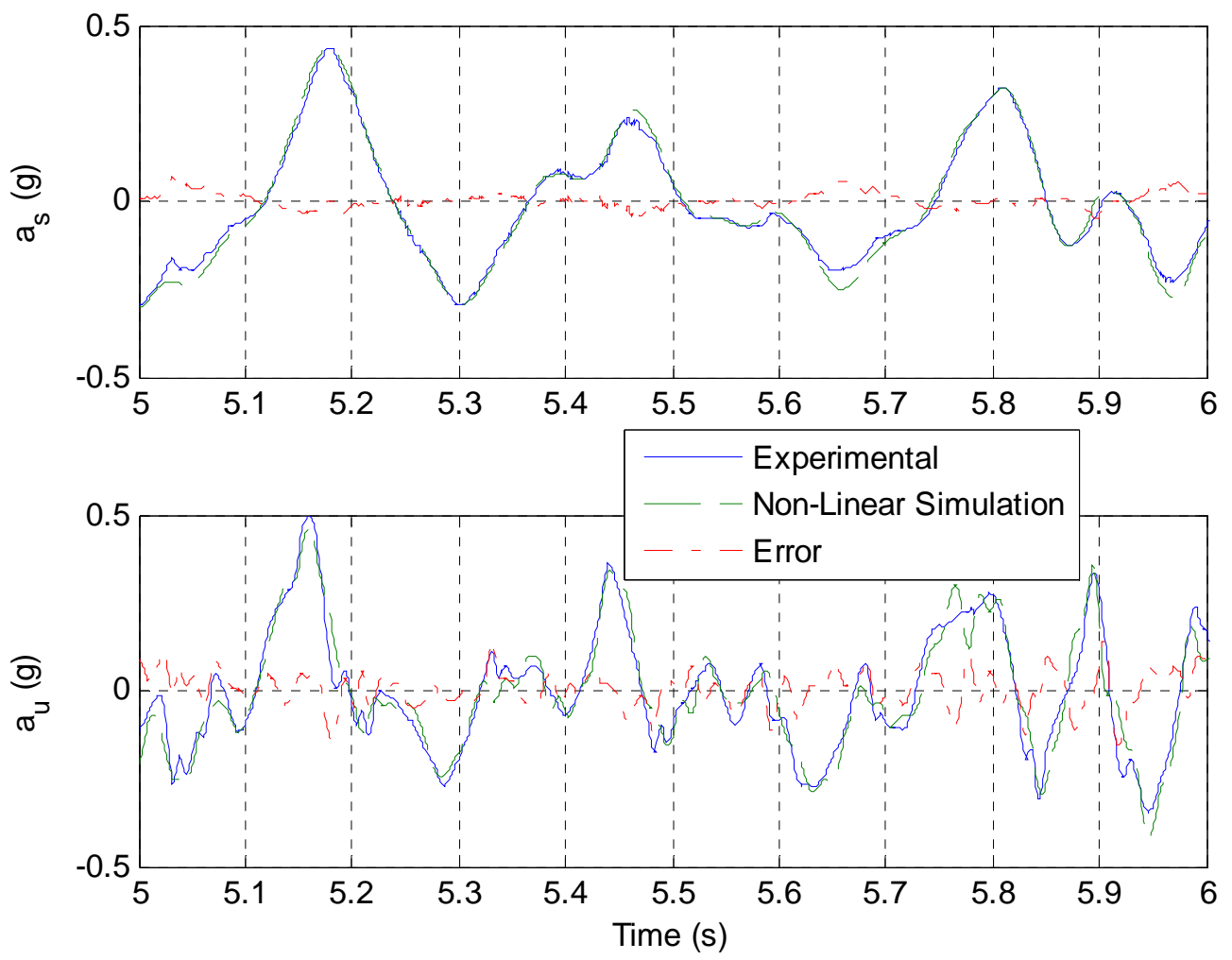

Figure 7-5 Experimental vs. Simulated Non-Linear Model Acceleration with Optimized Parameters

Table 7-1 Non-Linear Model Optimal Parameters

\begin{tabular}{|c|c|c|c|c|c|c|c|c|c|c|c|c|c|}
\hline & \multicolumn{3}{|c|}{ Mass (kg) } & \multicolumn{3}{|c|}{ Inertia $\left(\mathrm{kg}^{*} \mathrm{~m} 2\right)$} & \multicolumn{2}{|c|}{ Spring (N/m) } & \multicolumn{2}{|c|}{ Damper $\{\mathrm{N} /(\mathrm{m} / \mathrm{s})\}$} & \multicolumn{2}{|c|}{ Free Length (m) } & \multirow{2}{*}{$\begin{array}{l}\text { Moment } \\
\text { Tire }(\mathrm{m})\end{array}$} \\
\hline & $\mathbf{S}$ & C & $U$ & $\mathbf{S}$ & $\mathrm{C}$ & $U$ & Primary & Tire & Primary & Tire & Primary & Tire & \\
\hline & 127 & 162 & 63 & 17.9 & 0.04 & 0.99 & 140097 & 400024 & 3844.41 & 8013.4 & 0.57963 & 0.2692 & -0.21211 \\
\hline
\end{tabular}

\subsection{Discussion}

The non-linear model exhibits slightly better acceleration matching than the linear model. The optimized linear model produces a sprung mass performance ratio of $-18.257 \mathrm{~dB}$ while the non-linear model produces a sprung mass performance ratio of $-18.447 \mathrm{~dB}$. The optimized linear model produces an unsprung mass performance ratio of $-8.22 \mathrm{~dB}$ while the non-linear model produces an unsprung mass performance ratio of $-8.297 \mathrm{~dB}$. Insight into this result can be gained by analyzing the degree of non-linearity present in the experimental suspension. 
One measure of McPherson strut suspension non-linearity is the magnitude of strut angle change it experiences through its travel. The greater the angle change, the greater the non-linearity. Using optimized parameters, the non-linear simulation predicts a strut angle change of $\sim 0.4$ degrees; from 100.9 - 101.3 degrees. Due to its minimal strut angle change, this McPherson strut suspension can be considered a minimally non-linear system. When simulating a minimally non-linear system it is reasonable to expect a linear model of this system to perform nearly as well as a non-linear model.

The experimental suspension is from a Grand American series race car. Cars that compete in this race series attempt to maximize their performance. From a performance standpoint, it is desirable to have minimal camber change. This goal is achieved by limiting the strut angle change.

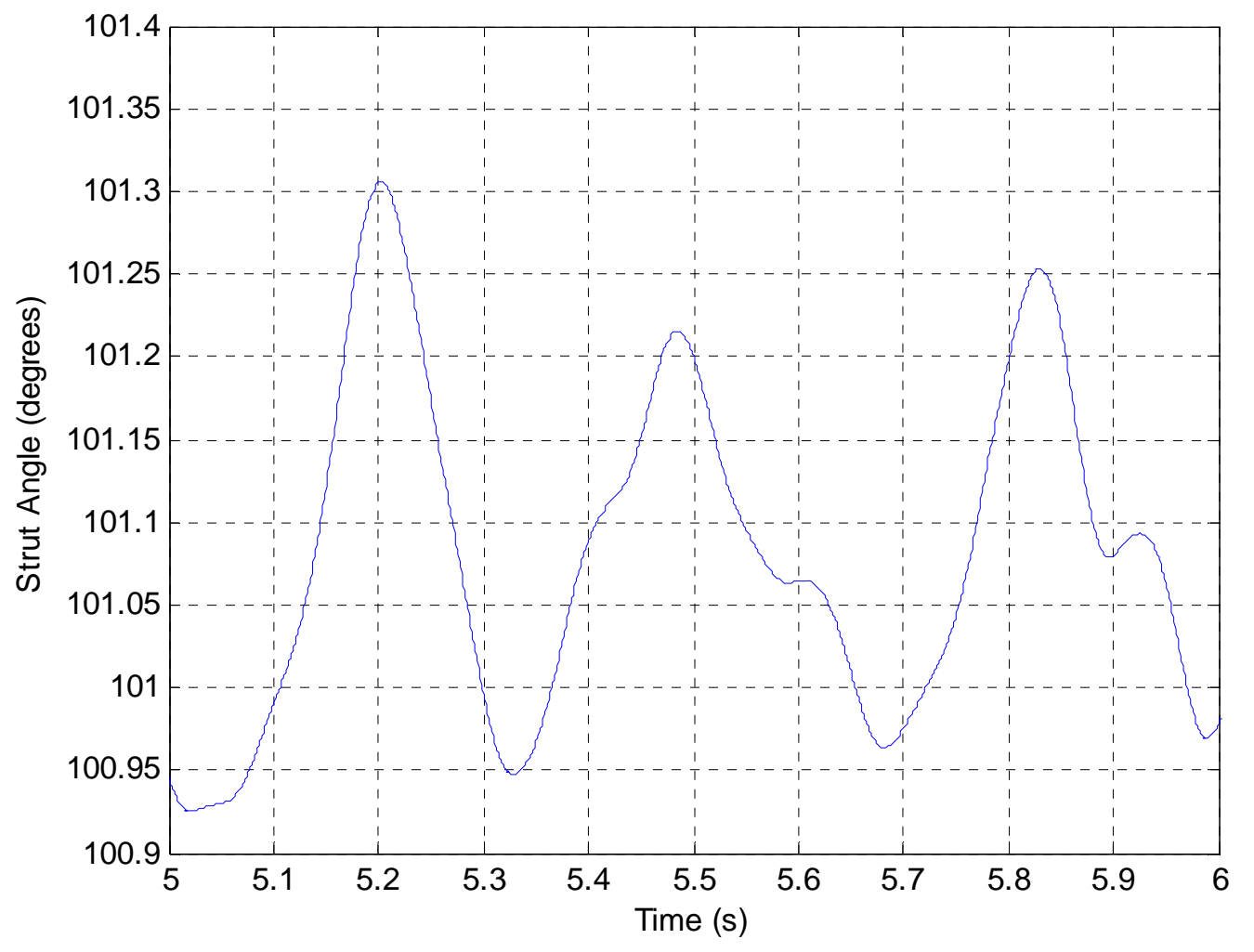

Figure 7-6 Simulated Strut Arm Angle (degrees) 


\section{LINEAR BEARING LOADS}

\subsection{Introduction}

This chapter estimates the dynamic loads applied to the quarter-car rig's sprung mass plate and linear bearings. Lagrange multipliers calculated by the non-linear multibody dynamics DAE simulation provide the means to estimate such dynamic loads.

\subsection{Joint Reaction Forces}

This section describes the theory used to obtain joint reaction forces. Motion enforced by kinematic joint constraints can also be enforced by reaction forces acting on the bodies that the joint connects (Haug [7]). For example, Figure 8-1 shows how a translational joint constraint can be represented by a constraint torque and force acting at its CG. Lagrange multipliers can be used to obtain such joint reaction forces.

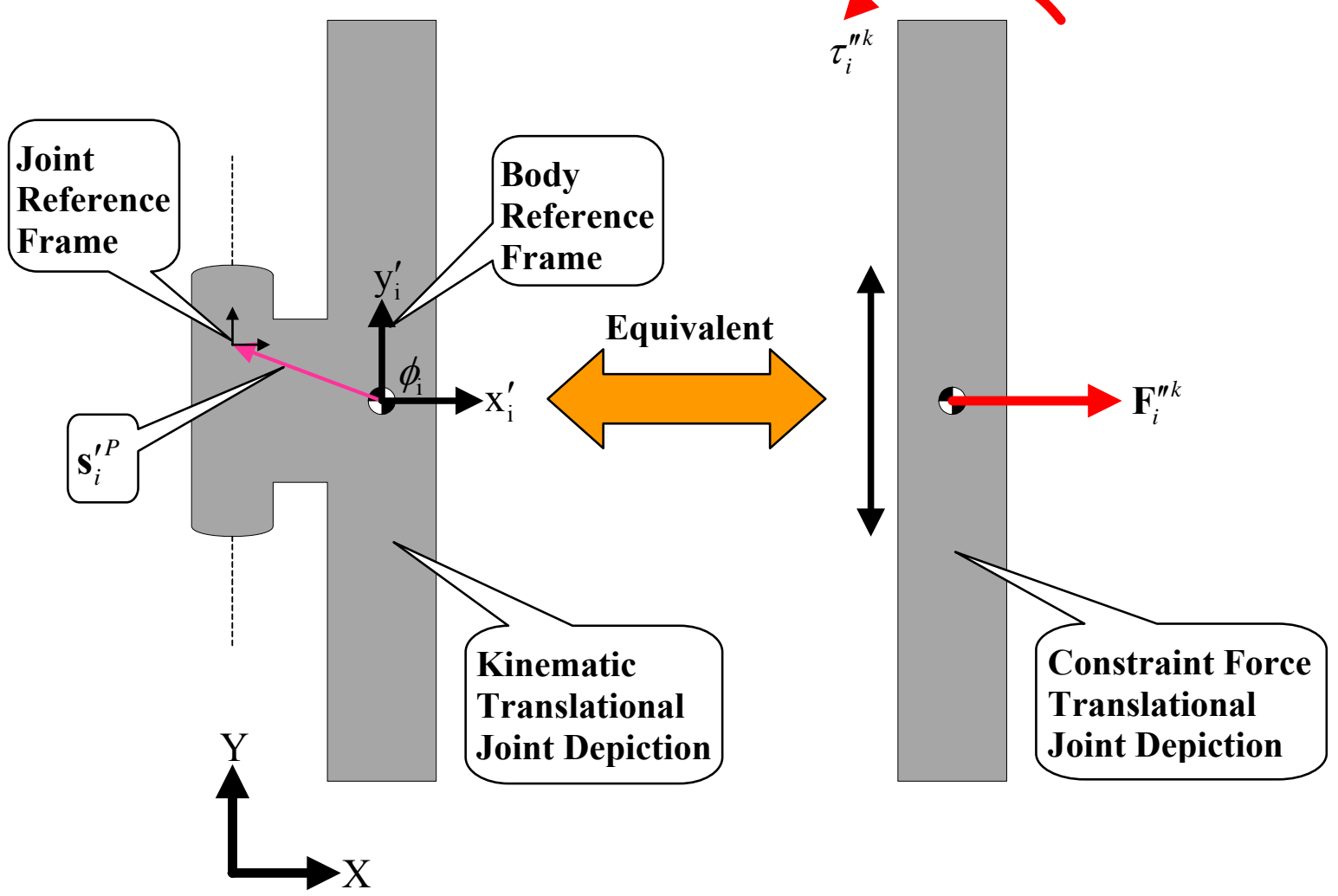

Figure 8-1 Equivalent Translational Joint Depictions 
Haug [7] provides equations for determining joint reaction force and torque from Lagrange multipliers:

$$
\begin{aligned}
& \mathbf{F}_{i}^{\prime \prime k}=-\mathbf{C}_{i}^{T} \mathbf{A}_{i}^{T} \boldsymbol{\Phi}_{\mathrm{r}_{\mathrm{i}}}^{k T} \lambda^{k} \\
& \tau_{i}^{\prime \prime k}=\left(\mathbf{s}_{i}^{\prime P T} \mathbf{B}_{i}^{T} \boldsymbol{\Phi}_{\mathrm{r}_{i}}^{k T}-\boldsymbol{\Phi}_{\phi_{i}}^{k T}\right) \lambda^{k}
\end{aligned}
$$

In these equations the superscript ' $k$ ' denotes the particular joint being analyzed. The double prime indicates that the reaction force and torque originate from the joint. The ' $\mathrm{i}$ ' subscript denotes the body to which the reaction force is being applied. The superscript ' $\mathrm{T}$ ' denotes the vector or matrix transpose. Bearing these conventions in mind, the variables used in these equations are defined as follows:

1) $\mathbf{C}_{i}$ - rotation matrix, which transforms vectors in the joint reference frame ' $\mathrm{i}$ ' to the body reference frame

2) $\mathbf{A}_{i}$ - rotation matrix, which transforms vectors in the body reference frame ' $i$ ' to the global reference frame

3) $\boldsymbol{\Phi}_{\mathrm{r}_{\mathrm{i}}}^{k}$ - partial Jacobian associated with constraint force

4) $\lambda^{k}$ - vector of Lagrange multipliers associated with the joint

5) $\mathbf{s}_{i}^{\prime P}$ - vector from body i's local reference frame to the joint reference frame

6) $\mathbf{B}_{i}$ - derivative of the rotation matrix $\mathbf{A}_{i}$ with respect to its angle

7) $\boldsymbol{\Phi}_{\phi_{1}}^{k}$ - partial Jacobian associated with constraint torque 


\subsection{Sprung Mass Force}

This section describes the process used to obtain the total sprung mass reaction force and torque. The multibody dynamics quarter-car model developed in Chapter 6 contains a translational joint connecting the sprung mass to the inertial reference frame. As seen in Figure 8-1, this translational joint constraint can be enforced by a constraint torque and force acting at the sprung mass CG. The force can be written as:

$$
\mathbf{F}_{\mathrm{S}}^{\prime \prime}=-\mathbf{C}_{\mathrm{S}}^{T} \mathbf{A}_{\mathrm{S}}^{T} \boldsymbol{\Phi}_{\mathrm{r}_{\mathrm{S}}}^{T} \boldsymbol{\lambda}
$$

To avoid confusion with the ' $\mathrm{T}$ ' superscript used to denote transpose the ' $\mathrm{t}$ ' superscript associated with a translational joint is omitted.

The joint and body reference frames will always have the same orientation with respect to the inertial axis; therefore, the angle between these frames is always zero and the rotation matrix $\mathbf{C}_{s}$ between these frames is the identity matrix:

$$
\mathbf{C}_{s}=\left[\begin{array}{cc}
\cos \phi_{k i} & -\sin \phi_{k i} \\
\sin \phi_{k i} & \cos \phi_{k i}
\end{array}\right]=\left[\begin{array}{ll}
1 & 0 \\
0 & 1
\end{array}\right]
$$

Likewise, $\mathbf{A}_{s}$ is also the identity matrix because the rotation angle between the body frame and the global frame is always zero. The Jacobian matrix with respect to this translational joint constraint is:

$$
\boldsymbol{\Phi}_{\mathrm{r}_{\mathrm{s}}}=\left[\begin{array}{ll}
0 & 0 \\
1 & 0
\end{array}\right]
$$


Thus, the reaction force is:

$$
\begin{aligned}
& \mathbf{F}_{\mathrm{S}}^{\prime \prime}=-\left[\begin{array}{ll}
1 & 0 \\
0 & 1
\end{array}\right]\left[\begin{array}{ll}
1 & 0 \\
0 & 1
\end{array}\right]\left[\begin{array}{ll}
0 & 1 \\
0 & 0
\end{array}\right]\left[\begin{array}{l}
\lambda_{1} \\
\lambda_{2}
\end{array}\right] \\
& {\left[\begin{array}{c}
\mathrm{F}_{\mathrm{Sx}}^{\prime \prime} \\
\mathrm{F}_{\mathrm{Sy}}^{\prime \prime}
\end{array}\right]=\left[\begin{array}{c}
-\lambda_{2} \\
0
\end{array}\right]}
\end{aligned}
$$

Likewise, the equation corresponding to the constraint torque can be written as:

$$
\tau_{\mathrm{S}}^{\prime \prime}=\left(\mathbf{s}_{\mathrm{S}}^{\prime P T} \mathbf{B}_{\mathrm{S}}^{T} \boldsymbol{\Phi}_{\mathrm{r}_{\mathrm{S}}}^{T}-\boldsymbol{\Phi}_{\phi_{\mathrm{S}}}^{T}\right) \boldsymbol{\lambda}
$$

In addition to the joint and body frame's being oriented at the same angle with respect to the inertial frame, they are also stacked. Thus the distance from their respective origins is zero. Accordingly, the vector from the sprung mass's local frame to the joint frame is:

$$
\mathbf{s}_{\mathrm{S}}^{\prime P T}=[\mathbf{0}]
$$

Because this vector is zero, the first term in the torque equation is zero. The Jacobian with respect to this joint's rotational constraint can be expressed as:

$$
\boldsymbol{\Phi}_{\phi_{\mathrm{S}}}=\left[\begin{array}{l}
1 \\
0
\end{array}\right]
$$

Consequently, the reaction torque is:

$$
\begin{aligned}
& \tau_{\mathrm{S}}^{\prime \prime}=\left([\mathbf{0}]-\left[\begin{array}{ll}
1 & 0
\end{array}\right]\right)\left[\begin{array}{l}
\lambda_{1} \\
\lambda_{2}
\end{array}\right] \\
& \tau_{\mathrm{S}}^{\prime \prime}=-\lambda_{1}
\end{aligned}
$$


Therefore, the Lagrange multipliers associated with the sprung mass translational constraints, $\lambda_{1}$ and $\lambda_{2}$ are the negative values of the constraint force and torque acting on the sprung mass CG:

$$
\begin{aligned}
& \mathrm{F}_{\mathrm{Sx} \rightarrow}=-\lambda_{2} \\
& \tau_{\mathrm{SU}}=-\lambda_{1}
\end{aligned}
$$

The HHT integrator solves for $\lambda_{1}$ and $\lambda_{2}$ at each time step, so the constraint force and torque acting on the sprung mass are also available at every time step. Because the intended goal is to estimate the normal loads as accurately as possible the parameters solved for in Chapter 7, parameters intended for controller design are not used to calculate normal forces. Rather, a set of parameters closer to the actual values is determined through system identification using the initial parameters in Chapter 7 but limiting the sprung mass to stay within $+-1 \%$ of its nominal value. Having the sprung mass closer to its actual value increases the accuracy of the forces and torques predicted by the Lagrange multipliers. Table $8-1$ shows the results for this system identification:

\begin{tabular}{|c|c|c|c|c|c|c|c|c|c|c|c|c|c|}
\hline & \multicolumn{3}{|c|}{ Mass (kg) } & \multicolumn{3}{|c|}{ Inertia (kg*m2) } & \multicolumn{2}{|c|}{ Spring (N/m) } & \multicolumn{2}{|c|}{ Damper $\{\mathrm{N} /(\mathrm{m} / \mathrm{s})\}$} & \multicolumn{2}{|c|}{ Free Length (m) } & \multirow{2}{*}{$\begin{array}{l}\text { Moment } \\
\text { Tire (m) }\end{array}$} \\
\hline & $\mathrm{s}$ & C & $\mathbf{U}$ & $\mathrm{s}$ & C & $U$ & Primary & Tire & Primary & Tire & Primary & Tire & \\
\hline & 238 & 5.6 & 20 & 15.7 & 0 & 0.002 & 140731 & 400048 & 3500 & 2000 & 0.65 & 0.5795 & -0.2 \\
\hline
\end{tabular}

Table 8-1 Non-Linear Model Optimized Parameters For Load Prediction

Using these parameters, the maximum force and torque in the joint obtained from system simulation between 3 and 8 seconds are:

$$
\begin{aligned}
& \operatorname{Max} \mathrm{F}_{\mathrm{Sx} \rightarrow}=93.5 \mathrm{~N} \\
& \operatorname{Max} \tau_{\mathrm{S} \cup}=-223.5 \mathrm{~N}-\mathrm{m}
\end{aligned}
$$


Likewise, the simulation estimates a minimum force and torque of:

$$
\begin{aligned}
& \operatorname{Min} \mathrm{F}_{\mathrm{Sx} \rightarrow}=-85.4 \mathrm{~N} \\
& \operatorname{Min} \tau_{\mathrm{SU}}=-539.9 \mathrm{~N}-\mathrm{m}
\end{aligned}
$$

The simulation estimates average force and torque of:

$$
\begin{aligned}
& \text { Mean } \mathrm{F}_{\mathrm{Sx} \rightarrow}=0.085 \mathrm{~N} \\
& \text { Mean } \tau_{\mathrm{SU}}=-364.12 \mathrm{~N}-\mathrm{m}
\end{aligned}
$$

Figure 8-2 presents the dynamic reaction force and torque acting on the sprung mass for a representative time period.
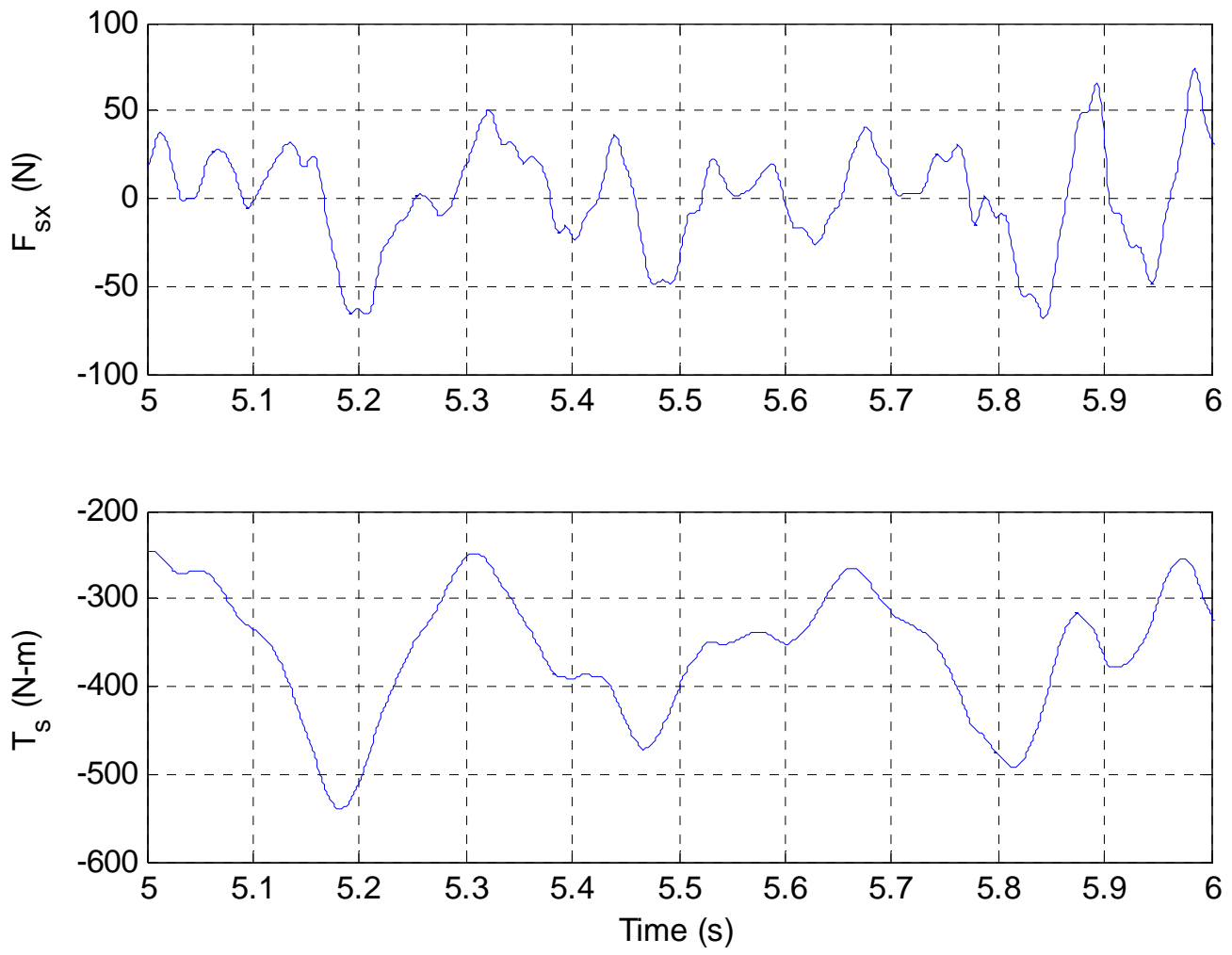

Figure 8-2 Sprung Mass Normal Force and Torque 


\subsection{Bearing Force}

This section estimates how the total sprung mass normal force is balanced between the upper and lower quarter-car rig bearings. Figure 8-3 shows the simplified quarter-car bearing constraint model used to approximate the total translational joint reaction force and torque constraining the sprung mass. In this simplified model, the two upper bearings are assumed to support equal load and are combined into one support force. The two lower bearings are similarly simplified. The bearings are assumed to have point contact roller support reaction forces. The upper and lower reaction forces $F_{B U}$ and $F_{B L}$ are distances $l_{u}=0.4912(\mathrm{~m})$ and $l_{l}=0.1184(\mathrm{~m})$ from the sprung mass CG. With this in mind, the simplified quarter-car bearing reactions forces can be equivocated with the total sprung mass translational joint reaction force and torque as follows:

Bearing Reaction Forces $=$ Total Translational Joint Reaction Force

$$
\begin{aligned}
& \mathrm{F}_{\mathrm{BU}}+\mathrm{F}_{\mathrm{BL}}=\mathrm{F}_{\mathrm{Sx} \rightarrow} \\
& \mathrm{F}_{\mathrm{BU}}+\mathrm{F}_{\mathrm{BL}}=-\lambda_{2}
\end{aligned}
$$

Bearing Reaction Torque $=$ Total Translational Joint Reaction Torque

$$
\begin{aligned}
& \sum \tau_{\mathrm{CGU}}=\tau_{\mathrm{SU}} \\
& -\mathrm{F}_{\mathrm{BU}} l_{u}+\mathrm{F}_{\mathrm{BL}} l_{l}=-\lambda_{1}
\end{aligned}
$$

Solving for the upper and lower bearing forces, we find:

$$
\begin{gathered}
\mathrm{F}_{\mathrm{BU}}=-\lambda_{2}-\frac{-\lambda_{1}-\lambda_{2} l_{u}}{\left(l_{u}+l_{l}\right)} \\
\mathrm{F}_{\mathrm{BL}}=\frac{-\lambda_{1}-\lambda_{2} l_{u}}{\left(l_{u}+l_{l}\right)}
\end{gathered}
$$

Figure 8-4 shows the estimated upper and lower bearing reaction forces. 


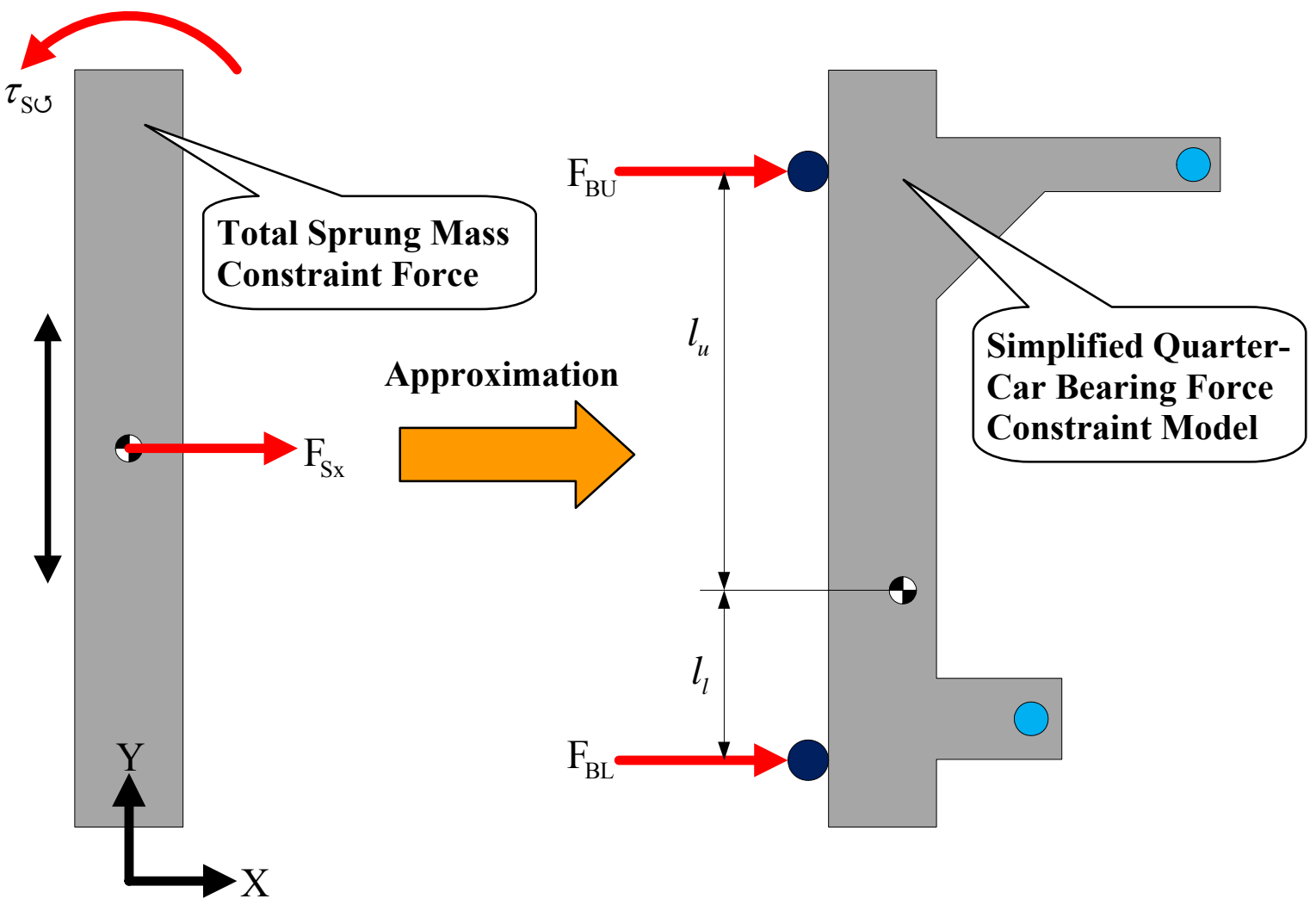

Figure 8-3 Simplified Quarter-car Bearing Reactions
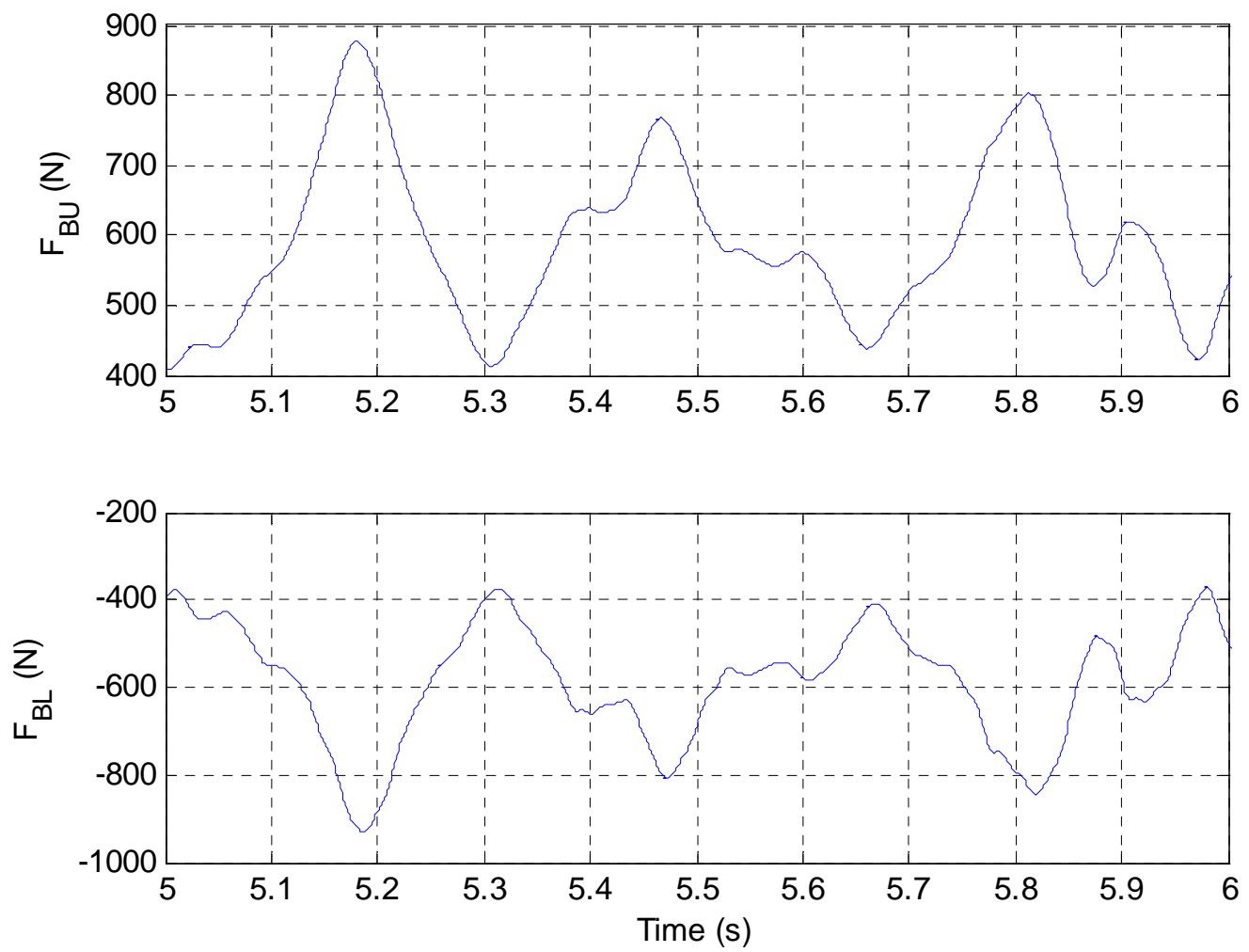

Figure 8-4 Upper and Lower Quarter-car Bearing Forces 
In the context of this thesis, the purpose for obtaining these results is to ensure that the normal loads applied to the quarter-car rig do not exceed its bearing or structural load capacity. Before using these results for that purpose, it is advisable to equip the bearings of the quarter-car rig with either strain gauges or load cells to verify that the simulated load matches the experimental load. After verification, the simulated reaction forces can be used with confidence to estimate dynamic normal loads.

Appendix B shows bearing load results with a simplified joint formulation. 


\section{CONCLUSIONS AND RECOMMENDATIONS}

This chapter summarizes the results obtained and discusses these results in the context of the thesis objectives.

\subsection{Modeling}

The first objective was to formulate linear and non-linear multibody dynamic McPherson strut suspension models and determine which is more accurate in pursuit of producing tools to aid controller design. This thesis developed and performed system identification on linear and non-linear McPherson strut quarter-car models. Using optimal parameters from their respective system identifications, the non-linear model slightly outperformed the linear model. Given that the experimental suspension under consideration is minimally non-linear, the two models' similarity in performance is expected.

As tools to aid controller design, there is value in both models. Each model is appropriate for various applications. The linear model may be most appropriate for use with McPherson strut suspensions that are minimally non-linear such as those found on race cars and where quick run time is desirable. The non-linear model may be most appropriate for use with McPherson strut suspensions that are moderately non-linear such as those found on the majority of small- and medium-sized passenger cars.

\subsection{Load Estimation}

The second objective was to use the non-linear multibody dynamics model to estimate the dynamic normal loads applied to the quarter-car rig's sprung mass plate and bearings. The non-linear model's ability to estimate sprung mass normal loads was explored in Chapter 8 using the translational joint reaction force formula and utilizing Lagrange multipliers produced by the DAE simulation. The load prediction feature implicit with the non-linear model can be used to estimate sprung mass normal loads on the quarter-car rig after validation is performed. Using strain gauges or load cells the experimental load 
can be measured and compared to the simulated load. After such verification the nonlinear model can be used with confidence for such load estimations.

\subsection{Model Template}

The third objective was to clearly describe the construction of the multibody dynamics non-linear model so that it can be used as a template for future use. The goal was to present the mathematical model in such a way that it could be modified for use with other McPherson strut configurations. Chapter 6 presents a clear description of how the nonlinear model was developed. Extension of this model to other McPherson strut suspensions would involve modifying terms of the DAE template provided in Chapter 6.

\subsection{Conclusion}

This research was successful as the objectives outlined have been met. This research aids in controller design by providing a mathematical model for McPherson strut suspensions, and aids in experimental efforts by providing a means to estimate the quarter-car rig's sprung mass dynamic normal force. This research also is useful for other McPherson strut suspension experimentation as well as for experimentation with other suspension configurations because the model construction is clearly described. It is this author's hope that these models will provide functional building blocks for controller design, future experiments, and general simulation purposes.

\subsection{Recommendations for Future Research}

Avenues for future research include continued modeling and simulation of the non-linear model, sprung mass linear bearing load verification, and use of the models for various applications.

Modeling can be continued in several ways. The primary suspension spring used in the experimental suspension is actually two springs in series. Modeling this two-element 
spring as a dual rate spring may improve accuracy. Also, the non-linear kinematic model was derived as a 2D model because its motions were assumed to be largely planar. However, a 3D model would account for any caster change or bump steer dynamics, and therefore has potential to increase the accuracy of this model. However, because the unsprung mass performance in decibels was approximately half of that achieved by the sprung mass performance, using a more comprehensive tire model may provide the most performance gain. More complete tire models, such as the model written about by Gipser [21], account for various non-linearities exhibited by tires. They also can be formulated to account for the lateral stiffness of a tire. Representing the tire with such a model has the potential to increase performance.

Simulation can be continued by choosing joint and CG locations as optimization parameters. Performance gains may be realized by including these parameters in the system identification process. By applying these adjustments, the non-linear model's accuracy may be improved. However, the benefit of such improvements must be weighed with any addition to complexity.

In addition to simulation and modeling work the sprung mass linear bearing normal force predicted by the non-linear model can be verified. By attaching either strain gauges or load cells inline with the normal load experienced by the linear bearings the normal force can be experimentally obtained. These results can be compared to the simulated results in an effort to validate the non-linear model as a sprung mass linear bearing normal force prediction tool. After verification, the model can be used with confidence for this purpose.

Future research can be undertaken by using the non-linear model for its myriad of potential applications. One such application would be to aid active and semi-active suspensions control algorithm design. Other applications include design of experiments, general dynamic simulation, and dynamic response prediction. The range of potential applications is extensive. 


\section{REFERENCES}

[1] T. D. Gillespie, Fundamentals of Vehicle Dynamics: Engineers Inc., 1992.

[2] T. Gilles, Automotive Chassis Brakes Steering \& Suspension: Thomson Delmar Learning, 2004.

[3] D. A. Mantaras, L. Laque, and C. Vera, "Development and validation of a threedimensional kinematic model for the McPherson steering and suspension mechanisms," Mechanism and Machine Theory, vol. 39, pp. 603-619, June, 2004 2004

[4] J. Daniels, Car Suspensions at Work: Theory \& Practice of Steering, Handling, \& Roadholding: Motor Racing Publications, 1988.

[5] W. F. Milliken and D. L. Milliken, Race Car Vehicle Dynamics: Society of Automotive Engineers Inc., 1995.

[6] J. Garcia de Jalon and E. Bayo, Kinematic and Dynamic Simulation of Multibody systems -The Real-Time Challenge-: Springer, 1993.

[7] E. J. Haug, Computer-Aided Kinematics and Dynamics of Mechanical System volume 1: Basic methods: Allyn and Bacon, 1989.

[8] H. Rahnejat, Multi-Body Dynamics vehicles, Machines, and Mechanisms: Professional Engineering Publishing Limited, 1998.

[9] D. Inman, Engineering Vibration Second Edition: Prentice Hall, 2000.

[10] F. D. Goncalves, "Dynamic Analysis of Semi-Active Control Techniques for Vehicle Applications," in Mechanical Engineering. vol. M.S. Blacksburg: Virginia Polytechnic Institute and State University, 2001.

[11] B. Owren and H. Simonsen, "Alternative integration methods for problems in structural dynamics," Computer Methods in Applied Mechanics and Engineering, vol. 122, pp. 1 - 10, 1995.

[12] O. J. Laurent, "Structure Preservation for Constrained Dynamics with Super Partitioned Additive Runge--Kutta Methods," SIAM Journal on Scientific Computing vol. 20, pp. 416 - 446, 19981998.

[13] D. Negrut, G. Ottarson, R. Rampalli, and A. Sajdak, "On an Implementation of the Hilber-Hughes-Taylor Method in the Context of Index 3 DifferentialAlgebraic Equations of Multibody," Journal of Computational and Nonlinear Dynamics, vol. 2, pp. $73-85,2007$.

[14] A. Cardona and M. Geradin, "Time Integration of the Equation of Motion in Mechanical Analysis," Computer and Structures, vol. 33, pp. 801 - 820, 1989.

[15] A. Cardona and M. Geradin, "Numerical Integration of Second Order Differential-Algebraic Systems in Flexible Mechanics Dynamics," in ComputerAided Analysis of Rigid and Flexible Mechanical Systems NATO ASI Series E: Applied Sciences, Edited by Pereira, Manuel F.O. Seabra and Ambrosio, Jorge. vol. 268 Kluwer Academic Publishers, 1994, pp. 501-529.

[16] R. L. Burden and J. D. Fares, Numerical Analysis 8th Edition: Thomas Brooks/Cole, 2005.

[17] J. D. Trom, J. L. Lopez, and M. J. Vanderpeg, "Modeling of a Mid-size Passenger Car Using a Multibody Dynamics Program," Journal of Mechanisms Transmissions, and Automation in Design, vol. 109, pp. 518-523, 1987. 
[18] J. Park, D. A. Guenther, and G. J. Heydinger, "Kinematic Suspension Model Applicable to Dynamic Full Vehicle Simulation," SAE Technical Paper Series No 2003-01-0859, 2003.

[19] M. K. Salaani, G. J. Heydinger, and P. A. Grygier, "Parameter Determination and Vehicle Dynamics Modeling For the NADS of the 1998 Chevrolet Malibu For the National Advanced Driving Simulator," SAE Technical Paper Series No 2001-010140, 2001.

[20] B. Ozdalyan, M. V. Blundell, and B. Phillips, "Comparison of suspension rig measurements with computer simulation," in Simulation '98. International Conference, 1998, pp. 133 - 139.

[21] M. Gipser, "FTire: a physically based application-oriented tyre model for use with detailed MBS and finite-element suspension models," Vehicle System Dynamics, vol. 43, pp. 76-91, 2005. 


\section{Appendix A - Control Arm and Unsprung Mass Moments of Inertia}

To find the moments of inertia of the control arm and unsprung mass the following procedure was used:

1) Sketch the composite body and place its origin with its $X-Y$ axis. Include sketches of each component with their respective CG locations and distances from the component $\mathrm{CG}$ to the composite body origin.

2) Calculate the position of the composite body's CG from the following equations:

$$
\begin{gathered}
\bar{x}=\frac{\sum m_{i} x_{i}}{\sum m_{i}} \\
\bar{y}=\frac{\sum m_{i} y_{i}}{\sum m_{i}}
\end{gathered}
$$

where $\bar{x}$ and $\bar{y}$ are the distances from the composite body origin to the composite body CG, $m_{i}$ is the mass of component $\mathrm{i}$, and $x_{i}$ and $y_{i}$ are the distances from component i's CG to the composite body origin.

3) Use the moment of inertia equation for a uniformly dense rectangular section to calculate each component's moment of inertia:

$$
j_{i}^{\prime}=\frac{1}{12} m_{i}\left(a_{i}^{2}+b_{i}^{2}\right)
$$

where $j_{i}^{\prime}$ is the moment of inertia of component $i$ about its $\mathrm{CG}$, and $a_{i}$ and $b_{i}$ are the length and width of the rectangle used to approximate component $i$.

4) Use the parallel axis theorem to determine the moment of inertia of each component about the composite CG: 


$$
J_{i}^{*}=J_{i}+m_{i} \rho_{i}^{2}
$$

where $J_{i}^{*}$ is component i's moment of inertia about the composite CG, $J_{i}$ is component i's inertia about its own CG, and $\rho_{i}^{2}$ is the squared distance from component i's CG to the composite body CG.

5) Add $J_{i}^{*}$ for components that add to the composite body shape and subtract $J_{i}^{*}$ for components that subtract from the composite body shape to find the total composite body moment of inertia.

\section{Control Arm}

The two-piece control arm and tie rod present on the experimental quarter-car rig was modeled kinematically as a single link and is termed here as the composite control arm. Because of this kinematic simplification, its moment of inertia is modeled as the composite of the two-piece control arm and tie rod links. Shown in Figure A-1 is a sketch of the composite control arm and the components that it consists of. The global axis is located along the left edge of the tie rod. 


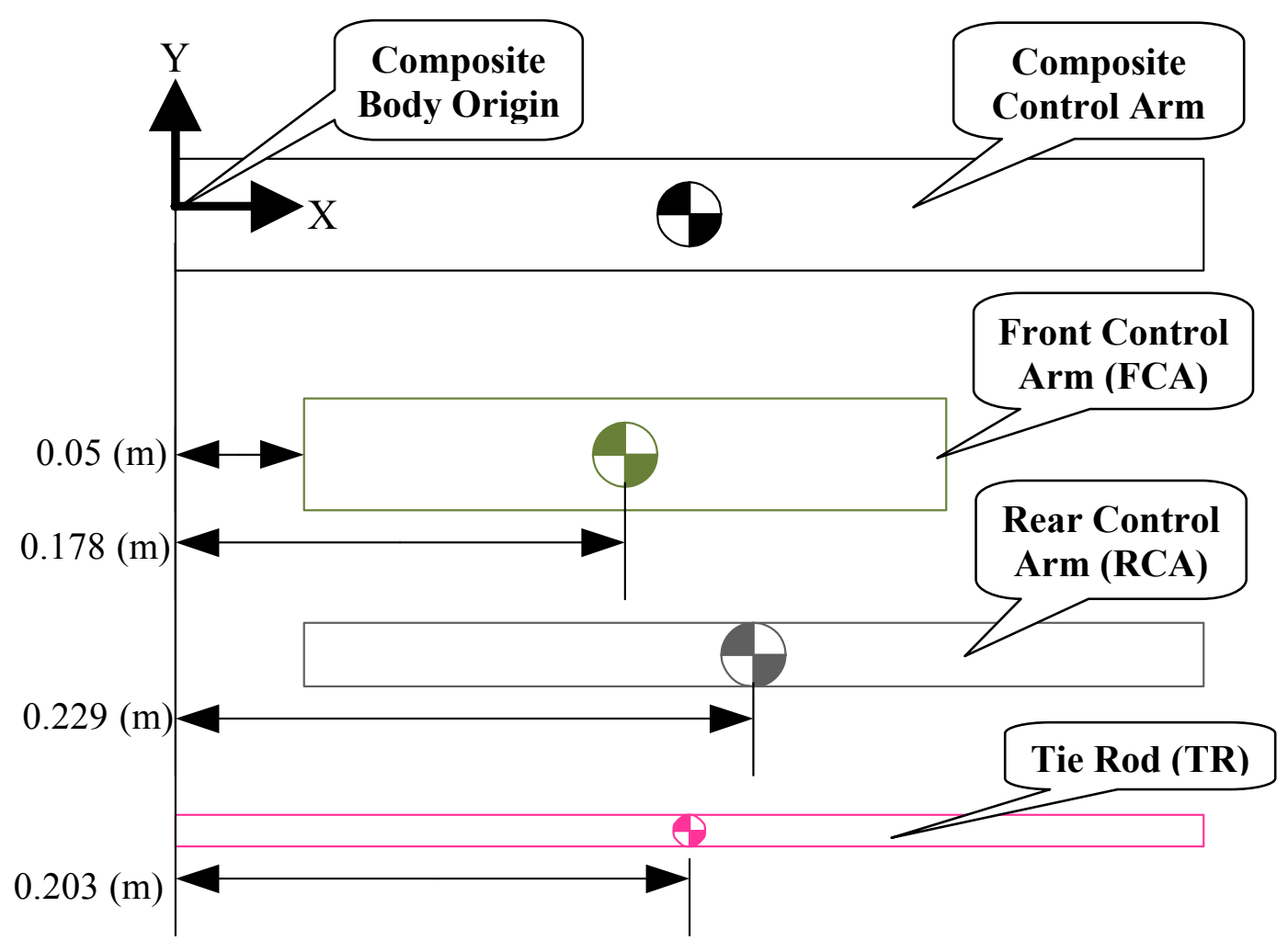

Figure A-1 Composite Control Arm and Components

The Y-direction CG position of each control arm component is located at its respective center. Thus the $\mathrm{Y}$ position of the composite control arm $\mathrm{CG}$ is also at its respective center. Bearing this in mind, only the $\mathrm{X}$ position of the composite control arm's $\mathrm{CG}$ needed to be solved. Table A-1 uses the equation A.1 to calculate the control arm composite CG X position:

Table A-1 Control Arm Composite Body CG X Position

\begin{tabular}{|c|c|c|c|c|}
\hline $\begin{array}{c}\text { Component } \\
\text { Body }\end{array}$ & $\begin{array}{c}\boldsymbol{m}_{\boldsymbol{i}} \\
(\mathbf{k g})\end{array}$ & $\begin{array}{c}\boldsymbol{x}_{\boldsymbol{i}} \\
(\mathbf{m})\end{array}$ & $\begin{array}{c}\boldsymbol{m}_{\boldsymbol{i}}{ }^{*} \boldsymbol{x}_{\boldsymbol{i}} \\
(\mathbf{k g}-\mathbf{m})\end{array}$ & $\begin{array}{c}\text { Composite } \\
\text { Body CG (m) }\end{array}$ \\
\hline FCA & 0.653 & 0.178 & 0.11623 & \multirow{2}{*}{ X Position } \\
\cline { 1 - 4 } RCA & 1.338 & 0.229 & 0.3064 & \multirow{2}{*}{$\mathbf{0 . 2 0 9 6}$} \\
\hline TR & 0.788 & 0.203 & 0.15996 & \\
\hline Total & 2.779 & & 0.5826 & \\
\hline
\end{tabular}


Next, the moment of inertia of each control arm component was calculated using equation A.3. Table A-2 shows the results of these calculations for the control arm components:

Table A-2 Control Arm Component Moments of Inertia

\begin{tabular}{|c|c|c|c|c|}
\hline $\begin{array}{c}\text { Component } \\
\text { Body }\end{array}$ & $\begin{array}{c}\boldsymbol{m}_{\boldsymbol{i}} \\
(\mathbf{k g})\end{array}$ & $\begin{array}{c}\text { Length } \\
(\mathbf{m})\end{array}$ & $\begin{array}{c}\text { Width } \\
(\mathbf{m})\end{array}$ & $\begin{array}{c}\mathbf{j}^{\prime} \\
\mathbf{( k g - m )}\end{array}$ \\
\hline FCA & 0.653 & 0.254 & 0.0445 & 0.003619 \\
\hline RCA & 1.338 & 0.356 & 0.0254 & 0.014203 \\
\hline TR & 0.788 & 0.406 & 0.0127 & 0.010835 \\
\hline
\end{tabular}

Next, the parallel axis theorem, equation A.4, was used to determine the moment of inertia of each control arm component about the composite control arm CG. Table A-3 shows the results of this computation and sums the component inertias to arrive at the composite control arm moment of inertia:

Table A-3 Control Arm Composite Body Moment of Inertia

\begin{tabular}{|c|c|c|c|c|c|}
\hline $\begin{array}{c}\text { Component } \\
\text { Body }\end{array}$ & $\begin{array}{c}\mathbf{j}^{\prime} \\
(\mathbf{k g}-\mathbf{m})\end{array}$ & $\begin{array}{c}\text { Mass } \\
(\mathbf{k g})\end{array}$ & $\begin{array}{c}\text { Composite Body } \\
\text { CG Position (m) }\end{array}$ & $\begin{array}{c}\text { Local CG } \\
\text { Position (m) }\end{array}$ & $\begin{array}{c}\mathbf{J}_{\mathbf{i}}{ }^{*} \\
(\mathbf{k g}-\mathbf{m})\end{array}$ \\
\hline FCA & 0.00362 & 0.653 & 0.209643757 & 0.178 & 0.0043 \\
\hline RCA & 0.0142 & 1.338 & 0.209643757 & 0.229 & 0.0147 \\
\hline TR & 0.01083 & 0.788 & 0.209643757 & 0.203 & 0.0109 \\
\hline $\mathbf{J}^{*}=$ Control Arm Composite Body Moment of Inertia (kg-m) & $\mathbf{0 . 0 2 9 8}$ \\
\hline
\end{tabular}

\section{Unsprung Mass}

The unsprung mass moment of inertia was calculated with the same procedure used for the control arm. The composite unsprung mass body was approximated with three rectangles; one representing the strut, one representing the hub, and one representing the wheel assembly. Figure A-2 shows the component representation of the composite unsprung mass. The global axis is located at the base of the strut coincident with revolute joint B. 


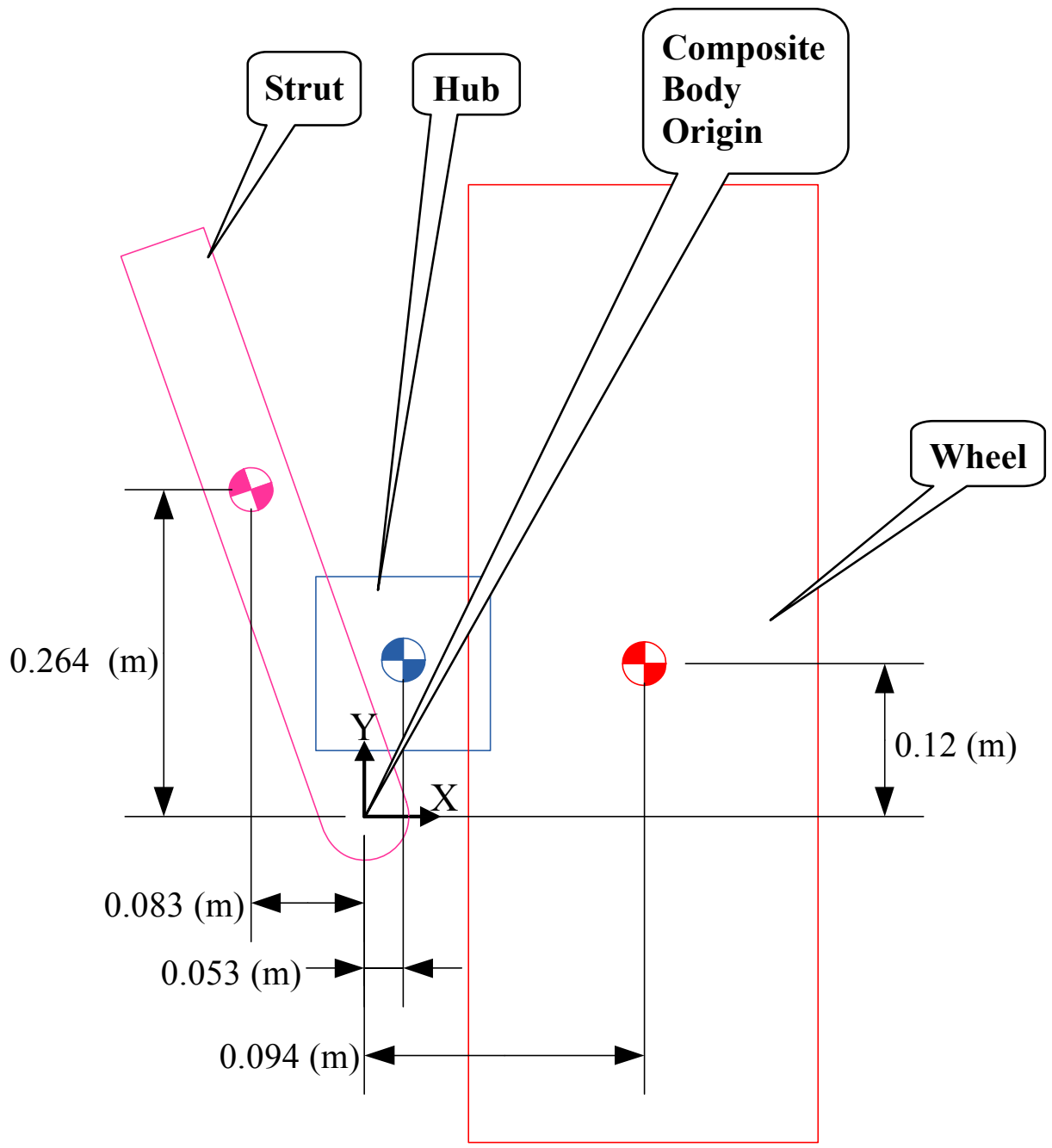

Figure A-2 Composite Unsprung Mass and Components

Table A-4 uses the equations A.1 and A.2 to calculate the composite unsprung mass CG $\mathrm{X}$ and $\mathrm{Y}$ positions:

Table A-4 Unsprung Mass Composite Body CG X-Y Position

\begin{tabular}{|c|c|c|c|c|c|c|c|}
\hline $\begin{array}{l}\text { Component } \\
\text { Body }\end{array}$ & $\begin{array}{c}m_{i} \\
(\mathrm{~kg})\end{array}$ & $\begin{array}{l}X_{i} \\
(\mathrm{~m})\end{array}$ & $\begin{array}{l}y_{i} \\
(\mathrm{~m})\end{array}$ & $\begin{array}{l}m_{i}{ }^{*} X_{i} \\
(\mathrm{~kg}-\mathrm{m})\end{array}$ & $\begin{array}{l}m_{i}{ }^{*} y_{i} \\
(\mathrm{~kg}-\mathrm{m})\end{array}$ & \multicolumn{2}{|c|}{$\begin{array}{c}\text { Composite Body } \\
\text { CG (m) }\end{array}$} \\
\hline Strut & 5.425 & -0.083 & 0.264 & -0.4503 & 1.4322 & \multirow{3}{*}{$\begin{array}{c}X \\
\text { Position }\end{array}$} & \multirow{3}{*}{$\begin{array}{c}Y \\
\text { Position }\end{array}$} \\
\hline Hub & 5.425 & 0.053 & 0.12 & 0.28753 & 0.651 & & \\
\hline Wheel & 28.97 & 0.094 & 0.12 & 2.72318 & 3.4764 & & \\
\hline Total & 39.82 & & & 2.56043 & 5.5596 & 0.0643 & 0.1396 \\
\hline
\end{tabular}


Next, the moment of inertia of each component was calculated using equation A.3. Table A-5 shows the results of these calculations for the unsprung mass components:

Table A-5 Unsprung Mass Component Moments of Inertia

\begin{tabular}{|c|c|c|c|c|}
\hline $\begin{array}{c}\text { Component } \\
\text { Body }\end{array}$ & $\begin{array}{c}\boldsymbol{m}_{\boldsymbol{i}} \\
(\mathbf{k g})\end{array}$ & $\begin{array}{c}\text { Length } \\
(\mathbf{m})\end{array}$ & $\begin{array}{c}\text { Width } \\
(\mathbf{m})\end{array}$ & $\begin{array}{c}\mathbf{j}^{\prime} \\
(\mathbf{k g}-\mathbf{m})\end{array}$ \\
\hline Strut & 5.425 & 0.553 & 0.05 & 0.139381 \\
\hline Hub & 5.425 & 0.152 & 0.181 & 0.025256 \\
\hline Wheel & 28.97 & 0.66 & 0.229 & 1.178212 \\
\hline
\end{tabular}

Next, the parallel axis theorem, equation A.4, was used to determine the moment of inertia of each unsprung mass component about the composite control arm CG. Table A-6 shows the results of this computation and sums the component inertias to arrive at the composite unsprung mass moment of inertia:

Table A-6 Unsprung Mass Composite Body Moment of Inertia

\begin{tabular}{|c|c|c|c|c|c|c|c|}
\hline \multicolumn{8}{|c|}{ Unsprung Mass Composite Body Moment of Inertia } \\
\hline $\begin{array}{l}\text { Component } \\
\text { Body }\end{array}$ & $\begin{array}{c}\mathrm{j}^{\prime} \\
(\mathrm{kg}-\mathrm{m})\end{array}$ & $\begin{array}{c}\text { Mass } \\
(\mathrm{kg})\end{array}$ & $\begin{array}{l}\text { Composite Body } \\
\text { CG X Position (m) }\end{array}$ & $\begin{array}{l}\text { Composite Body } \\
\text { CG Y Position (m) }\end{array}$ & $x_{i}(\mathrm{~m})$ & $y_{i}(\mathrm{~m})$ & $\begin{array}{c}\mathrm{J}_{\mathrm{i}}^{*} \\
(\mathrm{~kg}-\mathrm{m})\end{array}$ \\
\hline Strut & 0.13938 & 5.425 & 0.0643001 & 0.139618282 & -0.083 & 0.264 & 0.341 \\
\hline Hub & 0.02526 & 5.425 & 0.0643001 & 0.139618282 & 0.053 & 0.12 & 0.028 \\
\hline Wheel & 1.17821 & 28.97 & 0.0643001 & 0.139618282 & 0.094 & 0.12 & 1.2149 \\
\hline \multicolumn{7}{|c|}{$\mathrm{J}^{*}=$ Unsprung Mass Composite Body Moment of Inertia (kg-m) } & 1.584 \\
\hline
\end{tabular}




\section{Appendix B - Bearing Loads with Simplified Constraints}

Alternative bearing load results can be obtained by substituting the translational constraint; that is $\phi_{\mathrm{S}}=x_{\mathrm{S}}=0$ and the geometric condition of $s_{\mathrm{Cy}}^{\prime \mathrm{A}}=s_{\mathrm{Cy}}^{\prime \mathrm{B}}=s_{\mathrm{Uy}}^{\prime \mathrm{P}}=0$ into the non-linear constraint vector to obtain the following simplified constraint vector:

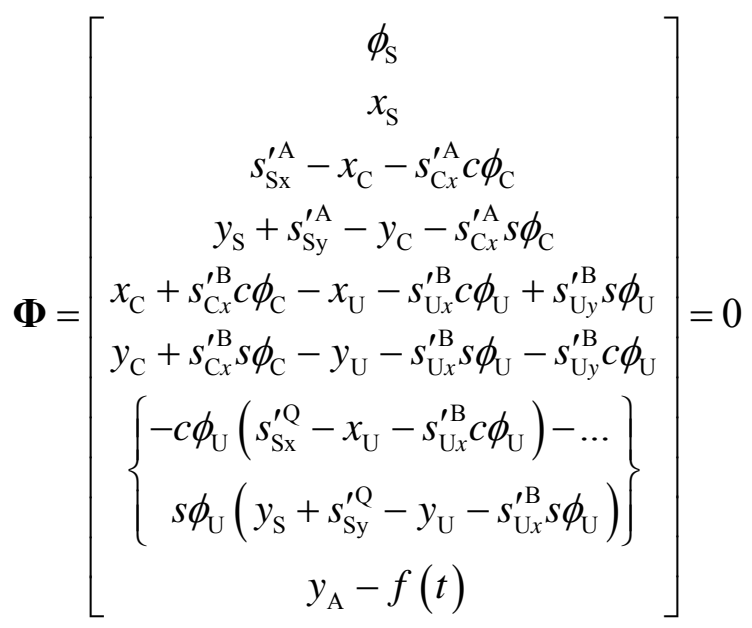

This simplification affects the Jacobian of the constraints and the acceleration equations. By keeping the translational joint constraints, the Lagrange multipliers associated with these constraints can be determined and an alternative estimation for the sprung mass reaction forces can be determined. Simulating the non-linear system with these simplified constraints and the parameters solved for in chapter 8 , the maximum reaction force and torque acting on the translational joint between simulation times 3 and 8 seconds are:

$$
\begin{aligned}
& \operatorname{Max} F_{S x \rightarrow}=219 N \\
& \operatorname{Max} \tau_{S u}=-424 N-m
\end{aligned}
$$

Likewise, the simulation estimates a minimum force and torque of:

$$
\begin{aligned}
& \text { Min } \mathrm{F}_{\mathrm{Sx} \rightarrow}=87 \mathrm{~N} \\
& \operatorname{Min} \tau_{\mathrm{SU}}=-986.5 \mathrm{~N}-\mathrm{m}
\end{aligned}
$$


The simulation estimates average force and torque of:

$$
\begin{aligned}
& \text { Mean } \mathrm{F}_{\mathrm{Sx} \rightarrow}=143.7 \mathrm{~N} \\
& \text { Mean } \tau_{\mathrm{Su}}=-673.3 \mathrm{~N}-\mathrm{m}
\end{aligned}
$$

Figure B-1 presents the dynamic reaction force and torque acting on the sprung mass for a representative time period. Figure B-2 shows the estimated upper and lower bearing reaction forces.
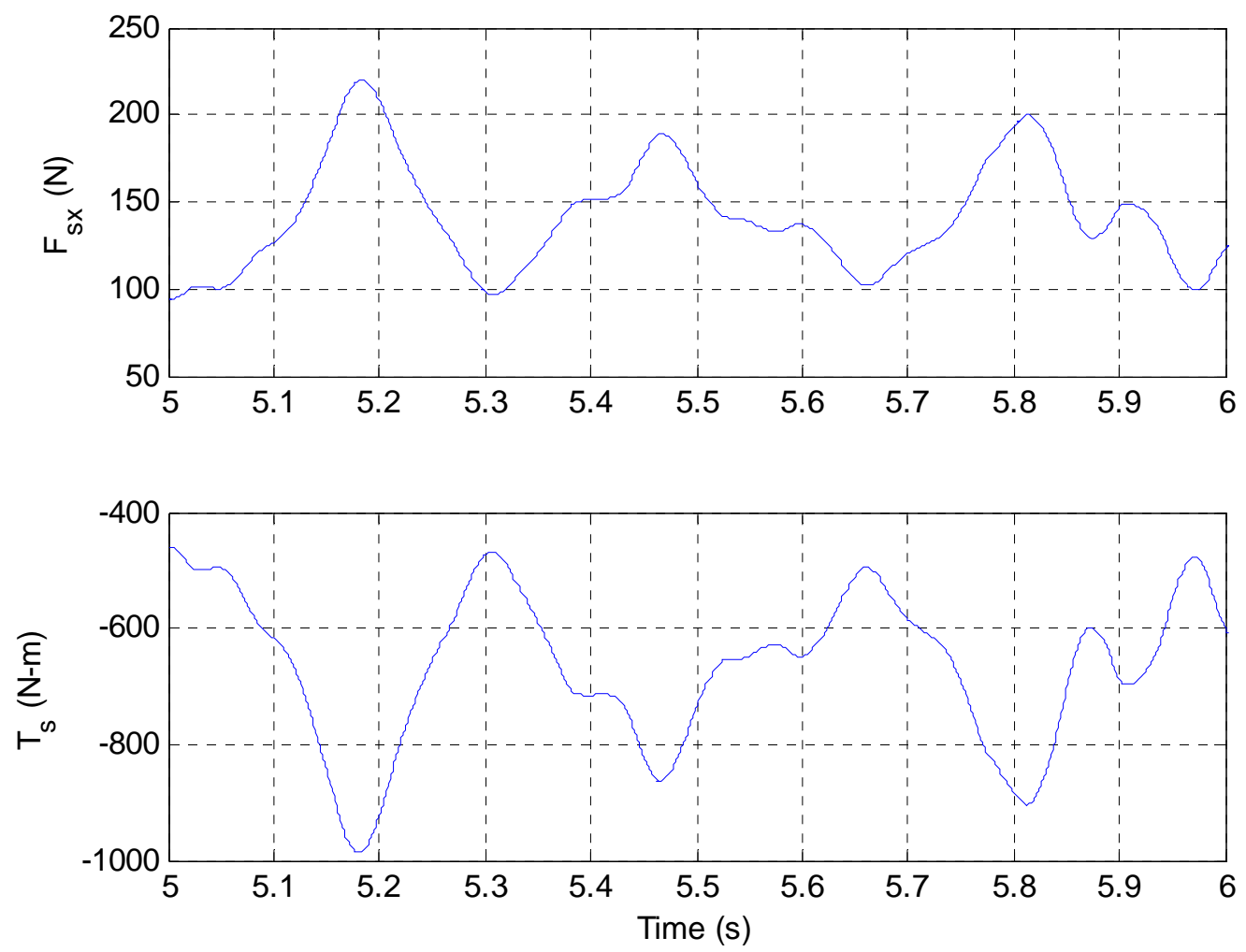

Figure B-1 Sprung Mass Normal Force and Torque from Simplified Constraints 

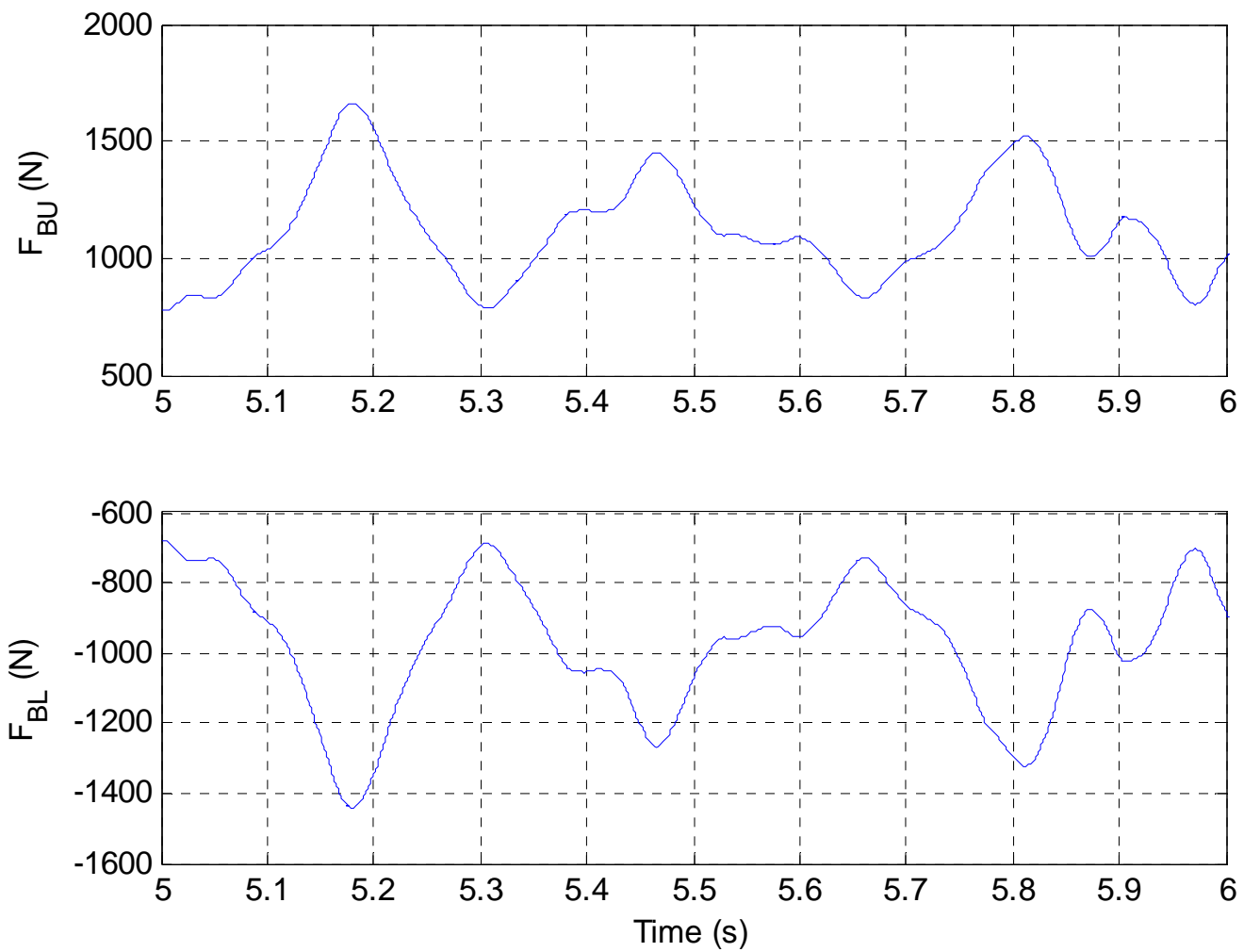

Figure B-2 Upper and Lower Quarter-car Bearing Forces from Simplified Constraints

These results were presented to show one way in which the translational joint constraint force and torque and, hence, the bearing loads can be affected by the simulation model. Other factors that will affect this result include CG placement and parameter values. Before using this model as a design tool, sufficient experience with how the simulated and experimental bearing loads correlated to one another is needed. 